\title{
THE IMPACT OF INCREASED COLLATERAL FLOW ON TRANSVERSE ARTERIOLAR MICROVASCULAR TREE MORPHOLOGY
}

\author{
A Thesis \\ Presentenced to the Faculty of \\ California Polytechnic State University, \\ San Luis Obispo
}

\author{
In Partial Fulfillment \\ Of the requirements for the Degree \\ Master of Science in Biomedical Engineering
}

By

David Danzeiser

April 2012 
(C) 2012

David Danzeiser

ALL RIGHTS RESERVED 
COMMITTEE MEMBERSHIP

TITLE:

THE IMPACT OF INCREASED COLLATERAL FLOW

ON TRANSVERSE ARTERIOLAR

MICROVASCULAR TREE MORPHOLOGY

AUTHOR: David Danzeiser

DATE SUBMITTED: April, 2012

COMMITTEE CHAIR: $\quad$ Trevor R. Cardinal, PhD.

COMMITTEE MEMBER: Lily Laiho, PhD.

COMMITTEE MEMBER: Kristen O'Halloran Cardinal, PhD. 


\begin{abstract}
The Impact of Increased Collateral Flow on Transverse Arteriolar Microvascular Tree Morphology

David Danzeiser
\end{abstract}

The goal of this study was to understand how arteriogenesis impacts transverse arteriolar tree structure. The previous process involved a vascular casting technique that utilized black India ink as the casting agent and manual measurement of vessel morphology with ImageJ. This study decreased the complexity of the casted samples by using a casting compound called Microfil. Microfil's viscosity can be adjusted and through experimentation, a viscosity was found that stopped at the capillary level, allowing only the arterial side of the circulation to be casted. A macro in ImageJ was written to help with morphological measurement by bundling vessel length and diameter into one measurement instead of two. These two adjustments were then used in a ligated mouse model to compare healthy and remodeled vascular networks. The analysis revealed that the only significant difference for length and diameter measurements was in the diameters of the collateral. This indicates that it's possible that stimulating arteriogenesis in a clinical environment should not cause any negative effects in the collateral-containing muscle. However, this observation is potentially limited by the differences between the model and patient. 


\section{Acknowledgements}

I would like to express my enormous gratitude to Dr. Trevor R. Cardinal for all of his help, guidance and patience throughout this project. I would also like to thank the other members of my thesis committee, Dr. Lily Laiho and Dr. Kristen O'Halloran Cardinal, for contributing their valuable time and suggestions. Thanks also to my fellow MATR lab members for the help and encouragement during my project.

Thank you to John, Juanita, Sean, Emma and the late Gloria for being such a great family. I will always be grateful that you have supported me not only in this thesis but also with any of my endeavors. The constant encouragement always provides the motivation to continue moving forward, always learning, always improving.

To my friends: thank you for being a part of my life and for all the joy and good times you have provided me.

"A master in the art of living draws no sharp distinction between his work and his play; his labor and his leisure; his mind and his body; his education and his recreation. He hardly knows which is which. He simply pursues his vision of excellence through whatever he is doing, and leaves others to determine whether he is working or playing. To himself, he always appears to be doing both." Lawrence Pearsall Jacks 


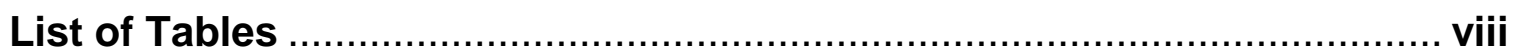

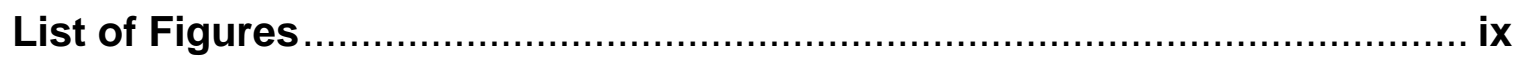

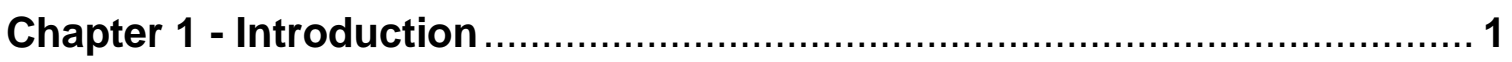

Normal Function of Circulatory System .................................................... 1

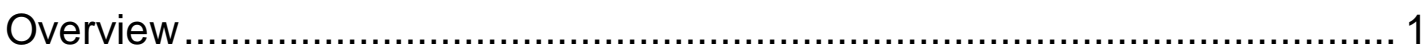

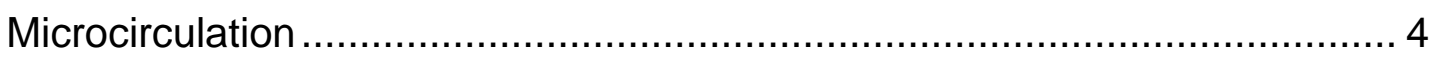

Dysfunction in the Circulatory System ..................................................... 5

Peripheral Artery Disease …............................................................... 5

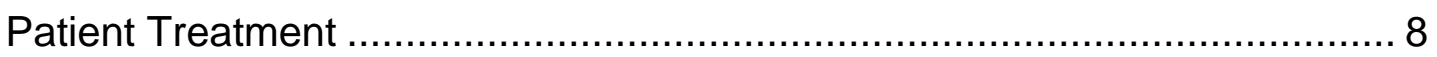

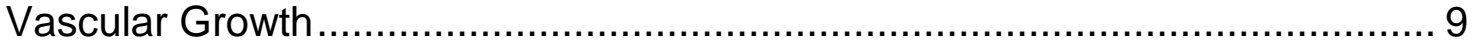

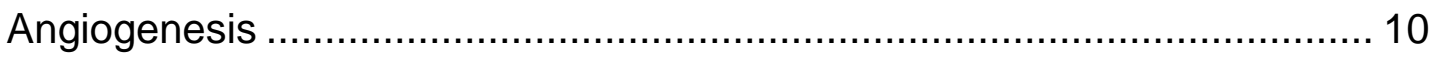

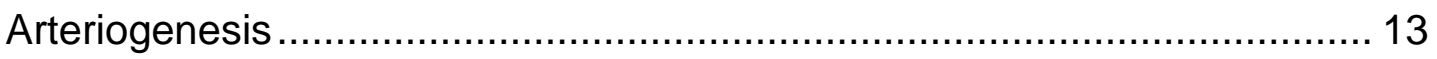

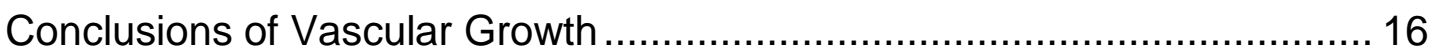

Attempts to Stimulate and Assess Vascular Growth ................................. 17

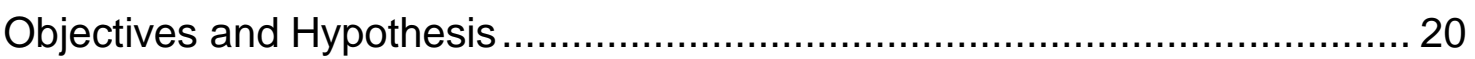

Chapter 2 - Development of an Arterial-Specific Vascular Casting

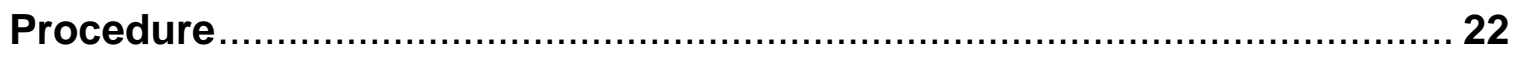

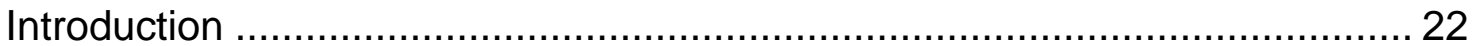

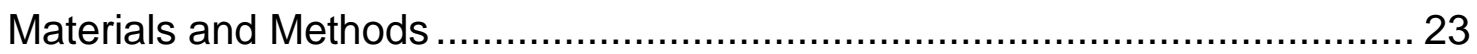

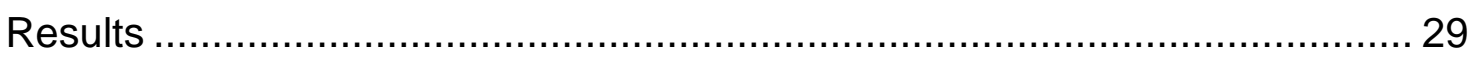

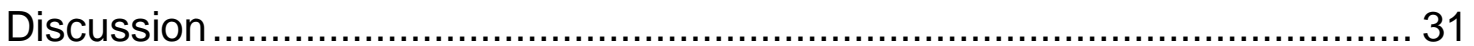

Chapter 3 - Development of an Image Morphometry Macro …................. 37

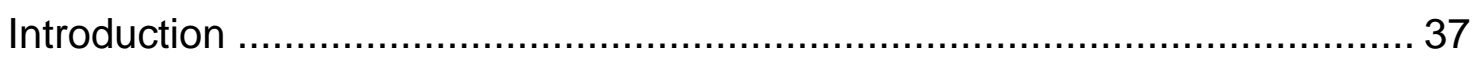

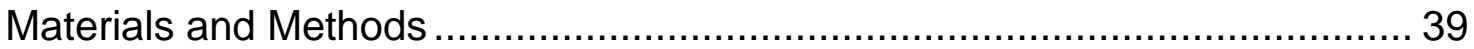

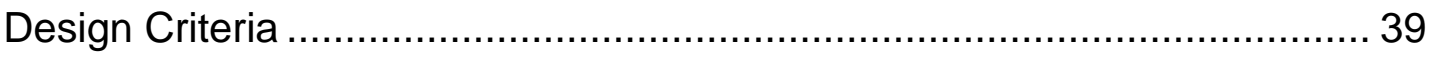

Vessel Morphometry Procedure ............................................................ 40

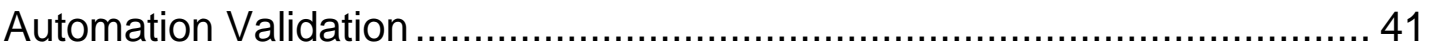

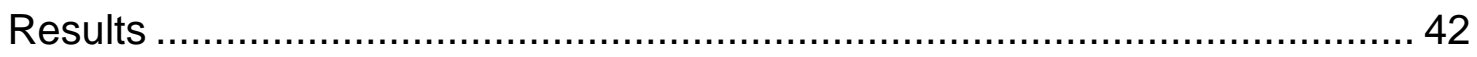

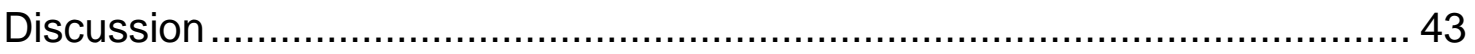




\section{Chapter 4 - The Impact of Collateral Enlargement on Transverse Arteriolar}

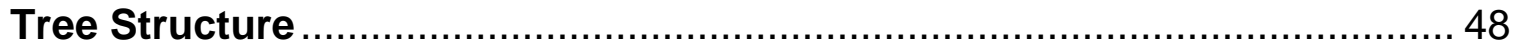

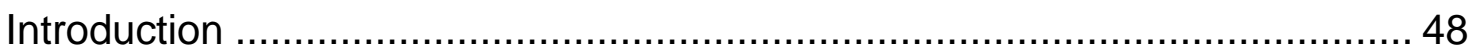

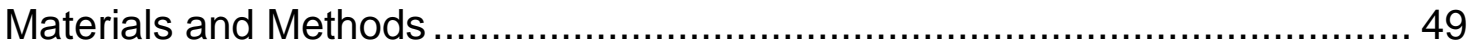

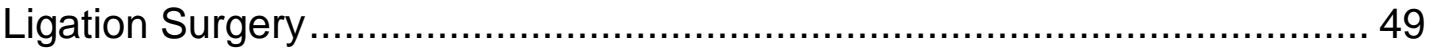

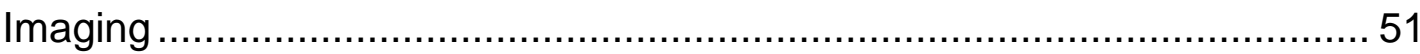

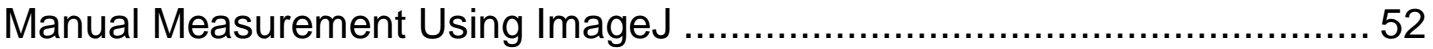

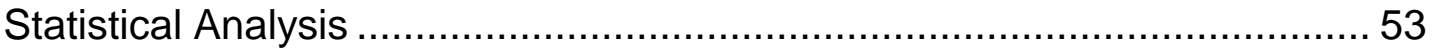

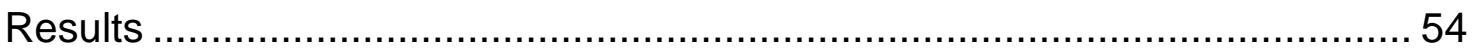

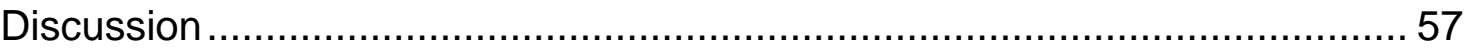

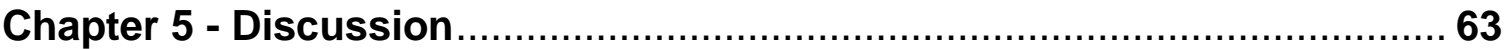

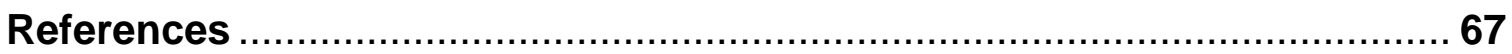

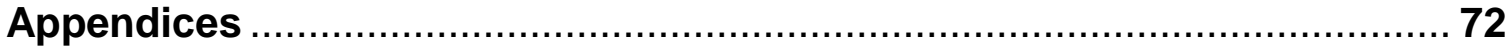

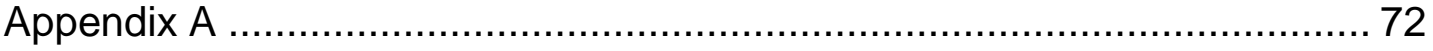

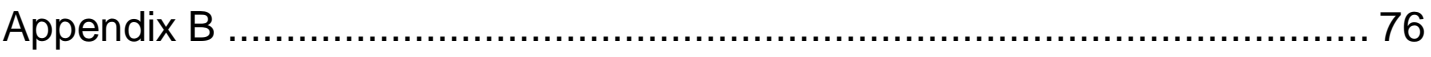

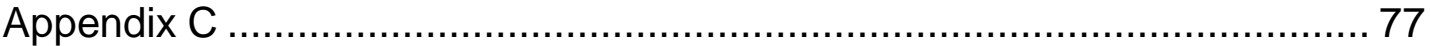

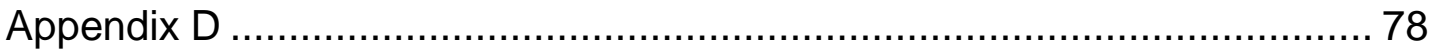

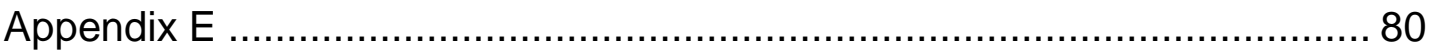

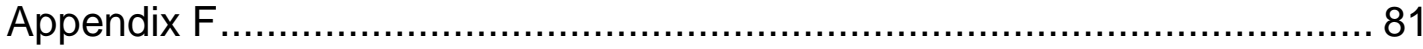

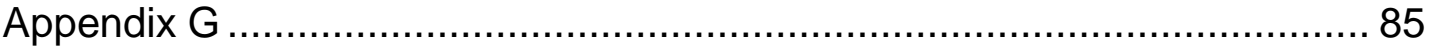

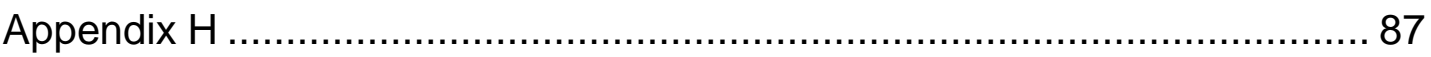

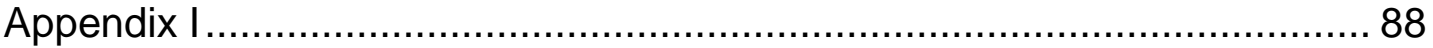

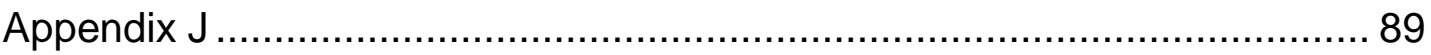

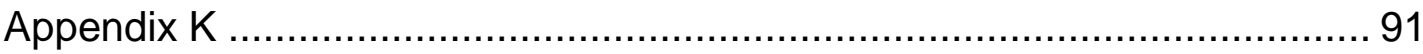

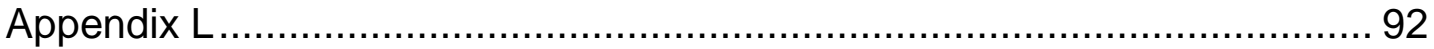

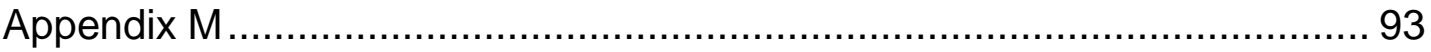

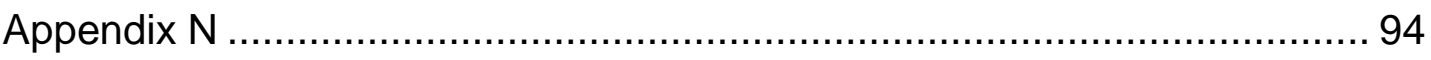

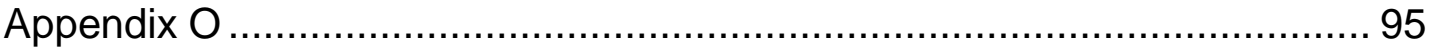




\section{List of Tables}

Table 1: Studies with Microfil casting compound ratios .................................. 29

Table 2: Tabular comparison of Microfil versus India ink ................................. 33

Table 3: Automation validation results ........................................................... 41 


\section{List of Figures}

Figure 1: Basic circulatory patterns of blood flow ..................................... $1 \mathrm{vi}$

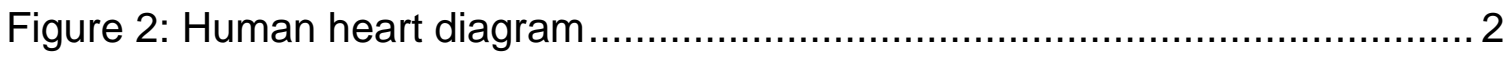

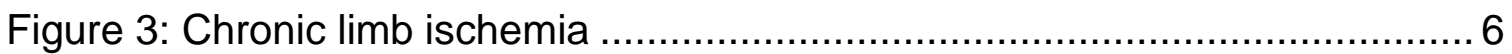

Figure 4: Diagram of optimal circulation through angiogenesis ..................... 12

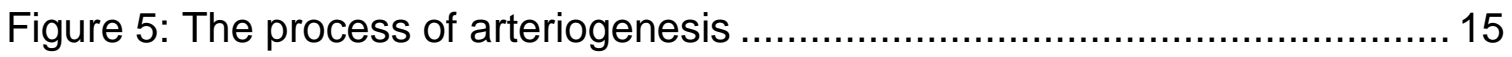

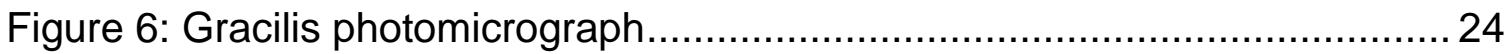

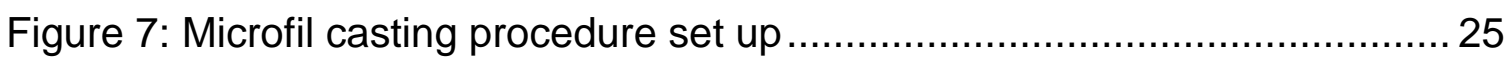

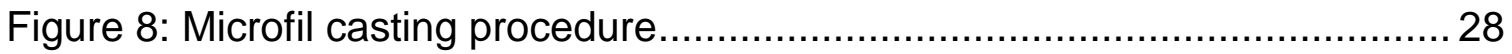

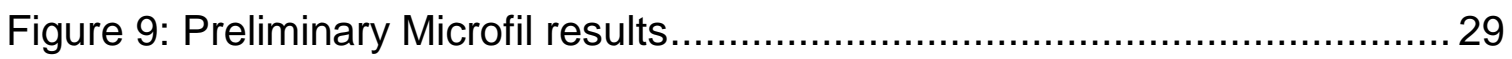

Figure 10: Initial Microfil viscosity adjustment results .............................. 30

Figure 11: Successful vascular cast using Microfil ................................ 30

Figure 12: Successful vascular cast using India ink .............................. 31

Figure 13: Microfil penetration at capillary level.................................... 32

Figure 14: How the automation program works ................................... 40

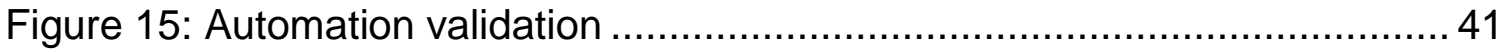

Figure 16: Macro versus manual measurement for length ......................... 43

Figure 17: Macro versus manual measurement for diameter ....................... 43

Figure 18: Automation macro on tortuous vessels ................................. 44

Figure 19: Bright field microscopy setup.......................................... 52

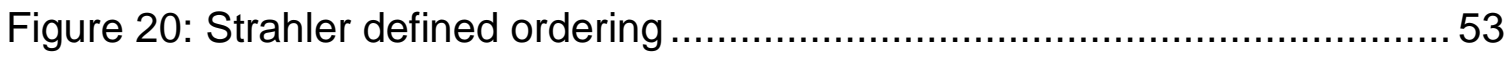

Figure 21: Successful vascular cast on ligated animal ............................ 55

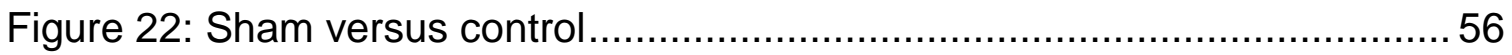




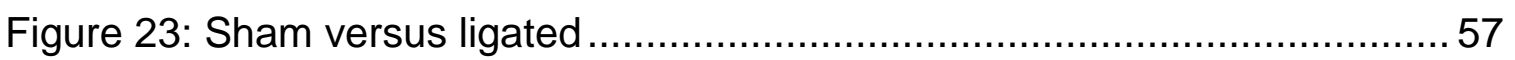




\section{Chapter 1 - Introduction}

\section{Normal Function of Circulatory System}

\section{Overview}

The primary function of the circulation is to carry out nutrient and waste exchange in all of the body tissues, and the rate of blood flow is controlled by tissue demand for oxygen and nutrients. Additional functions include the transport of endocrine factors, thermoregulation, and inflammation $(17,24,32)$.

The blood flow and circulation in the body are divided into the systemic circulation and the pulmonary circulation, the systemic circulation is also known as the peripheral circulation (Figure 1) (17).

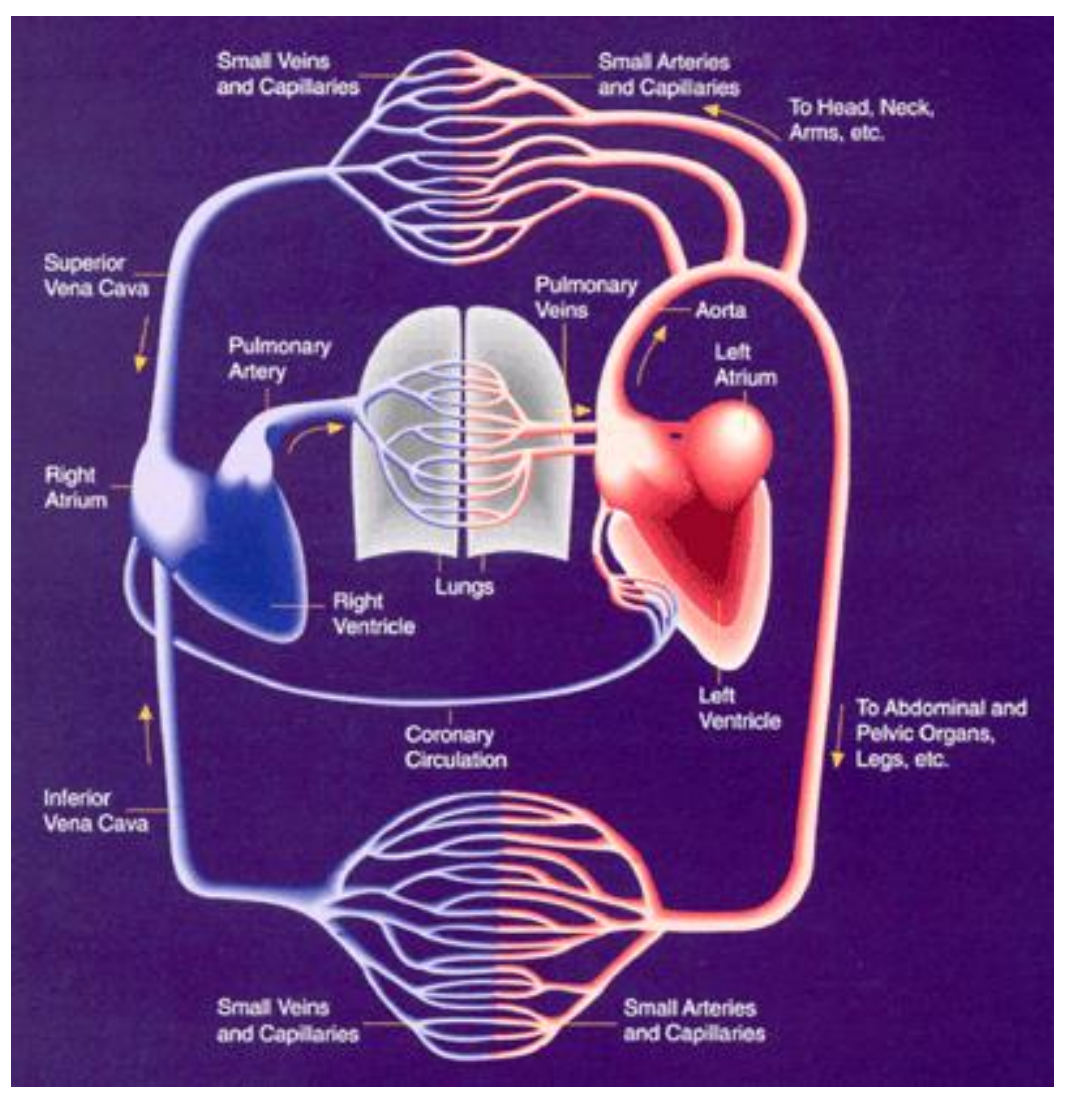

Figure 1 - Basic circulatory patterns of blood flow. Adapted from (9). 
The pump that creates the circulatory flow is the heart. The human heart is made up of 4 major chambers: the right atrium, right ventricle, left atrium and left ventricle $(17,32)$. The heart also has 4 valves; each valve is unidirectional and keeps blood in the heart flowing in the one direction. The valves are made up of two or three small but strong flaps of connective tissue called leaflets. The leaflets open to allow blood to flow through the valve and close to prevent blood from flowing backwards $(17,32)$, depending on blood pressure gradients across their location. The tricuspid valve is positioned between the right atrium and the right ventricle. The pulmonic valve separates the right ventricle from the pulmonary artery. The mitral valve is positioned between the left atrium and the left ventricle and the aortic valve separates the left ventricle from the aorta (Figure 2).

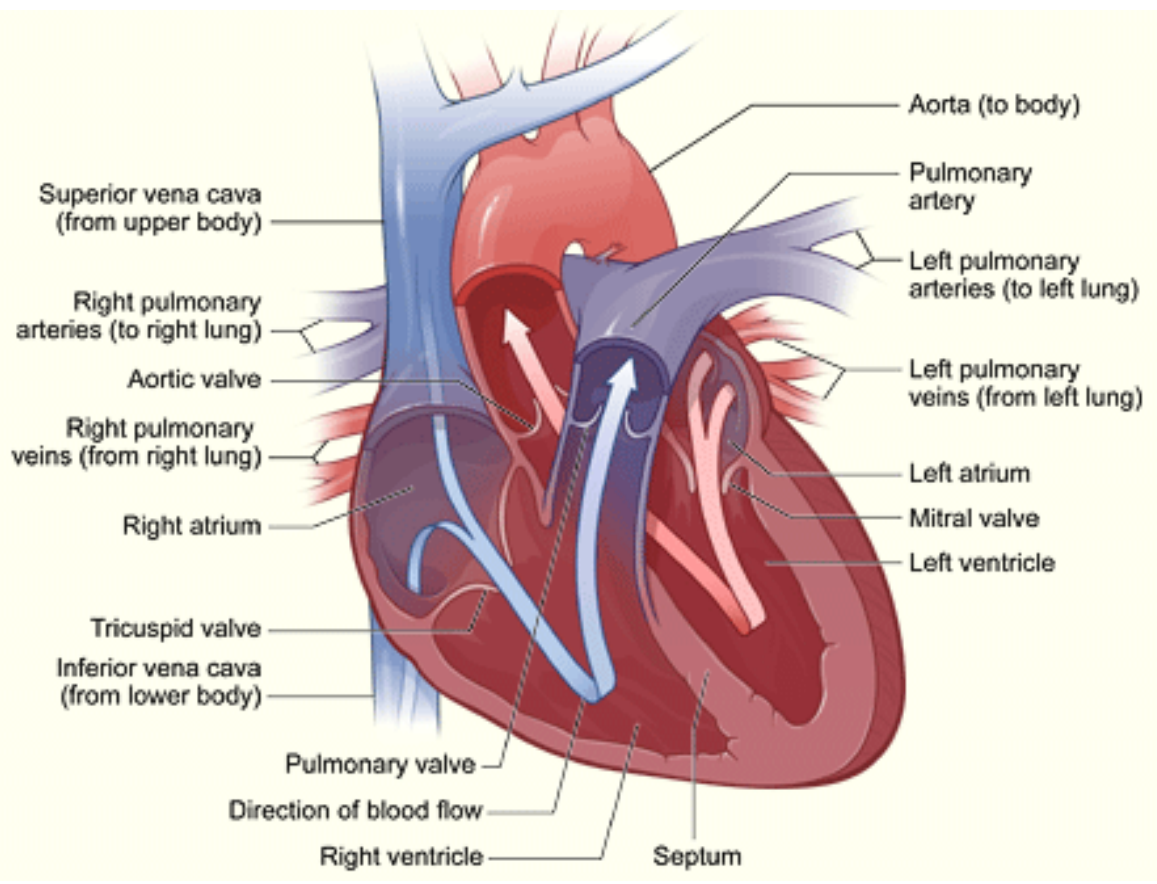

Figure 2: Human Heart Diagram, adapted from (21) 
The process of blood circulation begins with the right side of the heart receiving blood that is low in oxygen from the vena cava, which drains veins from all over the body. It then pumps the blood through the pulmonary arteries into the lungs where it will become oxygenated. The oxygenated blood then leaves the heart from the aorta and travels through a branching system of arteries and arterioles; from the arterioles the blood travels into capillaries, the site of nutrient and waste exchange, before moving into venules; the venules gradually coalesce into veins, which bring the deoxygenated blood back to the vena cava and right atrium to form a continuous circulation $(3,12,17)$. At each order of branching, the sizes of the vessels decrease but the number of individual vascular entities increases $(12,17)$ and all blood in the human body will traverse the full circuit an average of once each minute when the body is at rest and as many as six times each minute during physically activity (17).

The role of the arteries is to transport blood under high pressure and velocity to the tissues, compared to the veins, which transport blood at high velocities but low pressures and thus form the capacitance vessels of the circulation (as much as $60 \%$ of a person's blood volume can be found in the veins at rest). The arterioles are the last of the small branches of the arterial system before blood flows into the capillaries (32), the primary site of nutrient and waste exchange between the blood and the interstitium/parenchyma. The venules then collect the blood from the capillaries as they gradually coalesce into veins. Veins take the deoxygenated blood back to the heart to be oxygenated by the lungs and also serve as a major reservoir of extra blood (17). 


\section{Microcirculation}

As stated earlier, the most important functions of the circulation occur in the microcirculation. This is due to the fact that the microcirculation is the site of nutrient and waste exchange. Capillary blood flow is determined at the upstream arterioles, based on the contractile state of smooth muscle cells that encircle the arterioles. Smooth muscle cells control arteriolar diameter by either contracting to constrict arterioles and decrease blood flow, or relaxing to dilate the arterioles and increase blood flow. Arterioles have the potential to drastically changing the blood flow in response to tissue needs (17) because according to Poiseuille's Law, diameter is the largest contributing factor to blood flow: $F=\frac{\pi \Delta P r^{4}}{8 \eta l}$, where $\mathrm{F}$ is the rate of blood flow, $\Delta \mathrm{P}$ is the pressure difference between the ends of the vessel, $r$ is the radius of the vessel, $\eta$ is the viscosity of the blood, and I is the length of the vessel. It is important to note that blood flow is proportional to the fourth power of the radius of the vessel, which is why it is the largest factor when determining blood flow for the tissue for which it is supplying blood (17). The arterioles will branch until they reach diameters of 5 to 9 micrometers where they transition to capillaries (32). The walls of the capillaries are extremely thin, constructed of single-layer, unicellular endothelial cells, which are surrounded by a very thin basement membrane on the outside of the capillary (32). Although it isn't necessarily continuous like arteriolar smooth muscle, capillaries also have perivascular cells that surround the endothelium, but do not contain smooth muscle contractile proteins like arterioles $(17,32)$. The total thickness of the capillary wall is only about $0.5 \mu \mathrm{m}$. The internal diameter of a capillary is 4 to 9 
micrometers, barely large enough for red blood cells and white blood cells to squeeze through $(17,32)$. Therefore, water, cell nutrients, and cell waste can all interchange quickly and easily between the tissues and the circulating blood. Diffusion is the most common way that substances are transferred between the interstitial fluid. If a substance is lipid soluble, like oxygen and carbon dioxide, in specialized microcirculations, such as the liver and kidney, it can diffuse directly through the cell membranes of the capillary without having to go through the pores. This process is much faster than for substances like sodium ions and glucose that are lipid-insoluble and must diffuse through the inter-cellular junctions or endothelial transport proteins $(10,17,35)$. The human body has about 10 billion capillaries with a total surface area estimated to be around 500 to $700 \mathrm{~m}^{2}$ with a total cross-sectional area of $2500 \mathrm{~cm}^{2}$, while the total cross-

sectional area of the aorta is only $2.5 \mathrm{~cm}^{2}(3,17,24)$. This is why branching plays such a crucial role in efficient blood delivery; it allows the body to effectively deliver crucial nutrients and expel harmful waste over a large area from a small initial cross-sectional area.

\section{Dysfunction in the Circulatory System}

\section{Peripheral Artery Disease}

Ischemic vascular diseases are the most common cause of death in the western world (19). The vascular endothelium is sensitive to changes in physical forces (e.g. shear) or signaling molecules, both of which can result in pathology (24). 
Peripheral arterial disease (PAD) is an obstructive arterial disease that causes ischemia in the lower extremities during exercise or, in more severe cases, during rest $(46,50)$. PAD is a highly prevalent atherosclerotic disease, most common in aged populations $(20,46,50)$, affecting 8 to 12 million Americans. PAD is associated with significant morbidity and mortality $(20,50)$, mostly due to a significant increase in coronary artery disease in these patients. PAD can be categorized as either intermittent claudication, which involves pain during physical activity, or critical limb ischemia, which involves a severe blockage in the arteries of the lower extremities that results in pain at rest $(20,46$, 50). Complications can include chronic ulcerations in the legs and feet and if left untreated, the complications of critical limb ischemia will result in amputation of the affected limb (Figure 3).

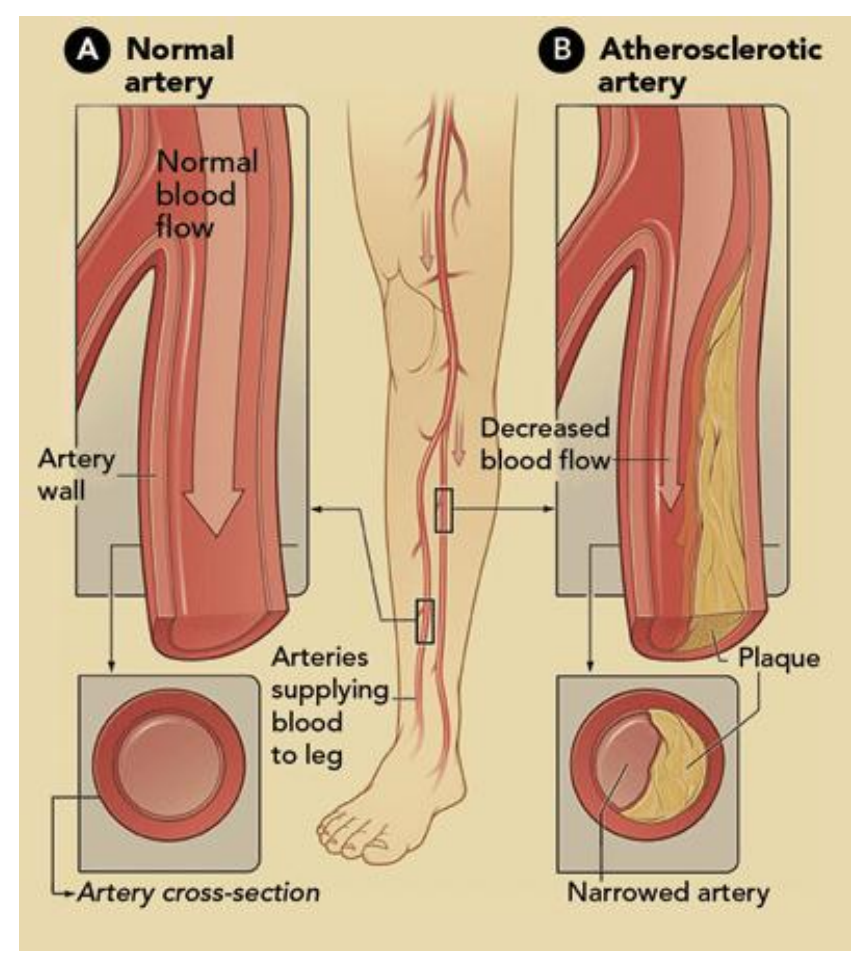

Figure 3 - Chronic limb ischemia. Adapted from (22). 
The pathogenesis of PAD is due to atherosclerosis $(17,20,32,37,46,50)$. Atherosclerosis is a disease of the medium and larger sized arteries in which fatty lesions called atheromatous plaques develop on the endothelial surfaces of the arterial wall $(17,32)$. Endothelial dysfunction is a precursor to atherosclerosis and can be measured before plaques form. Endothelial dysfunction involves the increased expression of adhesion molecules and a decrease in nitric oxide and prostacyclin that normally prevent adhesion of platelets and monocytes $(2,17$, 41). Circulating monocytes and lipids then aggregate at the site of the intimal injury; eventually the monocytes extravasate and differentiate into macrophages that phagocytose oxidized lipoproteins that have accumulated in the subintimal space $(2,41)$. Ingestion of the lipoproteins by macrophages will lead to a fatty streak and with time the fatty streak will grow larger and larger, which will cause the surrounding smooth muscle and fibrous tissues to proliferate to form plaques $(2,17)$. In addition, macrophages also release inflammatory cytokines that stimulate smooth muscle cell proliferation and expansion of fibrous tissue in the subintimal space $(2,41)$. The cellular proliferation coupled with the lipid deposits can become so large that the plaque bulges into the lumen of the artery and reduces blood flow; severe cases will completely occlude the vessel (17). Normally, arteries are very elastic, but eventually atherosclerosis causes arteries to become stiff, referred to as "hardening of the arteries," due to connective tissue accumulation and calcium salts precipitating with cholesterol and other lipids causing hard calcifications (17). Once the arteries have hardened, the plaques they contain are at risk for rupture; their rough surface also protrudes 
into the flowing blood, causing blood clots to develop that block the flow in the artery $(17,32,41)$. Patients with PAD have a much higher risk of coronary heart disease; therefore the treatments aim to decrease cardiovascular risk while also restoring the patient's quality of life by reestablishing blood flow to the ischemic $\operatorname{limb}(20,46)$.

\section{Patient Treatment}

Most of the treatments for PAD are designed as secondary prevention for cardiovascular risk. These treatments include: supervised exercise, smoking cessation treatments for patients that are smokers, antiplatelet therapies, blood glucose control for patients with diabetes, and lipid-lowering agents $(20,37,46)$. Other more serious treatments that address primary risk include percutaneous coronary re-vascularizing interventions, coronary bypass surgeries, endarterectomies, peripheral stenting and by-passing. In 1999, in the US alone, there were 1.1 million percutaneous coronary revascularizations, 355,000 coronary bypass surgeries and 131,000 endarterectomies (19). These serious treatments have their limitations and risks, for example, not all patients with coronary and peripheral vascular disease are able to receive these therapies due to age or unavailability of a vessel for a bypass. Also, about one third of patients will develop restenosis after treatment, and the neurological risk for patients that need to be placed on cardiopulmonary bypass for their surgery is high (19). While all of these treatments are somewhat successful they fail to stimulate the 
vasculature's natural repair process of arteriogenesis and angiogenesis to restore blood flow to the ischemic limb.

Therapeutic angiogenesis and therapeutic arteriogenesis were proposed as treatment strategies in the early 1990 s as an alternative to surgical or endovascular revascularization therapies. The goal of therapeutic angiogenesis/arteriogenesis is to treat ischemic disease by stimulating the natural revascularization processes of the body. This would cause a restoration of blood flow to an occluded tissue, which is an important but challenging goal as there are several hemodynamic, metabolic as well as cellular and molecular signaling factors involved in the mechanisms of structural remodeling and adaptation in the microcirculation $(24,29,30)$. The ultimate goal is to understand the mechanisms of vascular growth in order to develop treatments to stimulate arteriogenesis and angiogenesis as a single therapy or even as an addition to other surgical procedures to achieve a more complete level of revascularization for both macrovascular and microvascular diseases.

\section{Vascular Growth}

There are many different aspects to vascular growth, which can be separated into three categories: vasculogenesis, angiogenesis and arteriogenesis. Each of these aspects can then be broken down further into different entities with different mechanisms, which will be discussed later. Understanding each of these aspects is crucial for developing tests and testing strategies that help create efficacious treatments. 


\section{Angiogenesis}

Angiogenesis is defined as the growth and development of new capillaries from existing capillaries and is a normal process critical to development, wound healing, reproduction, and exercise adaptation $(3,12,15,19,24,38,51)$. It is also involved in inflammatory diseases such as rheumatoid arthritis, psoriasis, and tumor growth (19). Angiogenesis is categorized by two different types of vessel growth: sprouting angiogenesis and intussusceptive angiogenesis $(12,40)$. In the embryo, the vascular system begins with vasculogenesis, the de novo creation of capillaries by endothelial and perivascular cells. Vasculogenesis (Figure 4A) is followed by a sprouting phase (Figure 4B) characterized by the appearance of multiple capillary sprouts that then fuse to form the primary capillary plexus (Figure 4C) that is remodeled into an optimal circulation by intussusception (12, 13, 24, 40). In the adult, sprouting angiogenesis occurs primarily in response to hypoxia while intussusception is stimulated by increased shear stress $(12,13,18,40)$.

Intussusceptive angiogenesis involves the formation of new capillaries by the development of tissue pillars in the capillary lumen and expansion of those lumens to form new capillary networks $(12,38,51)$. Further, both vascular growth and remodeling occur mostly by intussusception $(12,13)$. The distinguishing factor that separates sprouting angiogenesis and intussusceptive angiogenesis is the formation of a tissue pillar into the vascular lumen. There are four consecutive steps in pillar formation: first, a zone contact is established between opposite capillary walls; second, there is a reorganization of the inter-endothelial 
cell junctions and central perforation of the bilayer; the third step is the formation of the interstitial pillar core; and the final step is the increase in girth of the pillar $(3,12)$. Intussusceptive angiogenesis is pervasive and can occur in many organs during normal (cerebral vascularization after stroke) and pathological (tumor angiogenesis) microvascular growth in order to establish normal branching patterns $(12,38)$.

Once the pillar formation has occurred there are three distinct elements that define intussusceptive angiogenesis. The first is intussusceptive microvascular growth, which can be described as the expansion of the capillary plexus (12). The next element is intussusceptive arborization, which is the formation of a vascular feeding tree. The original capillary plexus formed by vasculogenesis and sprouting doesn't resemble a tree-like branching pattern, where larger arteries branch into progressively smaller and smaller diameter conduits, which is the most efficient flow delivery scheme. Intussusceptive arborization is the process by which the capillary plexus is transformed into a tree-like branching pattern after intussusceptive microvascular growth has taken place. Intussusceptive arborization is initiated by the formation of compacted vertical pillars; these pillars undergo reshaping into narrow tissue septa that progressively fuse to delineate a new vascular entity (12). The final element is intussusceptive branching remodeling, which is how the vascular tree geometry is optimized (13). The function of intussusceptive branching remodeling is to optimize hierarchy and flow properties within the system by minimizing power consumption and maintaining a constant shear stress, this occurs by changes in 
branch angle through the formation of transluminal pillars near bifurcation sites of arteries and veins (13). Another aspect of intussusceptive branch remodeling is the removal of branches that are unnecessary by a mechanism called vascular pruning, or regression (38), where large numbers of smaller vessels are reduced for few larger sized ones (Figure 4D) $(4,12,13)$. The cause of pruning can be attributed to hyperoxia, which causes VEGF to be down-regulated and unnecessary vessels to be pruned (38).

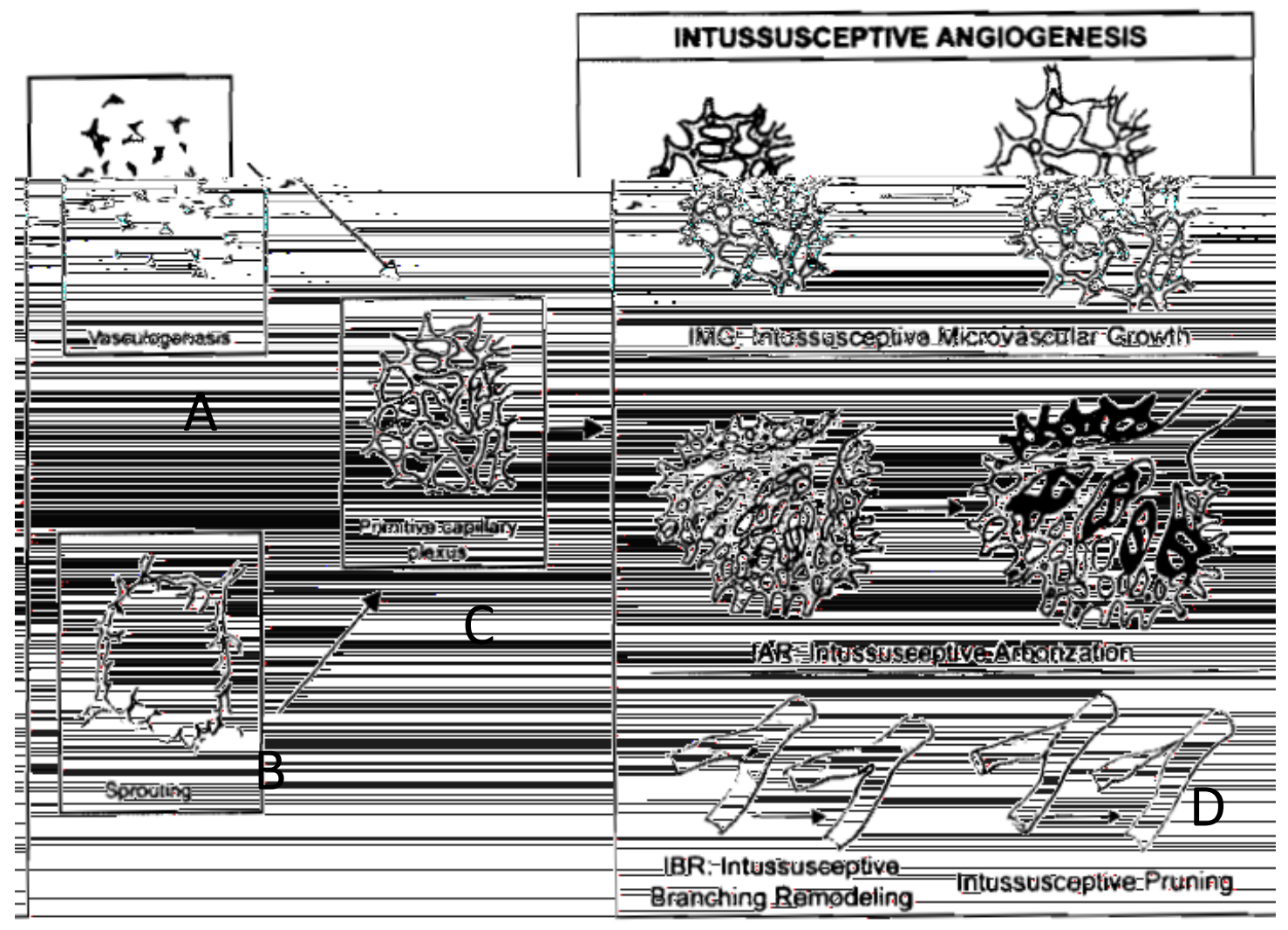

Figure 4 - Diagram of optimal circulation through angiogenesis. A)

Vasculogenesis is the creation of capillaries by endothelial and perivascular cells. B) Sprouting marks the appearance of multiple capillary sprouts that then fuse to form the C) primary capillary plexus. Intussusceptive angiogenesis then begins with intussusceptive microvascular growth (IMG), or the rapid expansion of the capillary plexus. Next, intussusceptive arborization (IAR) forms the vascular feeding tree. Finally, intussusceptive branching remodeling (IBR) optimizes branching geometry through branch angle adjustments and D) pruning. 
The effectiveness of pillar formation is dependent on blood flow rate, and the faster the blood flow rate is, the faster the pillar will form, and it is a process that occurs with minimal amounts of endothelial cell proliferation, while also maintaining low vascular permeability. In contrast, sprouting angiogenesis is a drawn out process that requires extensive proliferation of endothelial cells and an increased vascular permeability, which expends more energy (12).

\section{Arteriogenesis}

Angiogenesis is the formation of new capillaries by sprouting and intussusception from pre-existing architecture, and vasculogenesis is the embryonic development of blood vessels from angioblasts (4). Arteriogenesis, on the other hand, is a different process by which a small arteriole chronically increases its diameter through outward remodeling $(4,38)$. Angiogenesis and arteriogenesis have different initial pathways as well as different results; the main difference is that arteriogenesis is more effective than angiogenesis in replacing an occluded artery by returning normal blood flow to an ischemic tissue (4). The outward remodeling occurs through endothelial cell activation, vascular cell proliferation, and rearrangement of the extracellular matrix (38).

Increased sheer stress is a trigger for increases in arterial diameter (flow induced dilation), and with the change in flow that persists, outward remodeling of the arterial wall will occur to normalize hemodynamic shear stress $(4,19,38)$. It makes sense then, that the maximal arteriogenesis occurs in situations at maximal sheer stress (4). The increased flow increases the arterial wall tension 
and activates the endothelium (4). What is still unclear is how the increase of sheer stress is being "sensed" by the cells of the vascular wall $(4,19)$. The endothelium play a crucial role in the remodeling response to sheer stress as the first sign of arteriogenesis after arterial occlusion is that the endothelial cells of collateral vessels appear swollen and are randomly oriented and do not line up in the direction of blood flow (4). Endothelial cells are able to react to different sheer stress gradients by activating multiple transcription factors (19). Once the endothelium is activated, there is an increase in endothelial nitric oxide (NO) synthase, monocyte chemoattractant protein (MCP-1), transforming growth factor beta (TGF- $\beta$ ), as well as intercellular adhesion molecule-1 (ICAM-1) and vascular cell adhesion molecule (VCAM) $(4,19,43)$. The up-regulation of MCP-1 seems to be important for the attraction of monocytes to the endothelium and their aggregation around growing collateral vessels $(4,19)$. The effects of NO are not yet understood, but it has been found to dilate collateral vessels, which decreases shear stress. NO can also activate metalloproteinases (MMPs) and may play a role in cell migration and proliferation, on the other hand, NO can also have inhibitory effects on endothelial, vascular smooth muscle cells (VSMC), atherogenesis, and inflammation (19). There is also an increased permeability of the endothelium, as can be seen from the adherence of monocytes, and leakage of platelets, plasma proteins and erythrocytes $(4,42)$. Other molecules that have been found to be involved are MMP-2, FAK, and bone marrow tyrosine kinase (4), which are involved in cell proliferation and migration (4). 
Increased shear stress also changes VSMC during arteriogenesis by converting smooth muscle cells (SMC) between contractile (physiological phenotype) and synthetic (proliferative phenotype) $(4,47)$. The cellular signals to mobilize the SMC come from the degradation of the basement membrane, and the internal and external elastic laminas, which act as barriers to prevent outward remodeling of the vessel. In order for this degradation to occur MMP-2 and MMP9 are up-regulated in the SMC, which causes the basement membrane and the internal and external elastic laminas to become fragmented to allow for collateral vessel growth (4). Other signals come from transcriptional regulators like ERK and FAK, which promote cellular migration (4). The mechanical strain from the occlusion, increased sheer stress and growth factor stimulation lead to the autophosphorylation of FAK and ERK to promote the migration and proliferation of VSMC so that arterioles' successfully transform into collateral vessels (Figure 5) (4).

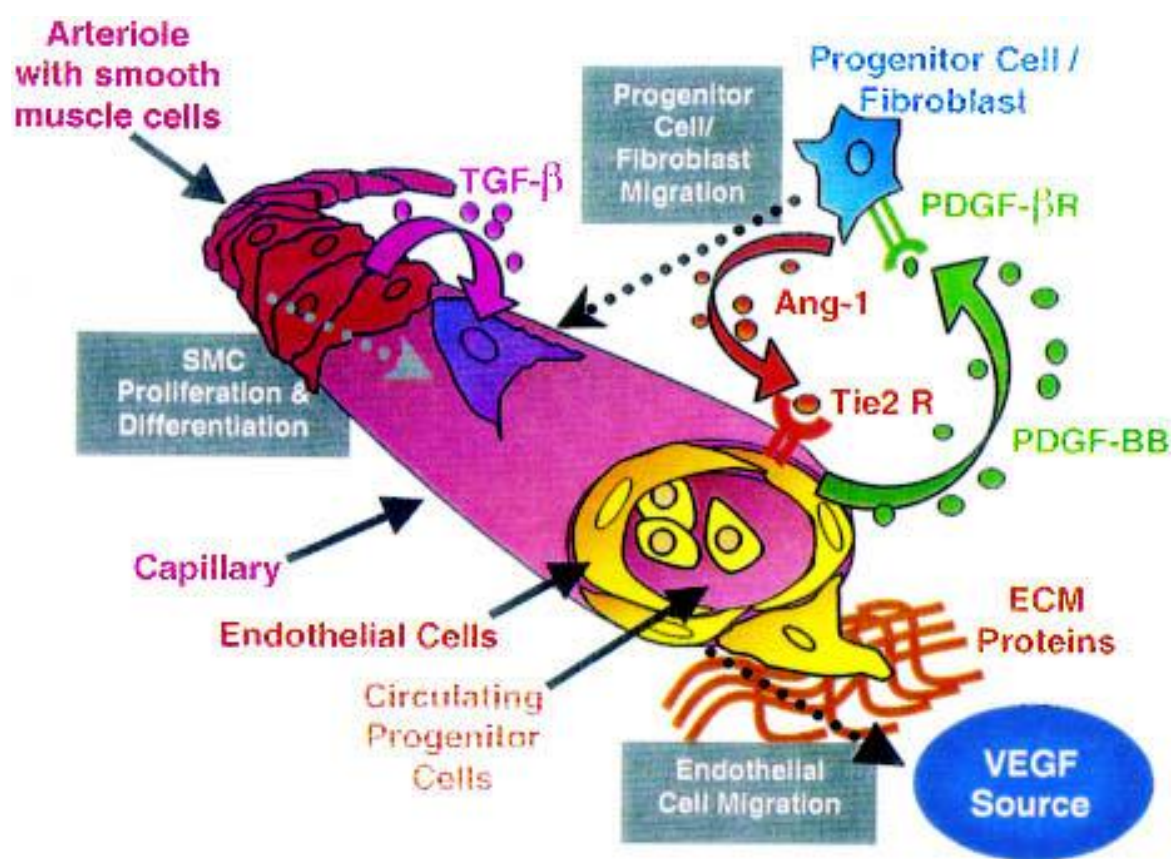

Figure 5 - The process of arteriogenesis. Adapted from (38). 
It is important to appreciate that arteriogenesis is more effective than angiogenesis in replacing an occluded artery by returning normal blood flow to an ischemic tissue (4) and understanding the entire process is crucial to advancing the field of therapeutic arteriogenesis for diseases like PAD.

\section{Conclusion of Vascular Growth}

All of the remodeling processes are a result of complex and coordinated cellular interactions that are brought about by cell-to-cell and cell-to-matrix communication from environmental stimuli, so that constant blood vessel remodeling occurs (38), where network structure is remodeled to match tissue requirements . Understanding how vascular growth works and how different therapies affect the long-term stability and survival of tissue in patients with ischemic diseases is critical to designing both revascularization therapies for ischemic disease (and anti-angiogenic therapies for cancer) $(38,51)$. As we progress in therapeutic arteriogenesis and angiogenesis there are important considerations that need to be made: delivery methods and agents need to be effective in stimulating arteriogenesis but also have acceptable levels of risk, e.g. plaque instability, plaque growth, or an inflammatory response. We must weigh success from our vascular growth treatments with treatment failure from tumor growth or other inflammatory diseases, and decide how much of that risk is acceptable when searching for success (19). As we move forward and continue to gain insight into vascular growth and the role that it plays in other diseases, there is the potential that this type or research could change the face of medicine 
in the next decades, with more than 500 million people worldwide predicted to benefit from pro or anti-angiogenesis treatments (15).

\section{Attempts to Stimulate and Assess Vascular Growth}

It is important in the development of treatments that we are able to both stimulate and assess vascular growth. With this goal come many challenges as we make progress toward developing vessel maturation therapies for therapeutic angiogenesis. One hope for patients who are suffering with various ischemic diseases is the ability to form vascular networks in vivo by delivering angiogenic or arteriogenic molecules as recombinant proteins using controlled-release devices or as genes using viral vectors or plasmid DNA (23). Results from clinical trials using these techniques have shown that the formation of a mature vascular network will require very precise regulation of a large number of both angiogenic stimulators and inhibitors (24). For example, clinical trials for limb ischemia using VEGF and FGF yielded less than optimal results, as some patients had successful limb salvage while a majority of the patients treated with VEGF developed transient limb edema, which is consistent with VEGF enhancement of vascular permeability (1). Preclinical experiments using a controlled release device or an adenoviral vector to deliver basic FGF or VEGF led to the transient development of stable vascular networks, which after time began to resemble an immature tumor vasculature $(11,36)$. Therefore, more precise understanding of the vascular growth process through the use of animal models is necessary before therapeutic angiogenesis will be successful. 
Many follow up experiments have focused on a more precise approach as the lack of positive trial data has focused researchers and clinicians on the fact that arteriogenesis and angiogenesis are complex processes and simply supplying vascular growth factors to ischemic tissue does not provide effective therapy (49).

One approach to improve the effectiveness of therapeutic angiogenesis is to deliver a cocktail of angiogenic molecules to the desired site in a spatiotemporal manner that more accurately resembles their endogenous production (24). Polymeric delivery systems can mimic the endogenous release of two or more growth factors. For example, controlling the dose and rate of delivery for a VEGF and platelet-derived growth factor (PDGF) combination therapy leads to vessels that appear more mature than those formed using VEGF alone (39). These results moved researchers to find out the minimal number and type of molecules needed to produce stable vascular expansion and to figure out how to optimize delivery in order to effectively treat ischemic disease. Another approach is to deliver a transcription or growth factor that regulates expression of the various growth factors required for mature vessel formation. Hypoxia-inducible factor (HIF)-1 $\alpha$ is responsible for regulating the expression of a large number of pro- and antiangiogenic genes and it enhanced perfusion in an ischemic rabbit hind-limb model $(45,48)$.

To be truly effective, once mature vessels have been established, therapeutic angiogenesis must successfully create a mature network structure. A mature network involves network remodeling and vascular reactivity. Network 
remodeling involves arborization and pruning to create an efficient network (12, 31), while vascular reactivity is important for regulating blood flow in response to tissue needs (26). The ultimate goal of therapeutic angiogenesis is to revascularize ischemic tissue, which requires a mature vasculature and a mature network that will restore tissue oxygenation levels in response to the chronically insufficient blood supply.

The therapies described above are moving towards creating more mature vessels but little work is being done to create more mature networks, which is due to the difficulty of assessing a network. Most often, network maturation is assessed by counting vessels in a histological cross section, which gives little information on what the overall network looks like. In order to create more effective therapies it's important to understand how to create more mature networks.

Several morphological parameters such as the diameters of blood vessels, blood path length, vessel length, branching characteristics, and vessel quantity within a vascular network describe the architecture of the microcirculation. Following ischemia, these morphological parameters change. Diameters increase in response to the elevated shear stress, blood path length increases as blood is re-routed, which consequently causes a change in the branching characteristics of the network because of the structural remodeling and changes in flow direction. All of these changes could be important when developing therapies; however, researchers in this area typically ignore network topology. Instead of focusing on one morphological parameter, such as vessel number, it 
would be beneficial to examine as many as possible in order to evaluate the vascular network as a whole. The microcirculation is critical to nutrient and waste exchange and changes could affect muscle functionality; the overall goal of this research was the changes in morphological parameters following arterial occlusion.

\section{Objectives/Hypothesis}

Currently, our lab uses a manual process to gather morphological data for our experiments. The morphological data is used in a model to predict hemodynamics and calculate vascular morphometry in normal and remodeled microcirculatory networks. This study aims to establish a system that will expedite the process of gathering morphological data needed for the model and to examine the impact of arterial occlusion on morphology. More specifically, this study had three distinct objectives to reach the goal:

(1) Create a vascular casting protocol to only cast the arterial side of the microcirculation. Previous work filled both the arterial and venous sides, even though only the arterial side was measured. The venous fill increased the difficulty of measurement and tracing and provided no additional value to the research.

(2) Decrease the amount of time it takes to measure the microvasculature of the anterior gracilis muscle through some form of automation. Previous work used completely manual measurements to gather data and I sought to expedite this data-gathering process. 
(3) Determine the effects of elevated flow in a collateral artery on branching arteriolar network topology. This aim utilizes the proposed methods to demonstrate proof-of-concept and to determine if collateral remodeling was associated with remodeling in the arteriolar side branch trees. 


\section{Chapter 2 - Development of an Arterial-Specific Vascular Casting Procedure}

\section{Introduction}

Efficient morphological assessment requires a multi-step tissue preparation process in order to collect data for analysis and for input into our model. The process of sample preparation can be summarized in 5 steps: vascular casting, dissection, dehydration, clearance, and sample mounting. Improving the vascular casting protocol could optimize the entire process of gathering data for the model. The goal of vascular casting is to fill the circulation with a high contrast agent, which generally requires clearing the circulation of blood. The original protocol for vascular casting utilized nontoxic India ink(16). Although this protocol is successful in achieving high contrast between the vasculature and surrounding tissue, there are some drawbacks to casting with India ink and the subsequent morphological analysis, which include: India ink remains in a liquid state, so leakage occurs if the vessels aren't cauterized properly during muscle dissection; India ink takes a significant amount of time to dialyze, filter, and purify in preparation for injection; and India ink casts both the arterial and venous side of the circulation. I aimed to resolve these three issues to improve data collection. The solutions formulated for visualizing only the arterial side were to increase the viscosity of the casting agent to prevent it from traversing the capillary level by adding dextran to the casting compound or to use a fluorescent marker to label the arterial side of the circulation, so that when imaged the arterial side is expressed with fluorescence. While researching 
different casting agents, I found a group that was able to successfully cast the spinotrapezius vasculature of a rat using Microfil (27). Further research into Microfil demonstrated that it was a radiopaque, polymerizing silicone rubber compound with variable viscosities that could be used to cast the arterial side of both a rat and mouse kidney (33). This previous work was successful using Microfil to cast the arterial side and led me to begin protocol modification with Microfil instead of modifying the India ink or using fluorescence.

\section{Materials and Methods}

Microfil is a casting agent with variable viscosity, composed of a colored compound, diluent, and curing agent. The optimal Microfil viscosity for arterial casting of the gracilis muscle was investigated using a murine model. All protocols were approved by the Institutional Animal Care and use Committee. Male Swiss Webster mice weighing 25-35g from the California Polytechnic State University Vivarium were used to optimize the Microfil casting protocol, 8 male C57BL/6 mice were used to validate the consistency of the protocol, and another 8 male C57BL/6 mice were used to assess the impact of arterial ligation on microvascular morphology (presented in Chapter 4).

The longitudinal gracilis arcade is the main blood supply for the gracilis muscle, and is fed by the profunda femoris of the femoral artery (medial side) and the saphenous artery (lateral side) (16). The gracilis arcade runs parallel to the muscle fibers over almost the entire length of the muscle (16) (Figure 6). 


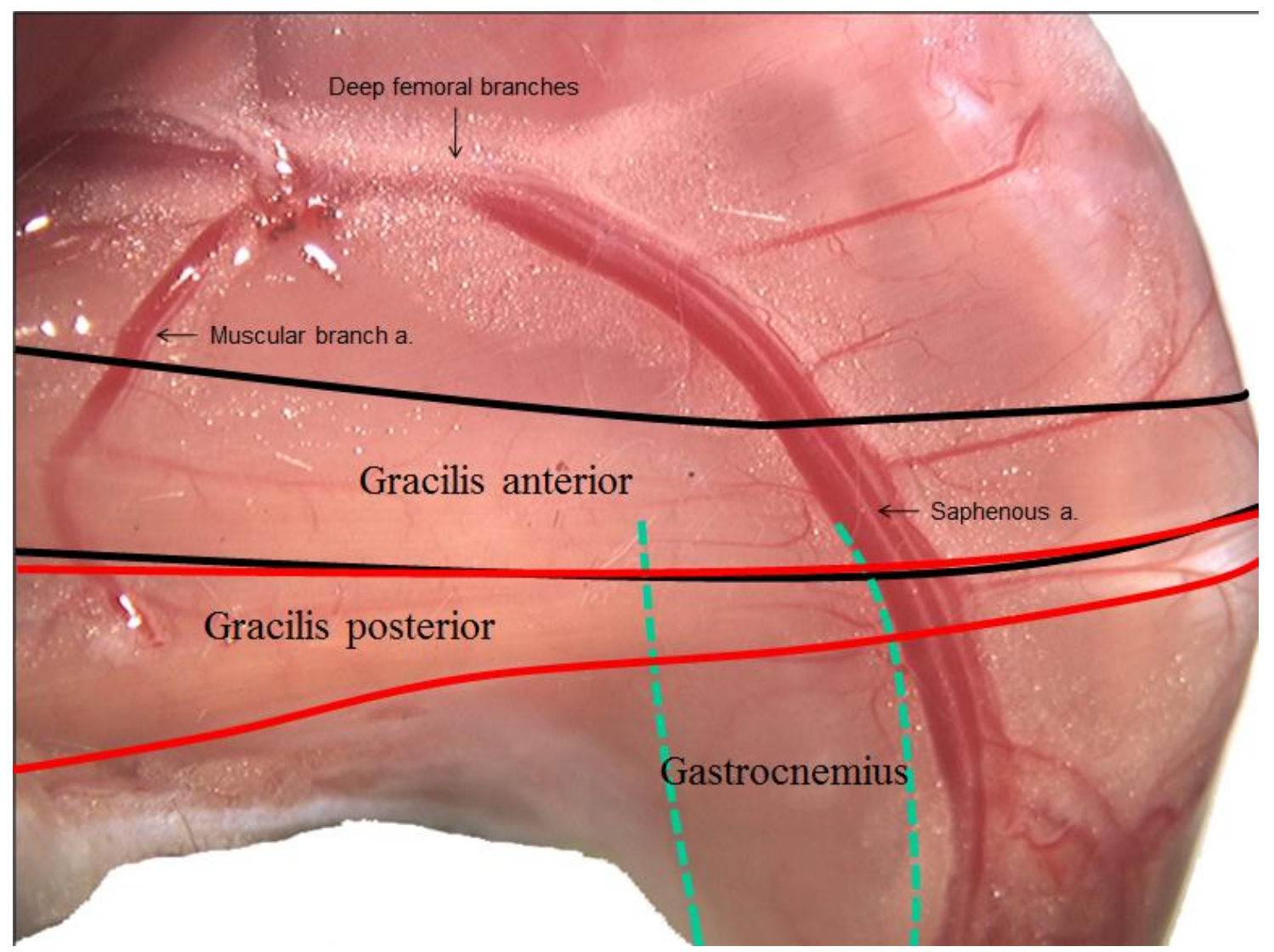

Figure 6 - The gracilis artery runs through the gracilis anterior and is fed by the profunda femoris and the saphenous artery.

Completing a vascular cast with Microfil requires the preparation of surgical instruments and materials. These included forceps, bone-cutting scissors, iris scissors, hemostats, microdissection scissors, a vascular clamp, surgical tape, $20 \mathrm{ml}$ syringe, bench cover, heating pad, catheter/stopcock/blunt needle apparatus containing a PE-100 catheter, isoflurane anesthetic, and gauze sponges (Figure 7). A vasodilator cocktail is also necessary to flush out the blood and maximally dilate the vasculature before injecting the casting agent. 


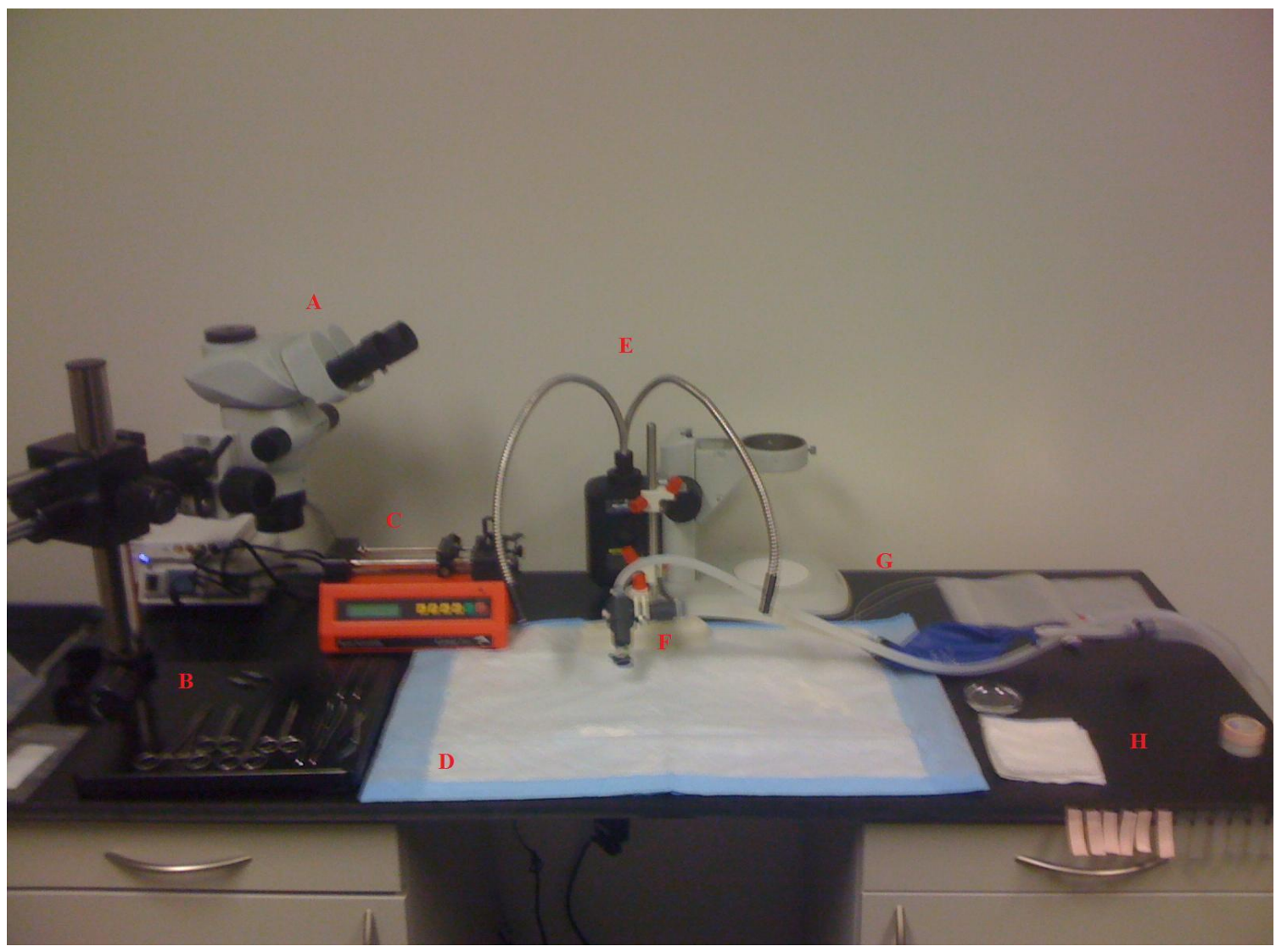

Figure 7 - Microfil casting procedure set up. A) Light microscope, which can be rotated in to check on the progress of the vascular cast. B) Surgical instruments Hemostats (1), Bone scissors (1), metzenbaum scissors (1), Iris scissors (1), forceps (2), microdissection scissors (1), vascular clamp (1). C) Syringe pump. D) Bench Cover with heating pad underneath. E) Octopus light source. F) Isoflurane nose cone. G) PE-100 catheter. H) Miscellaneous items-Saline, gauze, tape.

The cocktail consists of heparin, adenosine and sodium nitroprusside, which are all combined with PBS solution and heated to $37^{\circ} \mathrm{C}$ in a water bath. The Microfil mixture is created by vortexing $2 \mathrm{~mL} \mathrm{MV}-122,1.25 \mathrm{~mL} \mathrm{HV}$-Diluent and $1.25 \mathrm{~mL}$ MV-Diluent into a $14 \mathrm{~mL}$ conical and placing in the $37^{\circ} \mathrm{C}$ water bath (Figure $8 \mathrm{~A}$ ). Once the prep work was done the animal was weighed and anesthetized with isoflurane and laid supine upon a heating pad to maintain core body temperature. Hindlimb hair was removed with clippers and depilatory cream (Figure 8B). The 
animal was taped down to the heated bench cover and the skin was separated from the superficial abdominal and thoracic musculature. Once the skin was removed, the $20 \mathrm{~mL}$ syringe was filled with warm vasodilator cocktail, loaded into the syringe pump, and attached to the catheter. An incision was made through the abdomen, close to the diaphragm before the ribs and diaphragm were quickly transected and reflected with hemostats to expose the chest cavity (Figure 8E). Due to pneumothorax, the ventricular catheterization must be done quickly before blood begins clotting, which would ruin the clearance and vascular cast. Excess tissue was cut away from around the heart and a small incision was made in the apex of the left ventricle. The catheter was inserted through the incision into the left ventricle and clamped in place with a vascular clamp (Figure 8C). Once the catheter was in place the right atrium was cut and the syringe pump was started in order to inject the vasodilator cocktail at $5 \mathrm{ml} \cdot \mathrm{min}^{-1}$. The skin above the gracilis muscle was removed to visualize vascular clearance before refilling the $20 \mathrm{~mL}$ syringe to continue flushing the vasculature (Figure $8 \mathbf{G}$ ). Once the entire vasodilator cocktail had been injected, $.2 \mathrm{~mL}$ of curing agent was added to the Microfil mixture to start the polymerizing reaction (it is crucial to wait until the last possible moment to add the curing agent when preparing the Microfil mixture to maintain the compound in a liquid state during perfusion). The Microfil mixture was inserted into the syringe pump and perfused at $.5 \mathrm{~mL}$ per minute (Figure 8D). The hindlimb was monitored under the microscope until the Microfil had passed the knee. At this point the syringe pump was stopped (approximately $1 \mathrm{~mL}$ of Microfil had been perfused) and a tourniquet was tied around both ankles 
with silk suture to prevent entry of the Microfil in the venous circulation through A$V$ shunts near the ankle (Figure 8F). The Microfil perfusion was resumed, and continued until the casting agent spilled out of the right atrium or until the cast was deemed satisfactory by visual inspecting the gracilis under the microscope. The catheter was removed, but the heart clamp was left to prevent any Microfil leakage, and the mouse was allowed to sit undisturbed for at least 10 minutes while the Microfil begin curing (Figure $8 \mathbf{H}$ ). The open wounds were covered with plastic wrap and the mouse was placed in a plastic bag to prevent desiccation while the animal was left overnight at room temperature to allow the Microfil to cure completely (Appendix B).

Following the overnight curing period, the gracilis muscles from both limbs were carefully dissected and gently placed between two cover slips to maintain in situ orientation during the dehydration process. The muscles were dehydrated by a graded series of ethanol solutions (25, 50, 75, 95 and 100\%) for at least 12 hours for each alcohol dilution. Following dehydration the muscles were placed into $100 \%$ methyl salicylate for clearance (16). Once cleared, the muscle was oriented correctly, placed onto a slide, covered with permount, coversliped, and given 48 hours to dry before imaging. See Chapter 4 materials and methods for imaging. 


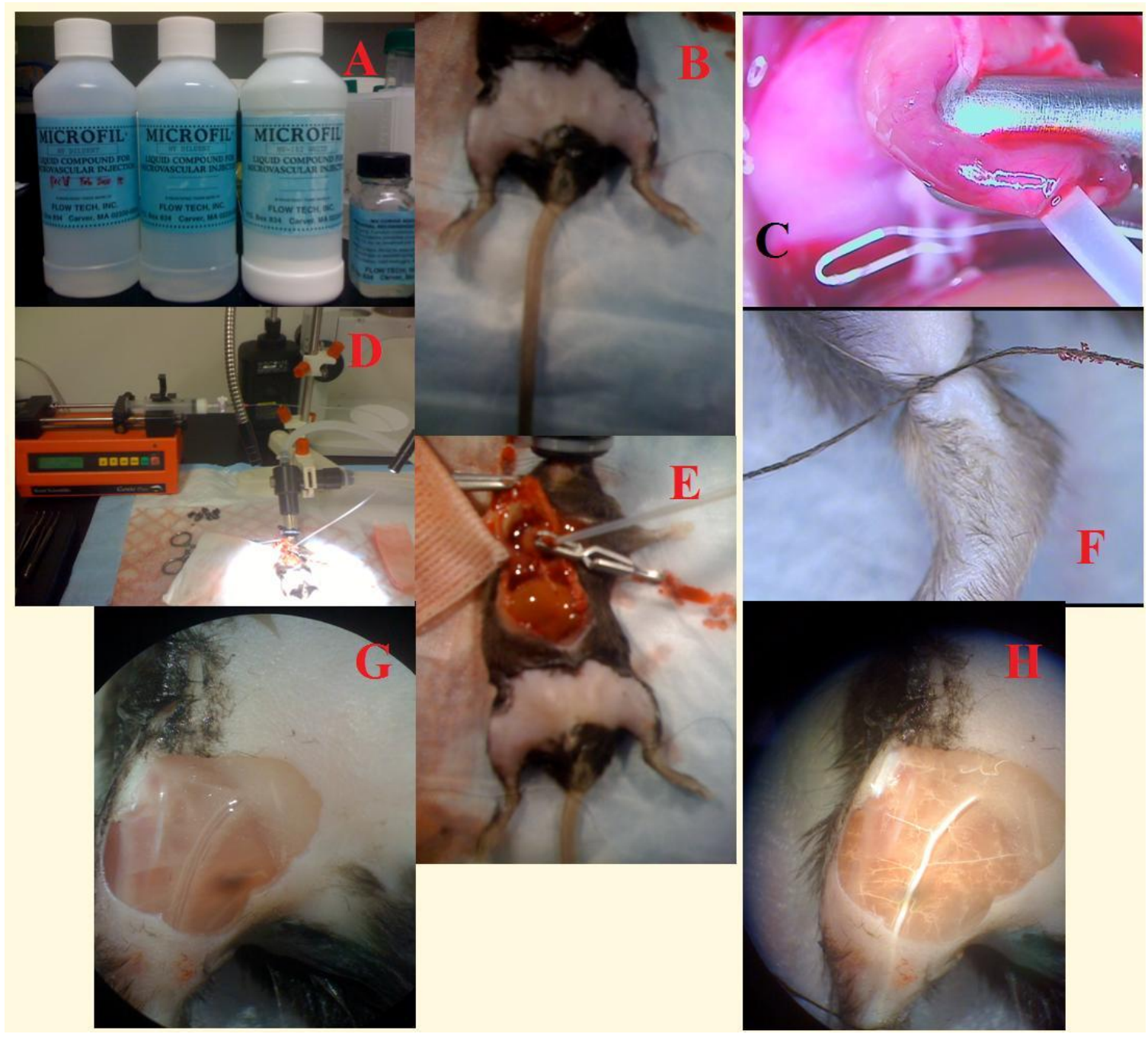

Figure 8 - A) Microfil cast ingredients (from left to right) MV-Diluent, HV-Diluent, MV-112, Curing agent. B) In order to prep the animal the hindlimbs were trimmed with clippers and then the hair was removed completely with depilatory cream to minimize hair contamination in the sample. C) An incision was cut in the apex of the heart and PE-100 catheter tubing was inserted into the apex and clamped into place with a vascular clamp. D) Following clearance the $20 \mathrm{~mL}$ syringe was loaded with the Microfil cocktail for perfusion. E) In order to gain access to the heart the ribs and diaphragm were quickly cut through and clamped back with hemostats in order to open the chest cavity. F) Ankle ligation to prevent Microfil from casting the venous side through A-V shunts. G) Proper clearance of the gracilis muscle was checked before Microfil was perfused. H) After Microfil perfusion gracilis was checked to make sure perfusion penetration was adequate and that there was no venous 


\section{Results}

Achieving the desired results required two different studies with multiple experiments. Preliminary testing of the Microfil yielded poor results (Figure 9). Further experimentation adjusting the viscosities by varying the amount of MV122 and HV-Diluent (Table 1 - Study 1) also provided poor results (Figure 10).

For study 2, the ratios of diluent and MV-122 were kept at a constant 5:4 ratio,

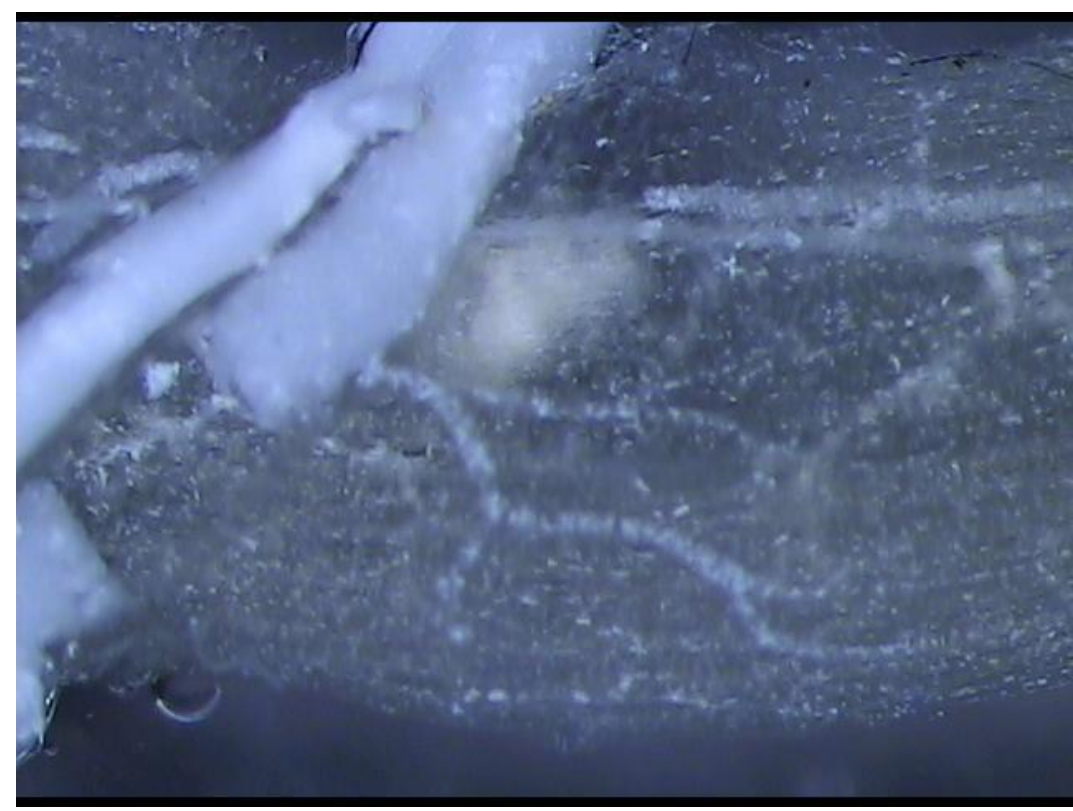

Figure 9 - Preliminary Microfil yielded poor results as the filling is incomplete and spotty. This sample is of the right anterior near the saphenous artery.

\begin{tabular}{|l|l|l|l|l|l|l|}
\hline \multirow{3}{*}{ Study 1 } & & 1 & 2 & 3 & 4 & 5 \\
\cline { 2 - 6 } & MV-122(ml) & 1.5 & 2.0 & 2.5 & 3.0 & 3.5 \\
\cline { 2 - 6 } & HV-Diluent $(\mathrm{ml})$ & 3.0 & 2.5 & 2.0 & 1.5 & 1.0 \\
\hline \multirow{3}{*}{ Study 2} & & & & & \multicolumn{2}{|c}{} \\
\hline & MV-122 (ml) & 2.0 & 2.0 & 2.0 & \multicolumn{1}{|c}{} \\
\cline { 2 - 5 } & HV-Diluent $(\mathrm{ml})$ & 2.5 & 1.25 & 0 & \multicolumn{1}{|c}{} \\
\cline { 2 - 5 } & MV-Diluent $(\mathrm{ml})$ & 0 & 1.25 & 2.5 \\
\hline
\end{tabular}

Table 1 - Studies with Microfil casting compound ratios. Study 1 was unsuccessful as adjusting the ratios between the MV-122 and Diluent ruins the compound. Study 2 was successful; it maintains the 5:4 ratio of Diluent to MV-122 while also varying the viscosity. Ultimately, experiment 2 of study 2 provided the wanted results. 
while the viscosity was adjusted by mixing MV-diluent with HV-Diluent (Table 1 Study 2). Experiment 2 provided the desired results: solid casting with no bubbles, which only penetrated to the capillary level (Figure 11).

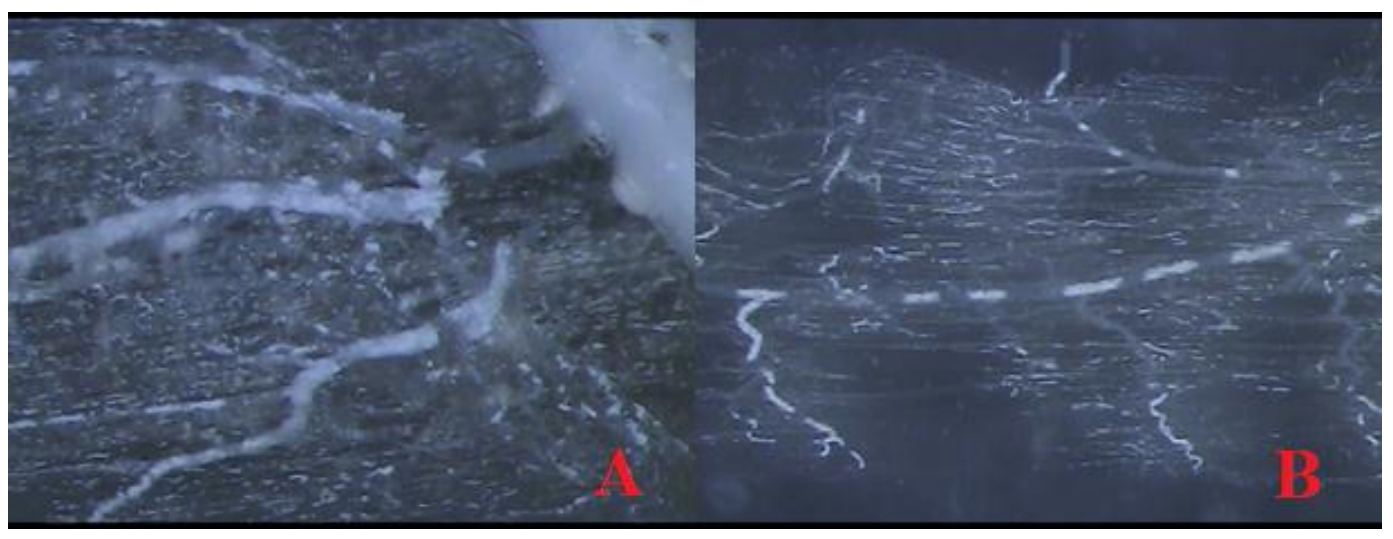

Figure $10-$ A) Poor results from the left anterior of study 1-experiment 1 near the saphenous artery. B) Poor results from the left anterior of study 1experiment 5 in the center of the gracilis muscle.

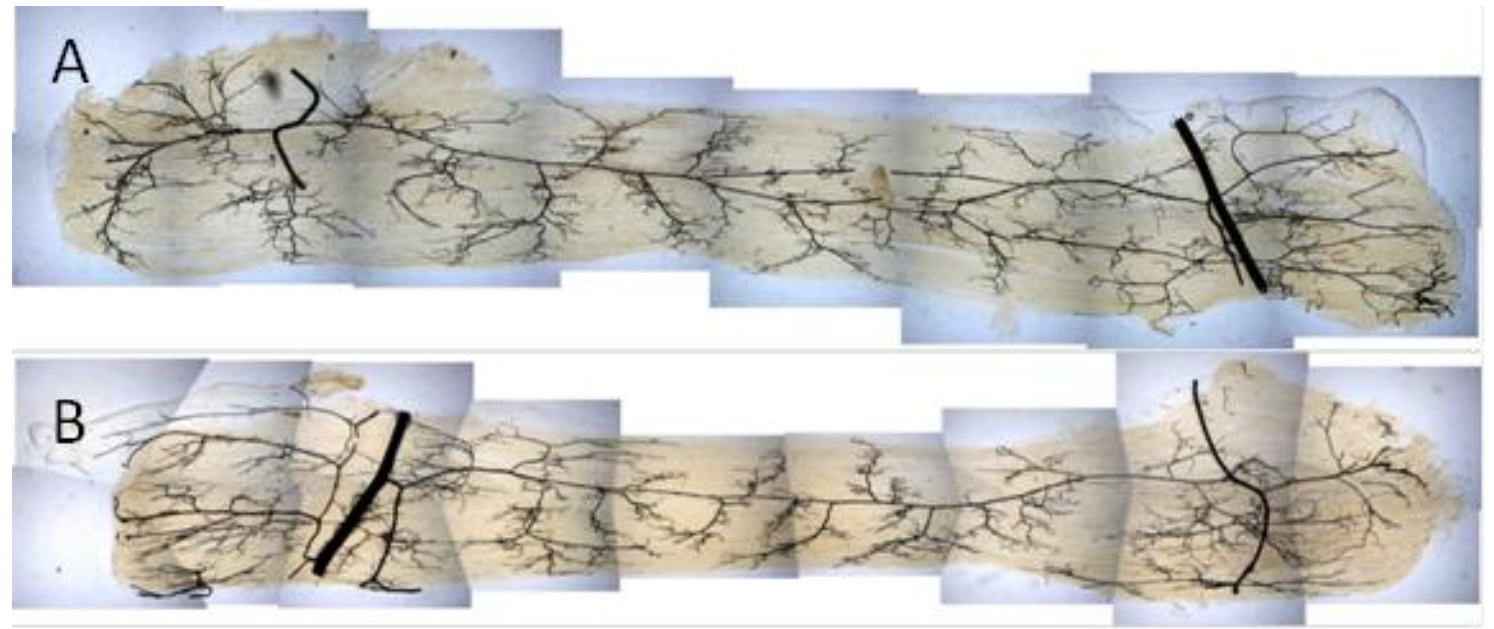

Figure 11 - Control animal B. A) Left leg anterior gracilis. B) Right leg anterior gracilis. 


\section{Discussion}

Results from the new Microfil casting procedure were a great improvement over previous India ink vascular casts. Figure 12 displays a successful India ink fill of the anterior gracilis. From Figure 12, it is clear that both the arterial and venous side of the circulation is filled. The India ink completely fills the arterial side, the capillaries (which look like strands of hair in the image) and then the

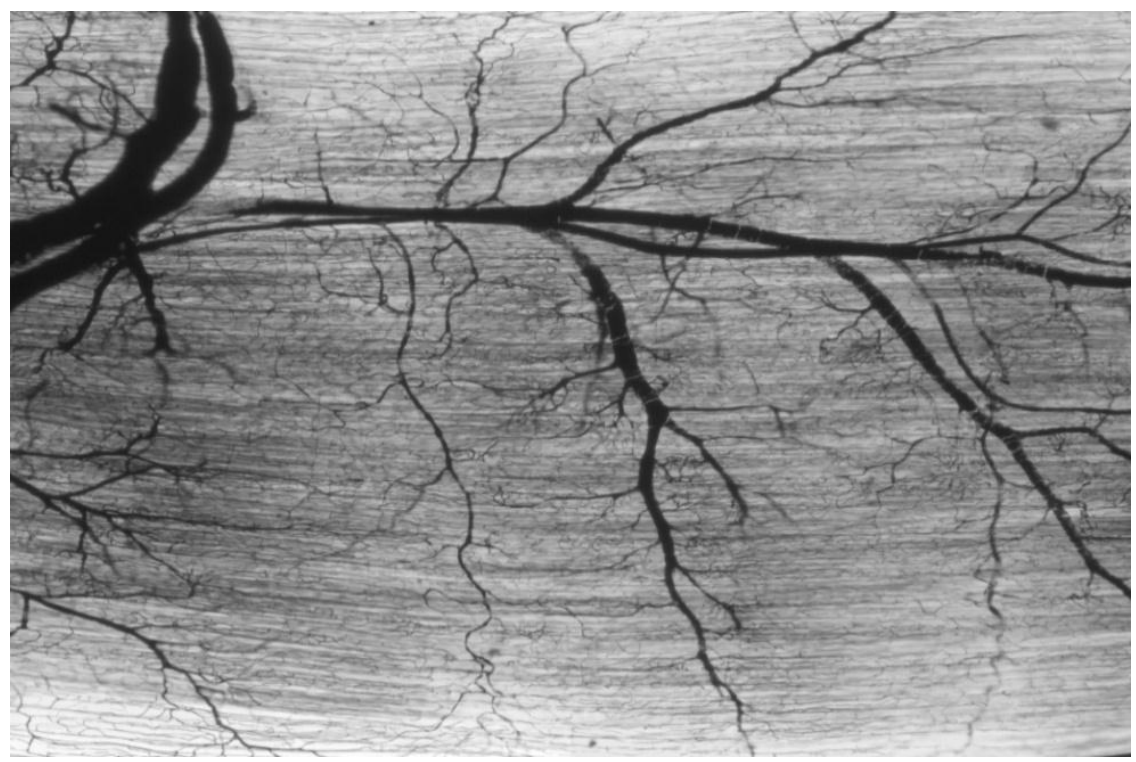

Figure 12 - A successful India ink fill casts both the arterial and venous side of the vascular system.

venous side. In order to use this sample for measurement one must visually isolate the arterial side from the venous side before proceeding. The interest for our lab lies in the arterial side of the circulation because that's where blood flow is determined and where vascular remodeling predominantly occurs following an occlusion (19); the venous side cast just adds confusion during morphology measurement. Although it is possible to discern arterial from venous by looking at 
relative size of the vessels (the venous side is larger than the arterial side) mistakes are still made and often smaller arterial trees are missed due to the complexity of the images. Arteries and veins run parallel to each other and intertwine along their paths, causing difficulties when trying to differentiate between the two. The whole process would be easier if the vascular side wasn't casted. My final Microfil vascular casts (Figure 11) show that it is possible to cast the arterial side of the anterior gracilis using a ventricular catheterization. It is clear from Figure 13 that the Microfil penetrates right up to the capillaries on the arterial side and then stops. Further, only the arterial side of the saphenous and profunda femoris is filled, which indicates that no Microfil was able to transition to the venous side through the A-V shunts in the lower leg.

As stated earlier, Microfil is a polymerizing silicone rubber compound that comes in three parts that must be mixed to create the injectable compound. This

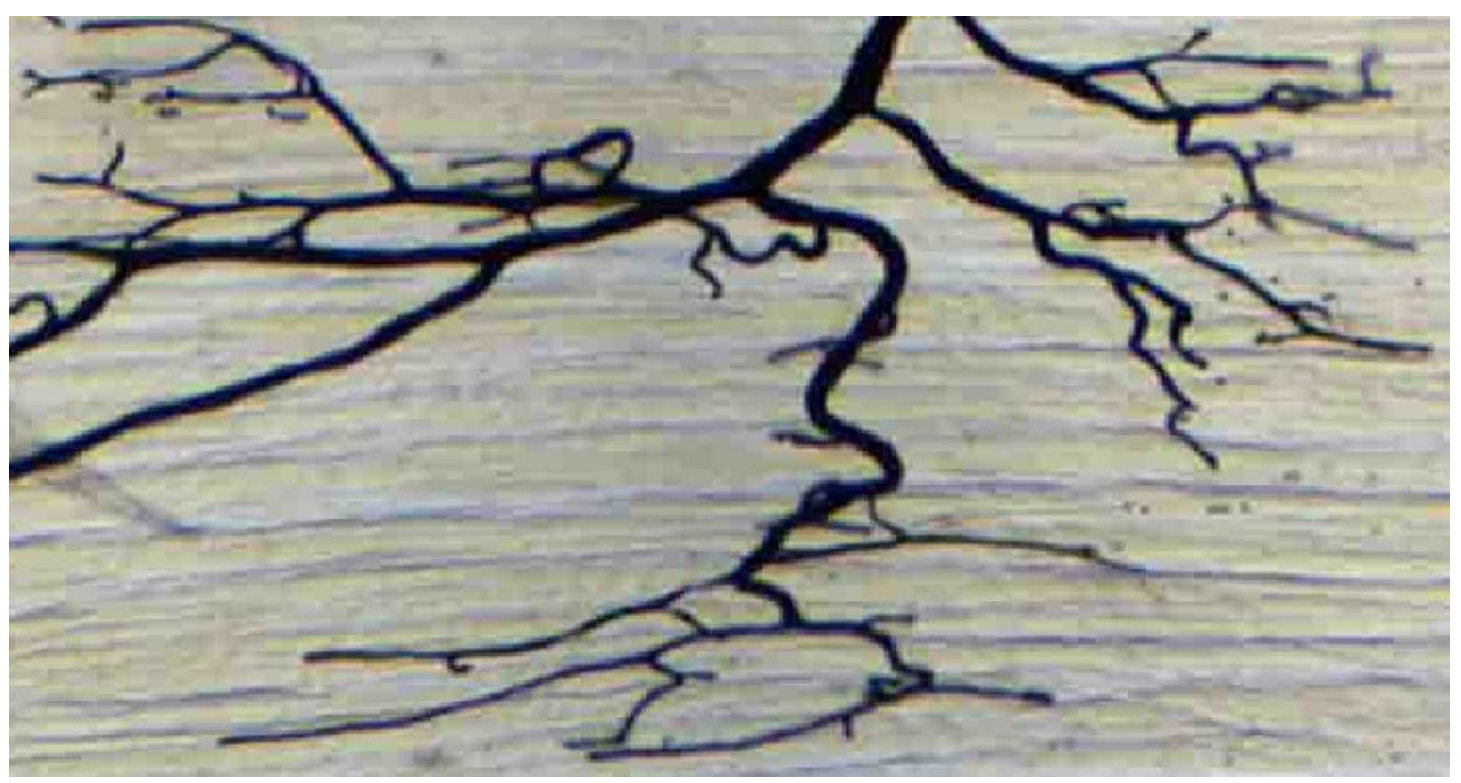

Figure 13 - Magnification of Figure 11A to show penetration of Microfil at capillary level. 
eliminates the other issues encountered with India ink as once Microfil has cured, it will not leak and the preparation involved is more straight-forward, requiring only mixing without the need to dialyze, filter, and purify (Table 2). All of the control Microfil samples can be found in Appendix A. It is important to note that

\begin{tabular}{|c|c|c|}
\hline & Microfil & India Ink \\
\hline High Contrast & Yes & Yes \\
\hline Price (16 oz) & $\$ 125$ & \$8 \\
\hline Preparation & Vortex components & Dialyze, filter and purify \\
\hline Adjustable Viscosity & Yes & No \\
\hline Curable & Yes & No \\
\hline Radiopaque & Yes & No \\
\hline
\end{tabular}

Table 2 - Tabular comparison of Microfil versus India ink although the same protocol was followed for every vascular cast, there are still variable results in the samples. This is likely due to the variability of catheter placement into the apex of the left ventricle during the ventricular catheterization. There is a very limited window of time to correctly cut the apex of the heart and clamp the catheter in place. If it is placed incorrectly it is difficult to shut off the syringe pump and reposition the catheter without damaging the tissue. There is also a risk of blood clotting, which will impede blood clearance and penetration of the casting agent into all arterial branches of the vasculature. Poor catheter position, damaged heart tissue, and blood clotting can all be factors that determine a good cast from a poor cast (Appendix A - replicate A versus replicate B).

To develop the Microfil casting protocol I began with a 4:5 ratio of Microfil MV-122 and HV-Diluent (Figure 8A), which was then catalyzed with $5 \%$ by volume of the curing agent. These values were taken from the Microfil manual 
and also used by Lash and Marxen in their research groups $(27,33)$. Using these values I began my preliminary ventricular catheterizations and the results (Figure 9) were poor, showing incomplete and spotty filling. The initial conclusion was that the poor casting was due to an error in technique with the ventricular catheterizations, so more India ink casts were performed to compare to previous results. Once correct technique was established, more Microfil casts were performed with similar results. Efforts were then focused on varying the flow rate to achieve the desired results; the idea being that if the Microfil was too viscous, increase the force of injection. The flow rate was raised to $5 \mathrm{ml} / \mathrm{min}$ (ink fills were performed at $3 \mathrm{ml} / \mathrm{min}$ ), with more poor results; any higher rate resulted in a pressure build-up that would cause the syringe pump to malfunction and sometimes catheter ejection from the heart. The next modification was to decrease the viscosity of the Microfil cocktail by adding less of the HV-Diluent and more MV-122. I experimented with a change in the HV-diluent to dye ratio in order to vary the viscosities and find a mixture that would allow for maximum penetration while also preventing venous return (Table 1); however, this approach yielded poor results, (Figure 10). The conclusion was that although the viscosities of the mixtures were different, the ideal ratio between the mixtures was changing and, therefore, the Microfil was unable to mix properly when vortexed, which yielded poor vascular casts.

A batch of the normal viscosity diluent-MV-Diluent, was ordered and used to mix with the high-viscosity diluent in a new attempt to alter the viscosity, while also still preserving the necessary ratios. This approach was markedly 
more successful, which led to discovering the best mixture for maximum arterial penetration and minimal venous return during vascular casting (Figure 11). However, although the Microfil was stopping at the capillary level, it was still not viscous enough to be stopped at the artery level and so the Microfil would perfuse down into the lower leg and across the A-V shunts. This could be seen under the microscope as the Microfil would stop at the capillaries of the gracilis but would still return on the venous side of the femoral and saphenous arteries, causing backfill into the venous circulation of the gracilis. The solution to this problem was to tie a tourniquet around the ankle of the mouse to prevent the Microfil from entering the a-v shunt (Figure 8F). This was successful, with slight modification. Initially the tourniquets were tied after the vasodilator cocktail but before the Microfil, which caused a build-up of fluid pressure in the lower leg. This pressure build-up led to a reversal of the pressure gradient when the syringe pump was stopped and caused the Microfil to be pushed back and un-fill the artery. This problem was addressed by starting the Microfil casting with no tourniquet on the ankle and applying the tourniquet a few second after the Microfil passed the tourniquet site on the ankle. At this point the Microfil flow rate had been lowered to $.5 \mathrm{ml}$ per minute. This decrease allowed for easier visualization under the microscope as well as providing ample time to perform the tourniquets.

Multiple variations of Microfil were tested before the ideal viscosity (keeping the ratio between diluent and MV-122 constant) was found. Even then, protocol adjustments needed to be made to accommodate the venous filling from 
the a-v shunts. It is also important to note that the India ink casting protocol perfused $5 \mathrm{ml}$ of ink, even though a mouse only has $\sim 2 \mathrm{ml}$ of blood volume. I had much better results when setting the pump to only deliver $1 \mathrm{ml}$ and visually inspecting the casting progress in the gracilis muscle. If the cast was complete, the Microfil perfusion was stopped. If the cast was incomplete, the pump was continued until adequate casting had been achieved. Performing vascular casts with Microfil was successful and future work in this area could test varying the ratios of the different diluents depending on the research needed. The new protocol calls for a 50/50 mixture of high-viscosity and normal viscosity diluent for the results shown. However, if further penetration of the capillaries or less penetration of the arterioles was desired, a lower or higher viscosity Microfil cocktail may be necessary, respectively. 


\section{Chapter 3 - Development of an Image Morphometry Macro Introduction}

Once the casted tissue is prepared (chapter 1) and imaged (chapter 4), morphological analysis is necessary. Important morphological parameters include: vessel length, vessel diameter, branch angle, vessel order, and vessel quantity. To manually measure these features is a laborious task; this entire project consisted of 32 samples requiring morphological analysis, with the samples averaging around 1000 vessels per sample (Appendix H). The first step in the morphological analysis is to order each vessel in each sample, followed by each morphological measurement. So, to obtain data on length, diameter, and branch angle requires 96,000 measurements, plus the time it takes to order each sample. The other issue with manual measurement is the variability in human measurement. Any type of automation or efficiency brought to this process that could decrease time and/or increase the consistency with measurement would be beneficial.

The general challenge with automatic determinations of vessel architecture is determining "vessel" from "non-vessel". An algorithm needs to be written and "trained" with numerous samples. Then the vascular system can be broken up into individual segments and functions can be written to consistently measure the different morphology.

Two areas where automatic measurement and image analysis are being pursued are ophthalmology and neuroscience-where the morphology of retinal blood vessels and neuron axons, respectively, are important $(5,8)$. Interest in 
measuring retinal vessel architecture stems from the fact that it is non-invasive and has been correlated with cardiovascular and cerebrovascular disease $(8,28)$. Retinal vessel measurement faces similar issues as our lab with manual quantification and measurements being slow and biased by the observer (8). Algorithms can be used to automatically determine arteriolar diameters (8) by fitting a vessel intensity profile to a double Gaussain function by non-linear regression, and using an edge detection algorithm. This model, created by Sobel, involves using a sliding linear regression filter to determine points of maximum intensity variation to determine "vessel" from "non-vessel" (8). Sophisticated algorithms require many samples to be tested in order to become more precise with each iteration. Chapman's group found that even their most consistent and repeatable algorithms yielded measurements significantly greater than manual measurements (8). Even though the results between automatic and manual were different, there is still value in the consistency with which an automated program can make measurements. Ideally, a full automation strategy would be designed to acquire the necessary morphology measurements, however, due to the skillset and amount of samples necessary, a different avenue from full automation was pursued.

Our interest in microvascular topology requires manual measurements of diameter, length, branch angles, vessel order, and vessel quantity for input into a computational model for calculating hemodynamic conditions. Each of these morphology measurements must be evaluated in hundreds of vessels using morphometry software, such as ImageJ. This effort requires tracing thousands of 
vessels manually, plus drawing the length and diameter of segments to obtain each of the morphological measurements; this process requires considerable time, even for those experienced with the technique-to order and measure the lengths and diameters of a sample would take about 2-3 hours, depending on the number of vessels. The goal for this portion of the research was to improve the efficiency of the manual tracing by developing a process to semi-automate the morphological analysis by combining the diameter and length measurements into one process instead of two.

\section{Materials and Methods}

\section{Design Criteria}

One of the goals of this thesis was to increase the efficiency of the morphology measurements. One of the inefficiencies I pinpointed was in the manual data collection. Ideally, a fully automated data collection program would solve this issue. However, full automation was not only outside of my skillset, but also would require approximately 100 samples to distinguish "vessel" from "notvessel"; so instead, I opted to simplify the problem significantly. The objective for this aim of the thesis was to gather two diameter measurements and two length measurements, average the diameters and lengths independently, and record both the length and diameter of a vessel when the user defined the vessel with 4 mouse clicks. This, theoretically, would save time (since the user doesn't have to measure the diameter and length separately), while also being a suitable replacement for manual measurement by outputting similar results or even 
increasing the accuracy of the data collected (since two diameter/length measurements would be averaged, instead of making one measurement).

\section{Vessel Morphometry Procedure}

The automation program was written using ImageJ and the macro editor. To reiterate, the goal for the program was to calculate the length and diameter of a vessel by drawing a quadrilateral to approximate the shape of the vessel segment (Figure 14). This is accomplished with four user-defined points, which create the quadrilateral and then output an average vessel diameter (based on the average of $\mathrm{H}_{1-3}$ and 2-4) and vessel length ( $\mathrm{L}_{1-2}$ and 3-4) (Figure 14). To define a vessel, 4 points are indicated to outline the vessel by hovering the mouse over each point and selecting the "a" key. The points must be selected in the correct order or the program will output incorrect measurements. Any mistakes can be reversed by selecting "y" to erase the previous selections. From the 4-points, the

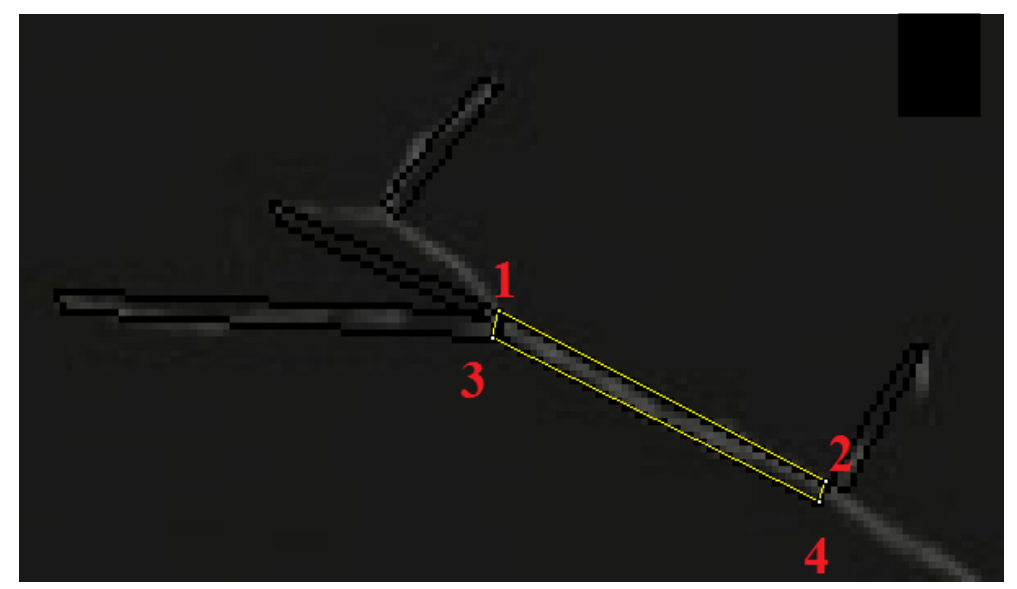

Figure 14 - General idea for how the automation program would work. The user would click the 4 points in order to identify a given vessel; the program would then draw a quadrilateral and measure the length and breadth by averaging the distance between 1-2 and 3-4 and the average distance between 1-3 and 2-4, respectively. 
macro creates a quadrilateral, whose length and height dimensions are automatically calculated and output into an ImageJ log file, which can be saved and exported into Excel (Appendix D). The code used to carry out these measurements can be found in Appendix C.

\section{Automation Validation}

As a simple test to test my macro, I drew 3 rectangles (Figure 15) and measured them manually and with the macro in ImageJ. The results (Table 3) show that the macro is calibrated (calibration is built into the macro so recalibration for every image is unnecessary-see manual protocol in Appendix L), measuring correctly (Table 3 ) and ready to be tested with a more robust
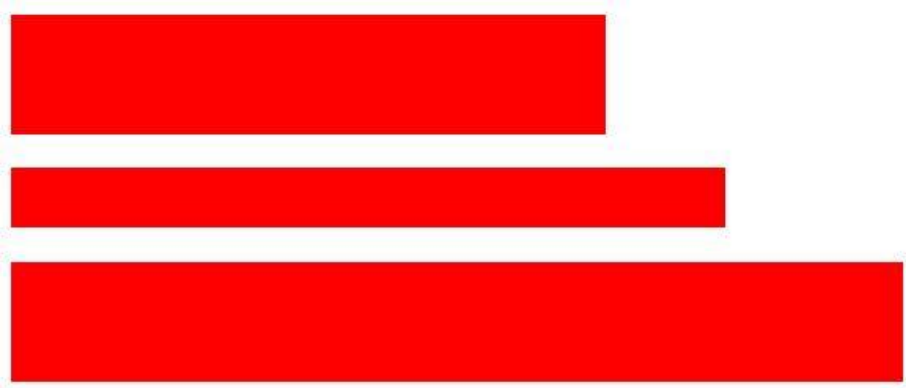

Figure $15-3$ rectangles measured manually and with the macro to test functionality.

\begin{tabular}{|l|l|l|l|l|l|l|}
\hline & \multicolumn{3}{|c|}{ Length } & \multicolumn{3}{c|}{ Diameter } \\
\hline & Manual & Macro & \% Difference & Manual & Macro & $\%$ Difference \\
\hline 1 & 655.652 & 656.522 & $0.13 \%$ & 133.913 & 131.304 & $1.95 \%$ \\
\hline 2 & 787.826 & 789.565 & $0.22 \%$ & 66.087 & 66.957 & $1.32 \%$ \\
\hline 3 & 986.087 & 986.087 & $0.00 \%$ & 133.913 & 132.174 & $1.30 \%$ \\
\hline
\end{tabular}

Table 3 - Automation validation results 
comparison on vascular casted samples. Next, four gracilis muscle samples were chosen (ECLNI, ECRNI, FCLNI and FCRNI) and were measured with the macro and manually to examine the accuracy of the program when measuring blood vessels instead of rectangles. Those four samples were chosen because they had the highest number of blood vessels, which would yield a more powerful comparison.

JMP was used for the statistical analysis to determine if the macro could substitute for manual measurement of lengths and diameters. Because there were multiple orders and therefore multiple measurements per sample a repeated measures analysis was performed; statistical significance $(p<0.05)$ between each condition was assessed using the Tukey-Kramer test, and a block was placed on the mouse to eliminate mouse-to-mouse variability within the results (Appendix E).

\section{Results}

The macro-obtained measurements were compared to the manually obtained measurements to examine if the macro could be used as an acceptable substitute to manual measurement. Average lengths and diameters from samples ECLNI, ECRNI, FCLNI and FCRNI were used to calculate the least square means for each order and then the least square means were compared for each order. The results show no significant difference between macro and manual measurement except between order 5 length values (Figure 16 and 17). 


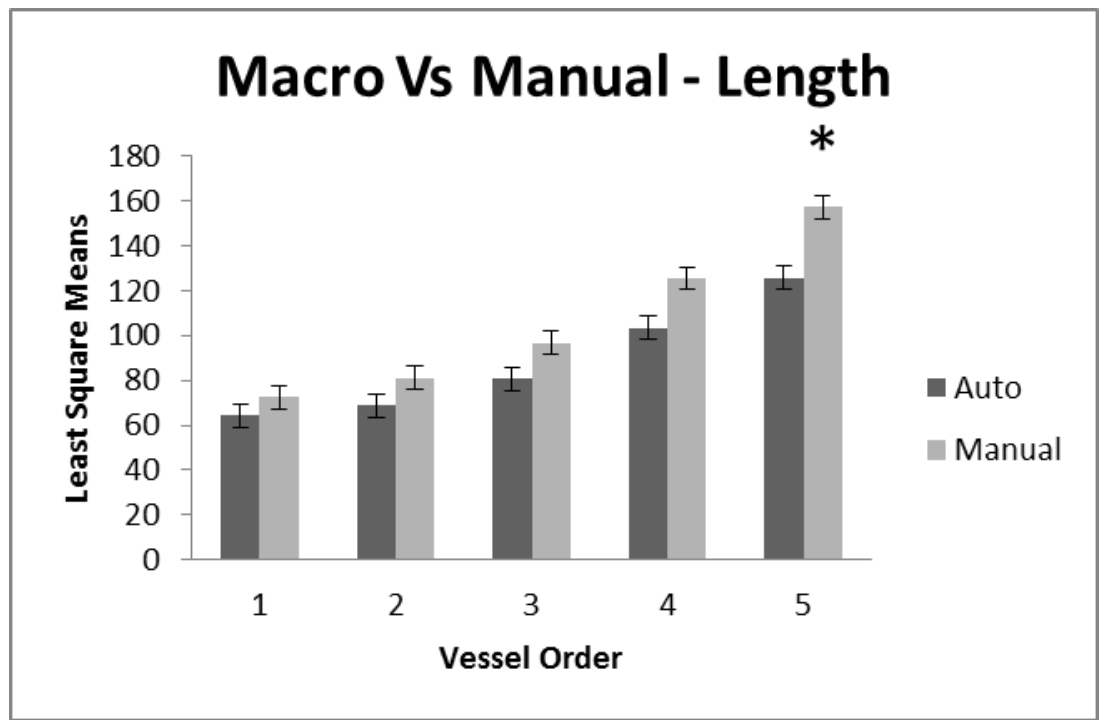

Figure 16 - Macro versus manual measurements for length showed a significant difference for order 5 vessels.

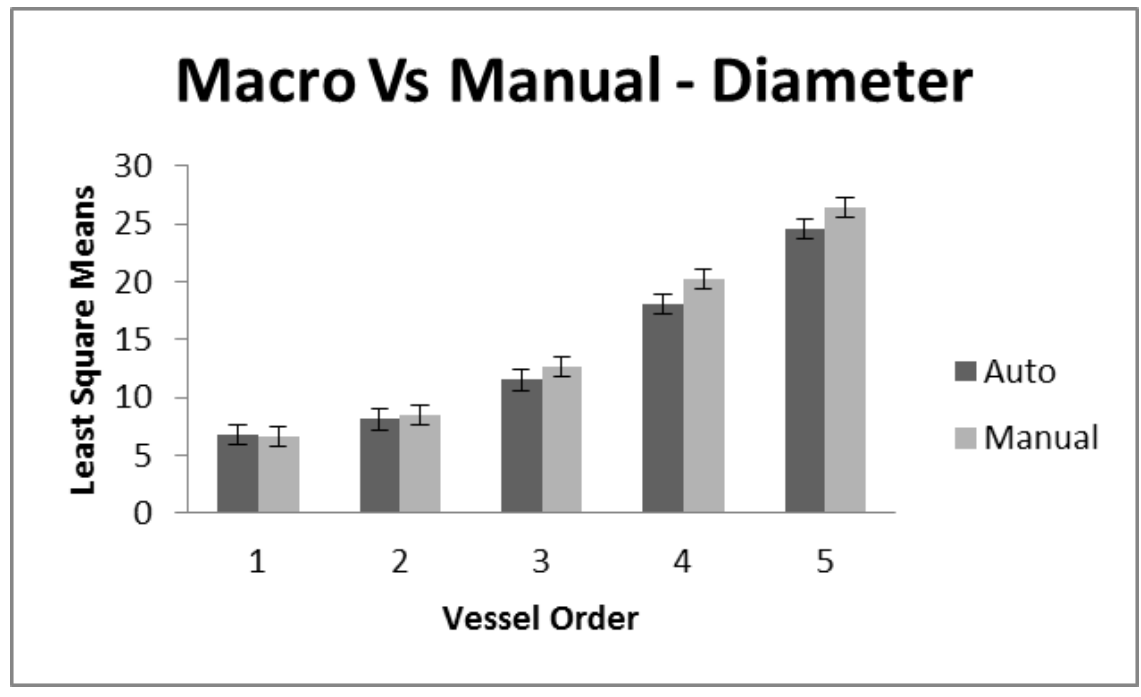

Figure 17 - Macro versus Manual measurements for diameter showed no significant differences.

\section{Discussion}

The results showed that that the only significant difference between the macro and manual measurement came from measuring order 5 lengths. The reason for this difference stems from the way that the macro is estimating values. When a vessel is straight (Figure 14) using a quadrilateral to approximate the 
vessel works well and you can expect to get an accurate value for the length of the vessel; however, when a vessel becomes more tortuous (Figure 18), a quadrilateral estimation isn't as accurate. I expected this would only be a problem with vessel lengths as the diameters are always a straight measurement. When looking at the samples it seemed as tortuosity went down the higher you went in vessel order, yet surprisingly, the only difference came in order 5 vessels, and I think the only reason for that is the sample size, which was 35 order 5 vessels. Since the program is measuring straight lines for curving vessels, the length measurements are always going to be shorter than actual measurements, but

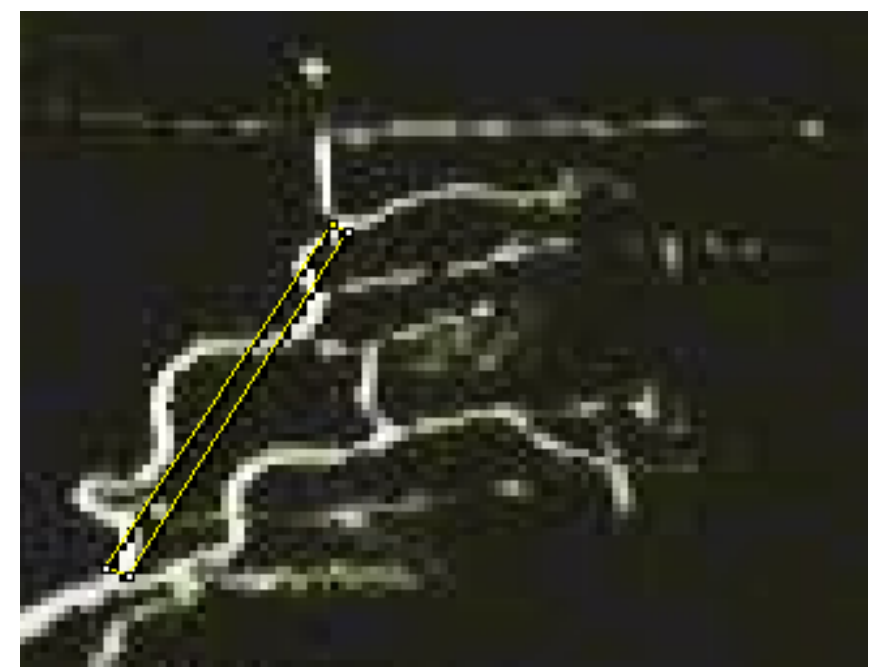

Figure 18 - Using the automation tool will approximate a vessels length if it is curved. The program output a length of $76.52 \mu \mathrm{m}$, while the manual measurement output $123.01 \mu \mathrm{m}$, which is a $37.79 \%$ difference.

statistically, this only makes a difference for order 5 vessels. Therefore, if this program is used to substitute manual measurements, order 5 lengths should still be measured manually.

The major advantage to this program is one of organization, which leads to efficiency. When four points are selected and a rectangle is drawn and 
measured, the rectangle outlining the vessel remains as a marker for which vessels have been measured already (Figure 14). This doesn't happen when using the manual measurement technique, so remembering which vessels have been measured becomes challenging. The macro also groups length and diameter into one measurement output, rather than manually measuring the length and the diameter in two different measurements.

Conversely, one difficulty with the macro is still the time it requires to make a measurement. Hovering over four different points and clicking a button on a keyboard to define thousands of vessels still takes a lot of time. The macro is a step in the right direction, but there are still improvements to be made that could save more time. Another issue is measuring the lengths of level 5 vessels. Since there are a low amount of level 5 vessels per sample an easy solution is to switch to manual measurement. Human error is another issue, since both methods require a user to define a vessel; some variance will always be present. The percent differences seen in Table 2 demonstrate that even with simple samples it's hard to define the same start and end for the manual and macro techniques; however, this small of a variance is unlikely to have any effect on the morphometry.

The initial logic for the development of the macro required the macro to wait for 4 clicks from the user, sort the points, draw the correct quadrilateral, and then make the calculations and output. However, limited programing abilities required adjusting the initial logic while still obtaining the desired output. For example, using the "getCursorLoc" command (meant to pick points when user 
clicked the left mouse button) was really hard to work with and picked up left and right clicks, click releases, and rollovers. Searching online discussion forums I came across the use of a "hot key" to label points instead of a mouse click; implementing this method eliminated the "getCursorLoc" issues, and instead of 4 mouse clicks, required the user to hover over the point of interest and press the letter "a" to define a point. Problems also arose when trying to create a "sort" function to order input selections from the user correctly, but was solved by requiring the user to select their points in the following order: upper left, upper right, lower left, and then lower right. The input always comes in the same order so the calculations are performed correctly. Output was another issue; initially the program output the values as one single column and exporting it to excel placed all of the data into cell A1 instead of individual cells. Updating the code to a new multi-column format and adding commas after each data point so that excel could use them as a delimiter allowed for excel import into separate cells, in 7 columns. Slight deviations were taken from the initial design criteria; however, the final macro accomplished the goal of recording length and diameter values with one measurement.

This program is a good starting point to progress away from manual measurement but it is still relatively basic, and numerous features could be added to optimize morphological measurement. Currently, it gathers data from four clicks to output the same length and diameter measurements as the manual method, all things being equal. Ideally, the program would gather all of the desired morphological measurements. As stated earlier, an artificial intelligence 
algorithm could solve this problem by scanning the sample and through pixel recognition decide whether a pixel is "vessel" or "not vessel". This would be done throughout the image until the program has a data-driven image of the vascular system, which it would use to compute the morphology values. This level of automation is achievable but requires intensive knowledge of algorithm development in image recognition and a high number of samples to test for algorithm augmentation. A more feasible next step for our lab would be to integrate branch angle measurement into the macro. A simple addition would use the ImageJ angle measurement tool and mark which angles have already been measured. A more elegant addition would store every defined vessel as its own element, then the user could select two adjacent elements and the program would calculate the branch angle between them. 


\section{Chapter 4 - The Impact of Collateral Enlargement on Transverse Arteriolar}

Tree Structure

\section{Introduction}

After creating a successful vascular casting protocol using Microfil and a measurement automation program, the next step was to gather morphological data on a remodeled vascular network following arterial occlusion. PAD is caused by insufficient blood flow to a tissue, which is usually induced by atherosclerotic lesions that produce increased resistance and decreased blood flow to the distal tissue, sometimes completely occluding an artery $(20,37)$. PAD has many factors that can contribute to the pathophysiology, which is why a ligated model was used to replicate an arterial occlusion in order to examine the impact collateral enlargement has on transverse arteriolar tree structure.

The goal of this model is to induce blood flow changes and repair processes that we can relate to PAD. Blood that previously flowed through the femoral and saphenous arteries will mainly be rerouted through the deep femoral and profunda femoris (34). Essentially, there are no more large arteries to feed the distal hindlimb following ligation; this causes an increase in flow as oxygen and other nutrients are rerouted through the gracilis collateral flow path to reach the lower limb. The downstream tissues experience hypoxia and inflammation in the lower leg, which promote angiogenesis (34). The ligation also causes an increase in wall shear stress through the collateral vessels from the elevated blood flow, which will result in the activation of endothelial nitric oxide synthase (eNOS) to produce NO to cause vasodilation (25). The immediate effects of the 
increased shear stress will cause vasodilation due to the blood flow divergence, while the chronic increase of shear stress will induce arteriogenesis $(4,43,44)$. Therefore, we can expect to see the collateral arteries that feed the gracilis muscle to outwardly remodel in order to provide the blood flow needed for recovery of the hindlimb $(6,7)$. The next step becomes investigating if the increased flow and shear stress in the collateral results in a change in the transverse arteriole (vessel order 4 and 5) networks branching off. This is important for a few reasons: the presence of collaterals is a decent indication of a patient with ischemic disease so stimulating arteriogenesis in these collaterals could be used as a therapy for PAD. However, it is unknown how arteriogenesis affects the microcirculation in the muscle housing the enlarged collateral and since the microcirculation is critical to nutrient and waste exchange, changes could affect muscle functionality. On one hand the increased flow could lead to architecture and vessel expansion, and on the other, the increase of pressure could lead to regression. This study could help determine if stimulating arteriogenesis could be a concern because of the possibility of compromised flow, and therefore, functionality, in the collateral-containing muscle.

\section{Materials and Methods}

\section{Ligation Surgery}

All protocols were approved by the Institutional Animal Care and Use Committee. Eight C57BL/6 mice were used as the experimental group and had a ligation tied around their left femoral artery just distal to the profunda femoris, while a sham surgery was performed on their right leg. The surgeries were 
performed under aseptic conditions and using isoflurane anesthesia. Prior to the procedure each animal was prepared by removing the hair over the surgical area with clippers and depilatory cream and then disinfecting the area with Nolvasan. The mice were maintained at $35 \pm 1.0^{\circ} \mathrm{C}$ using a rectal-temperature controlled heating pad. The initial incision was made on the medial aspect of the hindlimb, directly above the femoral neurovascular bundle, and was extended upwards to the abdominal wall and downwards towards the origin of the saphenous artery, making sure to keep the incision over the neurovascular bundle. Sterile saline solution was used to irrigate the tissue and prevent muscle desiccation. Connective tissue was blunt dissected to increase exposure. Following connective tissue removal the epigastric fat pad was removed by heat cautery to expose a site for the ligation just downstream to the profunda femoris. At the chosen spot for ligation the nerve was carefully blunt dissected away from the femoral artery, and then the vein was separated from the femoral artery in a similar fashion. To ligate, 6.0 silk suture was used; following ligation, the skin incision was closed with 7.0 polypropylene suture.

The sham surgery procedure included an incision above the femoral neurovascular bundle. Sterile saline was used to irrigate and prevent tissue desiccation while connective tissue was blunt dissected. Special care was taken not to contact the neurovascular bundle in order to simulate every aspect of the ligation except the vascular insult. Finally, the skin incision was closed with 7.0 polypropylene suture. The mouse then received a subcutaneous injection of buprenorphine $(0.075 \mathrm{mg} / \mathrm{kg})$ and was allowed to recover on a hot pad until 
ambulatory (Appendix K). Mice were then returned to the Cal Poly vivarium for 2 weeks before the vascular casting procedure.

The Cal Poly vivarium is maintained at $73 \pm 5.0^{\circ} \mathrm{F}$ and lights are on a 12 hour light-dark cycle. Each micro-isolator cage houses no more than 4 mice and is changed at least once per week. Mice are provided food and water ad libitum, in addition to the following enrichments: mouse house, nesting material, and a plastic tube. During cage changes, lab members wore isolation outfits that included: full gown, gloves, face mask, bonnet, and booties to preserve aseptic conditions. Further, mice were monitored on a daily basis, during which their health and any irregularities were recorded and/or addressed as necessary.

\section{Imaging}

Sample images were taken using a Qlmaging Retiga EXi camera at 4x magnification on an Olympus BX41 microscope using bright field microscopy (Figure 19). QCapture Pro was used to capture the images after adjusting for maximum contrast. Because of the size of the gracilis and the level of resolution needed for morphological measurements, multiple images of the sample were taken and then stitched together into a photomontage using the photomerge function in Adobe Photoshop CS3 (Appendix M). 


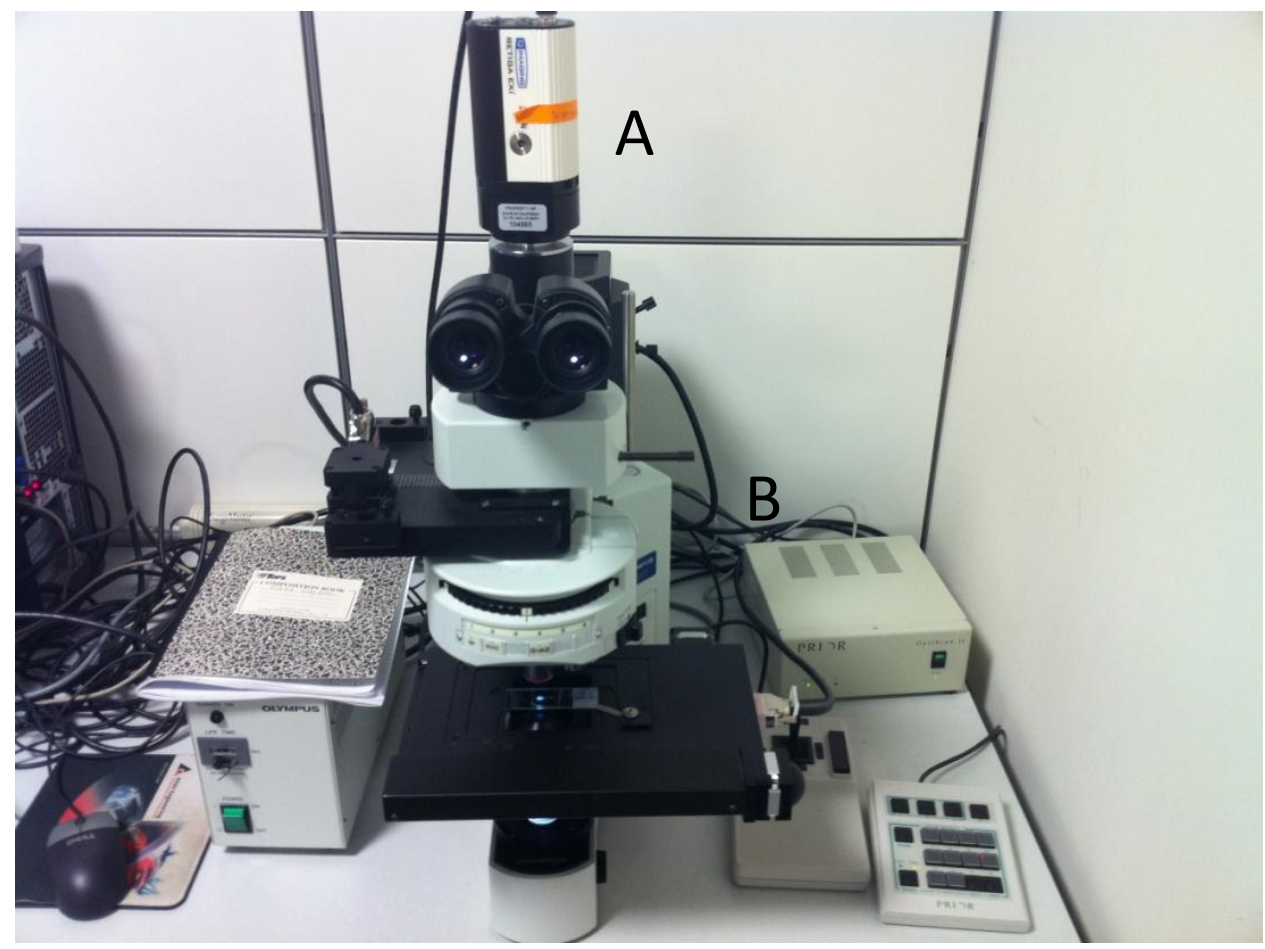

Figure 19 - Bright field microscopy setup. A) QImaging Retiga EXi camera. B) Olympus BX41 microscope.

\section{Manual Measurement using ImageJ}

Before measuring morphological parameters in a sample, branching order was determined using the Strahler method. Strahler ordering begins by defining the terminal branches of a given circulation. The terminal branches were assigned 1. When two vessels of equal order come together (i.e. order $n$ ) at a node or branch point, the parent vessel is assigned the next highest order, i.e. $\mathrm{n}+1$ (Figure 20A). When two vessels of unequal order come together at a node or branch point (i.e. order $\mathrm{n}$ and $\mathrm{n}-2$ ), the parent vessel is labeled order $\mathrm{n}$ (Figure 20B). Once all vessels were ordered morphological measurements were obtained using the macro (described in chapter 3 ) or by manual methods 
(Appendix L) and vessel counts were gathered from the total diameter measurements.

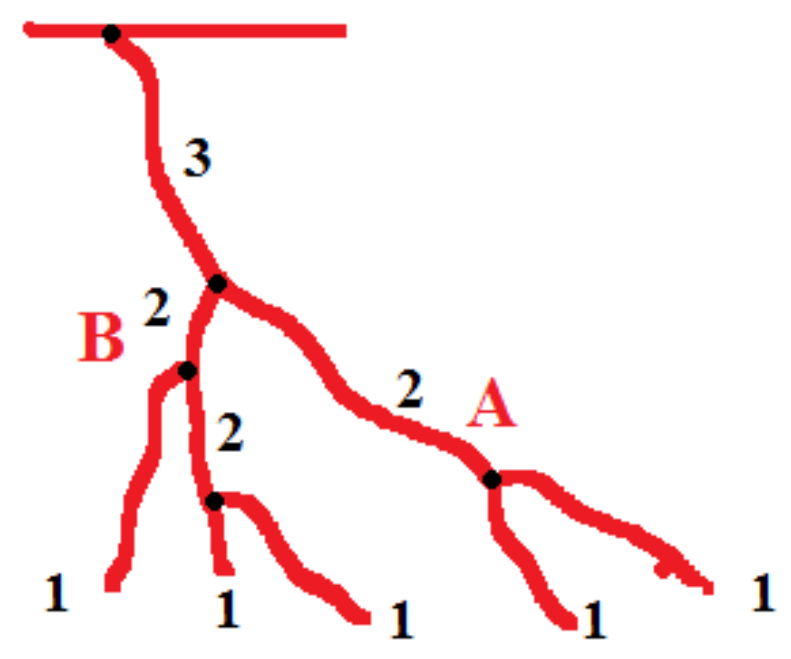

Figure $20-A$ ) When two vessels of order $n$ meet, the parent vessel is defined as order $n+1$. B) When a vessel of order $n$ and a vessel of order $n-1$ meet, the parent vessel is labeled an order $n$.

\section{Statistical Analysis}

JMP was the software packaged used for the statistical analysis. The experiment aimed to answer two questions: 1) does the sham surgery accurately reflect the true control, and 2) is there a difference in vessel morphology in the transverse arteriolar trees between the ligated and non-ligated samples? The answer to the first question determined whether the ligated limbs were compared to the sham limbs or to the true control limbs i.e. the samples from Chapter 2, where only a vascular cast was performed without surgical intervention. Before the statistical analysis, each sample was organized and measured. Each sample was ordered (Figure 20) and if a sample didn't have order 5 vessels it was labeled as a poor cast and the data was excluded from the statistical analysis. Of 
the 32 total samples 9 were labeled as poor fills. Once the sample was ordered, each ordered vessel's length and diameter was recorded and averaged so that for a given sample there was an average length and diameter for each individual order. Order 6 diameter values were taken from 5 measurements along the longitudinal arteriole, from the saphenous artery to the profunda femoris, which were then averaged. Order 6 length measurements were not taken. Because there were multiple orders and therefore multiple measurements per sample a repeated measures analysis was performed in JMP (Appendix J); statistical significance $(p<0.05)$ between each condition was assessed using the TukeyKramer test (Appendix I). When comparing sham legs to ligated legs, a block was placed on the mouse to eliminate mouse-to-mouse variability within the results. Statistical significance of vessel counts $(p<0.05)$ between the two treatments across each order was assessed using t-tests (Appendix N).

\section{Results}

From the eight ligated mice, replicates $\mathrm{C}$ and $\mathrm{H}$ were labeled as a poor casts and were excluded from analysis. From the true controls, replicates A, C and the right leg from $\mathrm{H}$ were excluded (Appendix F). Figure 21 depicts a successful vascular cast on a ligated animal. 


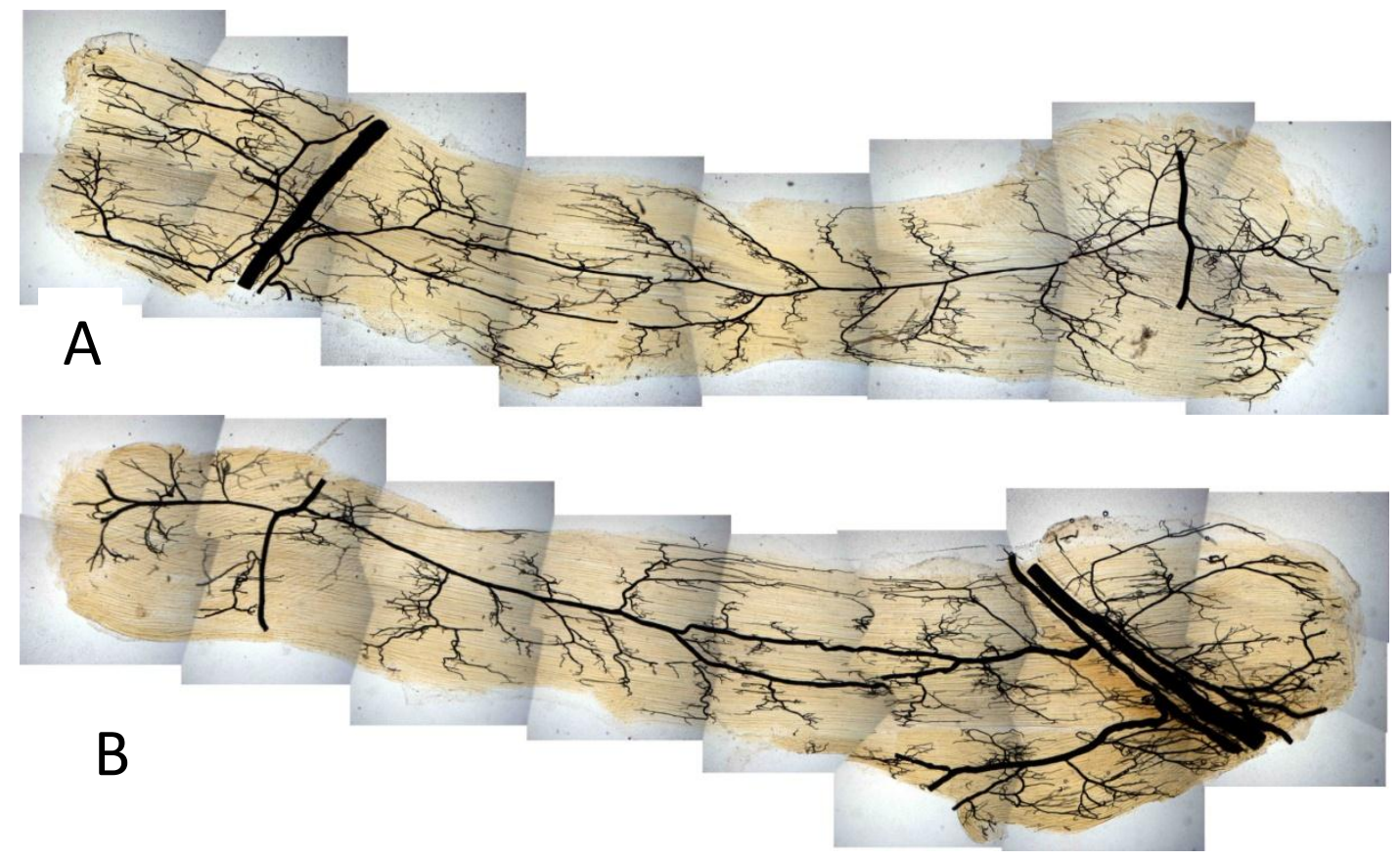

Figure 21 - Ligated animal E. A) Right leg - Sham surgery. B) Left leg - Ligated.

Average lengths and diameters from the samples (Appendix G) were used to calculate the least square means for each order. The right leg values from the ligated animals (sham) were compared to the right leg values from the animals only casted using Microfil (true control) to examine if the sham surgery was an appropriate estimation of normal. Comparing control and sham found a significant difference in the order 5 for length measurements (Figure 22A). No significant differences were found between sham and control for diameter measurements (Figure 22B). Comparing vessel counts between treatments across each order showed no significant differences (Figure 23A); there was also no significant differences between sham and ligated legs for length measurements (Figure 23B). Comparing sham and ligated legs for diameter 
measurements found a significant difference in the order 6 measurements (Figure 23C).
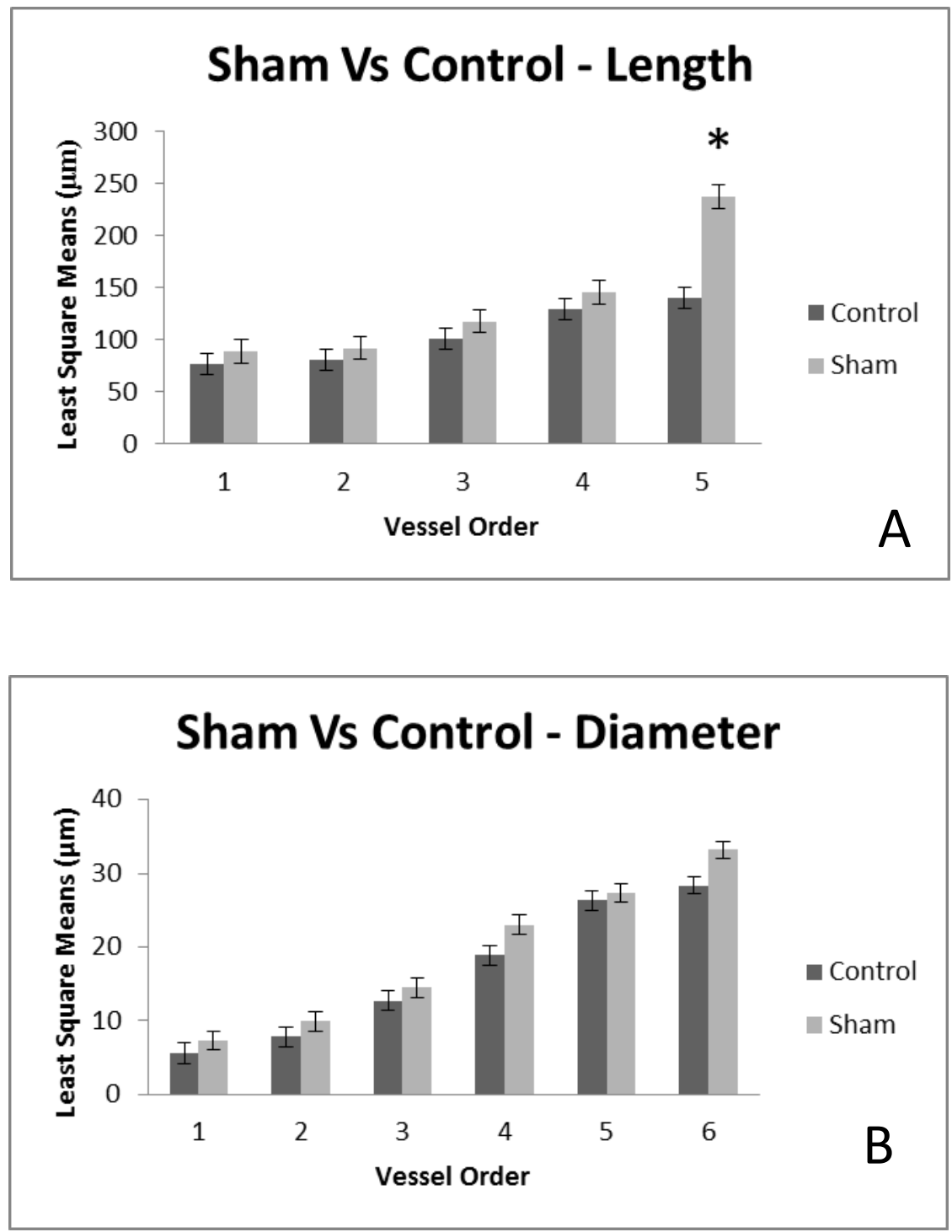

Figure 22 - Right legs (sham) of the ligated mice compared to the right legs of the control mice (Microfil only). A) Length measurements - significance found between control and sham at order 5 only. B) Diameter measurements - no significance found between any orders 

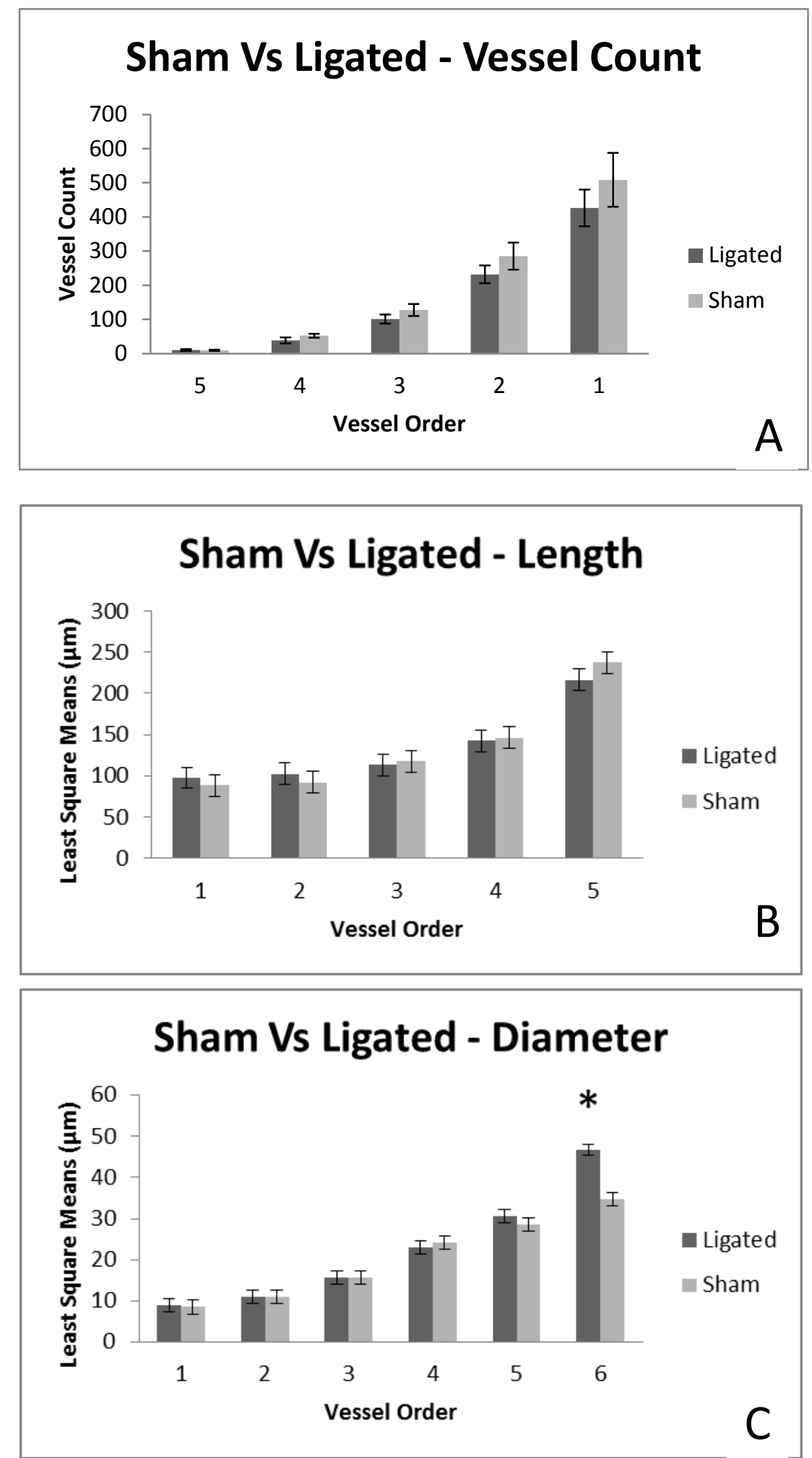

Figure 23 - Right legs of ligated mice (sham) compared to the left legs of ligated mice (ligated). A) Vessel counts - no significance found. B) Length measurements - no significance found. C) Diameter measurements - significance found between sham and ligated legs at order 6 only. 


\section{Discussion}

Many factors contribute to the pathophysiology of PAD (46); therefore, a mouse model was used to model one aspect of the pathology: an arterial occlusion. The occlusion offered an increase in blood flow to the collateral circuit in order to examine whether arteriogenesis impacts the transverse arteriole architecture branching from the collateral. This could allow a better understanding into how therapies to stimulate arteriogenesis would impact the microvasculature and tissue function.

Comparing the sham surgery versus the right leg of the true controls yielded a significant difference between the lengths of the order 5 vessels, with the sham lengths being significantly greater than the control lengths. Although we see this difference in the order 5 lengths of the sham and control samples, this alone is not enough to say that the sham surgery cannot be used to estimate the true control. Poiseuille's law states that, all else being equal, an increase in length will result in a decrease in volumetric flow. However, the length is not as powerful of a factor as the radius of the vessel and there aren't any significant differences in the order 5 or even the order 6 diameters. This difference in length is likely not a reflection of differences in the underlying biology, but rather a reflection of insufficient replicates and therefore the sham can be used as a control and simply opening the animal for surgery will not cause any significant vessel remodeling for our purposes.

Comparing the sham leg to the ligated leg yielded a significant difference between the diameters of the order 6 vessels. To reiterate, the order 6 vessels 
refer to the collateral arteriole that runs from the profunda femoris to the saphenous artery. Arteriogenesis in this vessel is well-described (16), and is induced by the elevated flow (shear stress) that is resultant of an increased pressure gradient across the collateral vessel following ligation. Increased sheer stress is a trigger for increases in arterial diameter and outward remodeling of the arterial wall will occur to normalize the hemodynamic shear stress $(4,19,38)$. So, one reason why the elevated flow didn't alter transverse arteriolar architecture is because the increased hemodynamic shear stress caused by the ligation had dissipated by the time the transverse arteriolar level was reached due to the arteriogenesis seen in the collaterals. Another explanation could be due to cellular communication between gap junctions that override the hemodynamic stimuli and cause the network to remain relatively unchanged. Therefore, it's possible that stimulating arteriogenesis in a clinical environment should not cause any negative effects in the collateral-containing muscle. However, this observation is potentially limited by the differences between the ligation model and a patient.

The femoral artery ligation model is a simplified model of a patient with PAD (it only simulates an occlusion), which is why it's important to understand the differences between the model and PAD. One of the main differences is the cause for the conditions, which are significantly different. When the femoral artery is ligated, the arterial occlusion occurs quickly, as the suture is pulled taut. In the case of a patient with PAD, occlusion occurs as a result of atherosclerosis, which occurs gradually as the build-up of plaque causes the narrowing of the 
arteries over many years (46), however, when a thrombus forms, partial occlusion transitions to complete occlusion rapidly. In patients with PAD, complete occlusion of arteries often results in limb necrosis without proper treatment $(10-40 \%$ have primary amputations and there is a mortality rate of 40 $70 \%$ in 5 years) (14). Further, humans with PAD typically present with other comorbidities like heart disease or obesity and have a gradual arterial narrowing due to atherosclerosis at multiple vascular sites throughout the body (46). It is likely that these co-morbidities explain the lack of revascularization. This results in a slower impairment of blood flow and progression of hypoxia and could alter the mechanisms for vascular repair (46). Conversely, in the mouse, hindlimb revascularization occurs and the mouse is able to recover over a period of 2-4 weeks sans intervention or treatment (34). The ligation model results in an arterial occlusion at one point, so, while both PAD and the model induce an occlusion in the extremities and hindlimb, respectively, the mechanisms are different. It is important to be aware of these differences as one interprets experimental results and makes conclusions regarding the process of revascularization and how it may influence recovery of vascular function following arterial occlusion when using a mouse model.

Interesting future experiments could gather branch angle measurements to add to the analysis. Further, flow measurements could be taken for the whole muscle to examine the impact of architecture versus reactivity or experiments could examine the impact architecture has on vasodilation. Future applications of this research could try to address one of the major concerns with therapeutic 
angiogenesis: tumor-like microcirculation. Previous experiments delivering basic FGF or VEGF developed stable vascular networks; however, they soon began to resemble immature tumor vasculature $(11,14)$. It's important to understand why that happened by better understanding the network repair process. This study examined the repair process in a simple animal model, so the next step would either be to examine different muscles in a hypoxic area or to deliver growth factors and see how the network changes, which could eventually lead to finding what morphology values differentiate a healthy network from a tumor network and how a network can be prevented from diverging from stable to tumor-like. Other experiments would examine the role of gap junction communication in network remodeling by performing similar experiments in connexin knockout mice.

Before proceeding with future experiments it would be advantageous to investigate new methods to make data collection more efficient. Measuring the samples requires a high level of resolution for order 1 vessels; 15-25 photomicrographs must be captured and then montaged, sometimes manually. Ideally, a high-resolution camera would capture a full sample image with enough detail that measurement of order 1 vessels is possible. High resolution cameras capable of this task exist but are available in the $\$ 40,000$ range. A more plausible solution would be to capture up-close representative images. This would require identification of the large transverse arteriole trees that reach order 5 vessels; these arteriole trees would be ordered and measured and would give a sufficient representation of the vascular network. Of the samples prepared there were 
between two and five of these arteriole trees per sample, on average. Capturing only the large transverse trees would decrease the images needed from between 15 and 25 for an entire sample to between 5 and 15 to capture the chosen trees. 


\section{Chapter 5 - Discussion}

Previous studies have revealed the complexity of vascular remodeling (19, $31,38)$ and relatively simple treatments in clinical trials have yielded less than optimal results $(11,14)$. In order for therapeutic angiogenesis to be successful, there needs to be a more comprehensive understanding of the vascular growth process to understand why therapeutic angiogenesis was unsuccessful in clinical trials. A more complete understanding of the effects of revascularization on vascular network topology could provide information to devise more efficacious therapeutic angiogenesis treatments for patients with PAD.

The overall objectives of this study were to establish procedures to increase the efficiency of microvascular network morphology and use these procedures to investigate microvascular remodeling following arteriogenesis. The aims for the thesis were as follows:

1) Create a vascular casting protocol to only cast the arterial side of the microcirculation.

2) Decrease the time to measure microvascular morphology through some form of automation.

3) Determine the impact of collateral artery enlargement on the topology of arteriolar networks that branch from the collateral artery.

Through Aim 1, I developed a vascular casting protocol to only cast the arterial side of the microcirculation with the commercially available casting compound Microfil. This protocol required an adjustment of the Microfil viscosity. The ideal mixture for casting the arterial side was a ratio of $X M V-122$ to $Y$ of 
both the high and normal viscosity diluent (as long as Microfil was prevented from entering the lower leg $A-V$ shunt). Because this was successful, the venous side no longer complicated the image; this allowed for a more thorough vessel identification of arterial trees, trees that were previously lost among the confusion of India ink casting were now measurable. When ordering an India ink cast it is difficult to discern between arteries and veins, especially for lower ordered vessels. This leaves the user to select arterial trees that were easy to identify. This results in much lower vessel numbers than the Microfil casting technique. Since Microfil casting makes it much easier to identify arterial vessels, more vessels were measured (Appendix $\mathbf{H}$ ) and, therefore, more time was required for data collection. Future modifications could order the entire sample and then only measure the arteriole trees that reach order 5 before connecting back to the longitudinal arteriole. This aim only examined the gracilis, but future work could adapt the protocol to include other muscles like the spinotrapezius, and it could even be adjusted to fill less of the arterial circulation, depending on the branching order of interest.

Through Aim 2 I decreased the time for morphological measurement of the anterior gracilis with a macro written in ImageJ. For this procedure, the vessel is defined with four clicks; the macro creates a quadrilateral with the points and then measures the two lengths and diameter values to output an average length and diameter for the selected vessel. Conversely, manual measurement requires separate length and diameter measurements. Surprisingly, results found that the macro's estimation of length was only significantly different for order 5 vessels, 
where the sample size was small. Overall, the macro is a first step in making morphometry measurements more efficient. The next iteration of the process would add a vessel angle measurement to the macro, such that all three measurements are bundled into one step. One of the unforeseen side effects of the new vascular casting protocol was that because the venous vasculature was un-casted, arterial vasculature was much easier to identify, which increased vessel numbers significantly and therefore inadvertently increased the measurement time. To maintain efficiency, focus should be kept on measurement of well-casted vascular trees; meaning, after ordering a sample, measurements should only be made in vascular trees that reach order 5 before connecting back to the longitudinal arteriole.

For the $3^{\text {rd }}$ and final aim, I used the casting procedure and morphology macro, developed in Aims 1 \& 2, to compare transverse arteriolar networks branching from an enlarged collateral to transverse arteriolar networks branching from a non-enlarged collateral. As expected the collateral arteriole (order 6) did outwardly remodel. We expected that the increased flow and shear stress in the collateral would impact the transverse arteriole networks branching from the collateral. Surprisingly, there was no significant difference for any diameters, lengths, or vessel numbers for orders 1-5, indicating that the networks aren't significantly changing. This could be due to the lack of hemodynamic shear stress (absorbed by the outward remodeling of the collateral arteries), which would cause outward remodeling or it could be due to cell-to-cell communication through gap junctions overriding the hemodynamic stimuli. 
It is important to note that using Microfil as a casting agent doesn't completely eliminate issues arising with discerning vessels for measurement. Complex vessel branching patterns necessitate subjective measurement of multidirectional networks that frequently overlap and intertwine. The gracilis muscle is also three-dimensional, so any vessels in the z-direction may not be measured accurately, however, since the gracilis is relatively flat, this likely didn't impact on results. Another issue identifying vessels stems from inconsistent dissecting of the gracilis muscle that leaves some samples with other muscles that underlie the gracilis and add layers of vessels that are unwanted.

Overall, pairing the new Microfil casting technique with the photomerge stitching functionality in Photoshop yields an image that shows the larger picture of vascular structure following an arterial occlusion. Furthermore, because of the high resolution of the images, one is able to make measurements of lower order vessels to gather data to explore changes in the vascular structure. The ligation model experiments demonstrated functionality of the new techniques and laid the groundwork for further experiments to be performed where vascular topology is examined rather than vessel cross-sections for the development of PAD treatments. Ultimately, more thorough analysis of microvascular network topology following arterial occlusion should assist in the development of successful therapeutic angiogenesis treatments for patients with PAD. 


\section{References}

1. Baumgartner I, Pieczek A, Manor O, Blair R, Kearney M, Walsh K, and Isner JM. Constitutive expression of phVEGF165 after intramuscular gene transfer promotes collateral vessel development in patients with critical limb ischemia. Circulation 97: 1114-1123, 1998.

\section{Berliner JA, Navab M, Fogelman AM, Frank JS, Demer LL, Edwards} PA, Watson AD, and Lusis AJ. Atherosclerosis: basic mechanisms. Oxidation, inflammation, and genetics. Circulation 91: 2488-2496, 1995.

3. Buschmann I and Schaper W. Arteriogenesis Versus Angiogenesis: Two Mechanisms of Vessel Growth. News Physiol Sci 14: 121-125, 1999.

4. Cai W and Schaper W. Mechanisms of arteriogenesis. Acta Biochim Biophys Sin (Shanghai) 40: 681-692, 2008.

\section{Cardona A, Saalfeld S, Arganda I, Pereanu W, Schindelin J, and} Hartenstein V. Identifying neuronal lineages of Drosophila by sequence analysis of axon tracts. J Neurosci 30: 7538-7553.

\section{Chalothorn D, Moore SM, Zhang H, Sunnarborg SW, Lee DC, and} Faber JE. Heparin-binding epidermal growth factor-like growth factor, collateral vessel development, and angiogenesis in skeletal muscle ischemia. Arterioscler Thromb Vasc Biol 25: 1884-1890, 2005.

7. Chalothorn D, Zhang H, Clayton JA, Thomas SA, and Faber JE. Catecholamines augment collateral vessel growth and angiogenesis in hindlimb ischemia. Am J Physiol Heart Circ Physiol 289: H947-H959, 2005.

\section{Chapman N, Witt N, Gao X, Bharath AA, Stanton AV, Thom SA, and} Hughes AD. Computer algorithms for the automated measurement of retinal arteriolar diameters. Br J Ophthalmol 85: 74-79, 2001.

9. Chung MK and Rich MW. Introduction to the cardiovascular system: http://www.niaaa.nih.gov/Resources/GraphicsGallery/CardiovascularSystem/Publishing Images/270f1.gif, 1990.

10. Dejana E. Endothelial cell-cell junctions: happy together. Nat Rev Mol Cell Biol 5: 261-270, 2004.

11. Dellian M, Witwer BP, Salehi HA, Yuan F, and Jain RK. Quantitation and physiological characterization of angiogenic vessels in mice: effect of basic fibroblast growth factor, vascular endothelial growth factor/vascular permeability factor, and host microenvironment. Am J Pathol 149: 59-71, 1996. 
12. Djonov V and Makanya AN. New insights into intussusceptive angiogenesis. EXS: 17-33, 2005.

13. Djonov VG, Kurz H, and Burri PH. Optimality in the developing vascular system: branching remodeling by means of intussusception as an efficient adaptation mechanism. Dev Dyn 224: 391-402, 2002.

14. Dormandy J, Heeck L, and Vig S. The fate of patients with critical leg ischemia. Semin Vasc Surg 12: 142-147, 1999.

15. Folkman J. Angiogenesis: an organizing principle for drug discovery? Nat Rev Drug Discov 6: 273-286, 2007.

16. Gruionu G, Hoying JB, Pries AR, and Secomb TW. Structural remodeling of mouse gracilis artery after chronic alteration in blood supply. Am J Physiol Heart Circ Physiol 288: H2047-2054, 2005.

17. Guyton AC and Hall JE. Textbook of Medical Physiology. Philadelphia: Elsevier Saunders, 2006.

18. Heil M, Eitenmuller I, Schmitz-Rixen T, and Schaper W. Arteriogenesis versus angiogenesis: similarities and differences. J Cell Mol Med 10: 45-55, 2006.

19. Helisch A and Schaper W. Arteriogenesis: the development and growth of collateral arteries. Microcirculation 10: 83-97, 2003.

20. Hirsch AT, Criqui MH, Treat-Jacobson D, Regensteiner JG, Creager MA, Olin JW, Krook SH, Hunninghake DB, Comerota AJ, Walsh ME, McDermott MM, and Hiatt WR. Peripheral arterial disease detection, awareness, and treatment in primary care. JAMA 286: 1317-1324, 2001.

21. Institute NHLaB. Anatomy of the Heart:

http://www.nhlbi.nih.gov/health//dci/images/heart interior.gif.

22. Institute NHLaB. Normal Artery and Artery With Plaque Buildup:

http://www.nhlbi.nih.gov/health/public/heart/pad/images/about/pad il flow plaque.g if.

23. Isner JM. Myocardial gene therapy. Nature 415: 234-239, 2002.

24. Jain RK. Molecular regulation of vessel maturation. Nat Med 9: 685-693, 2003.

25. Kelsall CJ, Brown MD, and Hudlicka O. Alterations in reactivity of small arterioles in rat skeletal muscle as a result of chronic ischaemia. Journal of Vascular Research 38: 212-218, 2001. 
26. Kelsall CJ, Brown MD, Kent J, Kloehn M, and Hudlicka O. Arteriolar endothelial dysfunction is restored in ischaemic muscles by chronic electrical stimulation. J Vasc Res 41: 241-251, 2004.

27. Lash JM and Bohlen HG. Functional adaptations of rat skeletal muscle arterioles to aerobic exercise training. J Appl Physiol 72: 2052-2062, 1992.

28. Liew G, Wang JJ, Mitchell P, and Wong TY. Retinal vascular imaging: a new tool in microvascular disease research. Circ Cardiovasc Imaging 1: 156-161, 2008.

29. Losordo DW and Dimmeler S. Therapeutic angiogenesis and vasculogenesis for ischemic disease - Part I: Angiogenic cytokines. Circulation 109: 2487-2491, 2004.

30. Losordo DW and Dimmeler S. Therapeutic angiogenesis and vasculogenesis for ischemic disease - Part II: Cell-based therapies. Circulation 109: 2692-2697, 2004.

31. Makanya AN, Hlushchuk R, and Djonov VG. Intussusceptive angiogenesis and its role in vascular morphogenesis, patterning, and remodeling. Angiogenesis 12: 113-123, 2009.

32. Marieb EN. Human Anatomy \& Physiology: Benjamin Cummings, 2003.

33. Marxen M, Sled JG, and Henkelman RM. Volume ordering for analysis and modeling of vascular systems. Ann Biomed Eng 37: 542-551, 2009.

34. Mees B, Wagner S, Ninci E, Tribulova S, Martin S, van Haperen R, Kostin S, Heil M, de Crom R, and Schaper W. Endothelial nitric oxide synthase activity is essential for vasodilation during blood flow recovery but not for arteriogenesis. Arterioscler Thromb Vasc Biol 27: 1926-1933, 2007.

35. Michel CC and Curry FE. Microvascular permeability. Physiol Rev 79: 703-761, 1999.

36. Nagy JA, Vasile E, Feng D, Sundberg C, Brown LF, Manseau EJ, Dvorak AM, and Dvorak HF. VEGF-A induces angiogenesis, arteriogenesis, lymphangiogenesis, and vascular malformations. Cold Spring Harb Symp Quant Biol 67: 227-237, 2002.

37. Ouriel K. Peripheral arterial disease. Lancet 358: 1257-1264, 2001.

38. Peirce SM and Skalak TC. Microvascular remodeling: a complex continuum spanning angiogenesis to arteriogenesis. Microcirculation 10: 99-111, 2003. 
39. Richardson TP, Peters MC, Ennett AB, and Mooney DJ. Polymeric system for dual growth factor delivery. Nat Biotechnol 19: 1029-1034, 2001.

40. Risau W. Mechanisms of angiogenesis. Nature 386: 671-674, 1997.

41. Ross R. Mechanisms of atherosclerosis--a review. Adv Nephrol Necker Hosp 19: 79-86, 1990.

42. Schaper J, Borgers $\mathbf{M}$, and Schaper W. Ultrastructure of ischemiainduced changes in the precapillary anastomotic network of the heart. $A m \mathrm{~J}$ Cardiol 29: 851-859, 1972.

43. Scholz D, Ito W, Fleming I, DeindI E, Sauer A, Wiesnet M, Busse R, Schaper J, and Schaper W. Ultrastructure and molecular histology of rabbit hind-limb collateral artery growth (arteriogenesis). Virchows Arch 436: 257-270, 2000.

44. Scholz D, Ziegelhoeffer T, Helisch A, Wagner S, Friedrich C, Podzuweit T, and Schaper W. Contribution of arteriogenesis and angiogenesis to postocclusive hindlimb perfusion in mice. J Mol Cell Cardiol 34: 775-787, 2002.

45. Semenza GL. HIF-1 and mechanisms of hypoxia sensing. Curr Opin Cell Biol 13: 167-171, 2001.

46. Shamoun F, Sural N, and Abela G. Peripheral artery disease: therapeutic advances. Expert Rev Cardiovasc Ther 6: 539-553, 2008.

47. Tsai MC, Chen L, Zhou J, Tang Z, Hsu TF, Wang Y, Shih YT, Peng HH, Wang N, Guan Y, Chien S, and Chiu JJ. Shear stress induces synthetic-tocontractile phenotypic modulation in smooth muscle cells via peroxisome proliferator-activated receptor alpha/delta activations by prostacyclin released by sheared endothelial cells. Circ Res 105: 471-480, 2009.

48. Vincent KA, Shyu KG, Luo Y, Magner M, Tio RA, Jiang C, Goldberg MA, Akita GY, Gregory RJ, and Isner JM. Angiogenesis is induced in a rabbit model of hindlimb ischemia by naked DNA encoding an HIF-1alpha/VP16 hybrid transcription factor. Circulation 102: 2255-2261, 2000.

49. Wahlberg E. Angiogenesis and arteriogenesis in limb ischemia. J Vasc Surg 38: 198-203, 2003.

50. Waters RE, Terjung RL, Peters KG, and Annex BH. Preclinical models of human peripheral arterial occlusive disease: implications for investigation of therapeutic agents. J Appl Physiol 97: 773-780, 2004. 
51. Zakrzewicz A, Secomb TW, and Pries AR. Angioadaptation: keeping the vascular system in shape. News Physiol Sci 17: 197-201, 2002. 


\section{Appendix A}

The following are the true control samples. ACRNI is a full sample label where "AC" denotes control animal a, "R" denotes right leg, and "NI" denotes nonischemic.

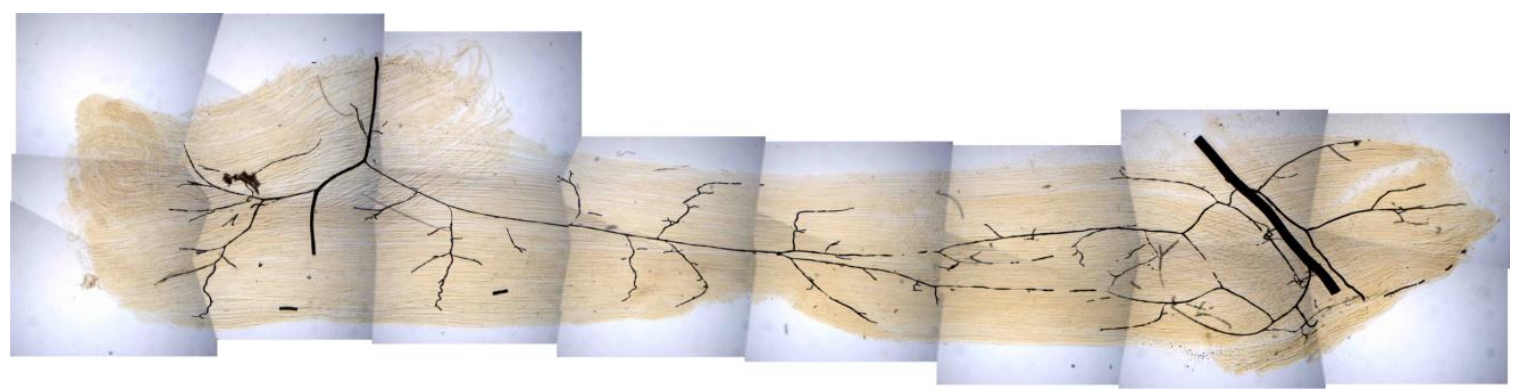

ACLNI

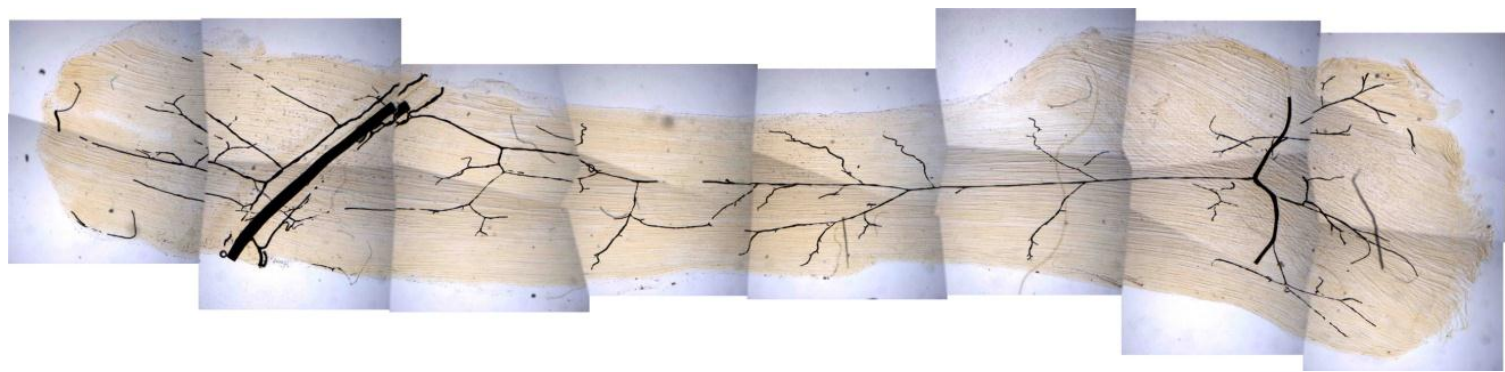

ACRNI

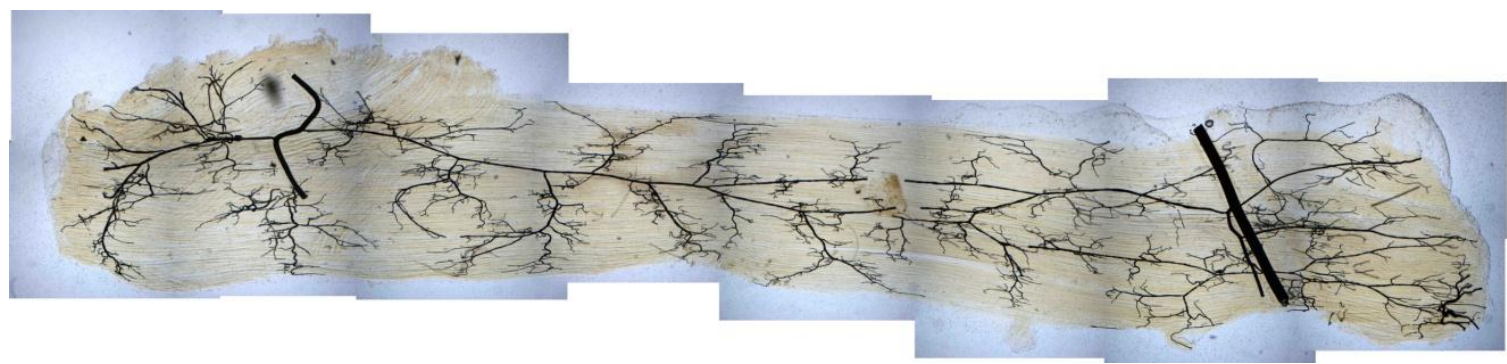

BCLNI

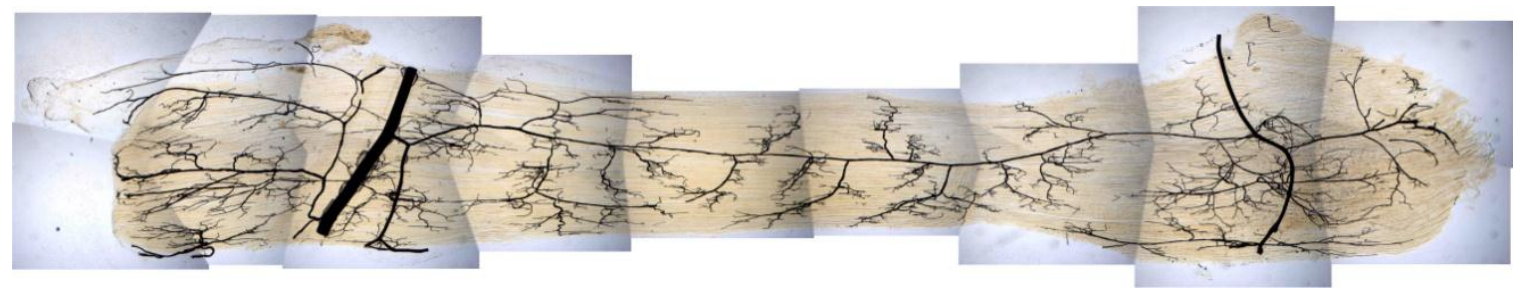

BCRNI 

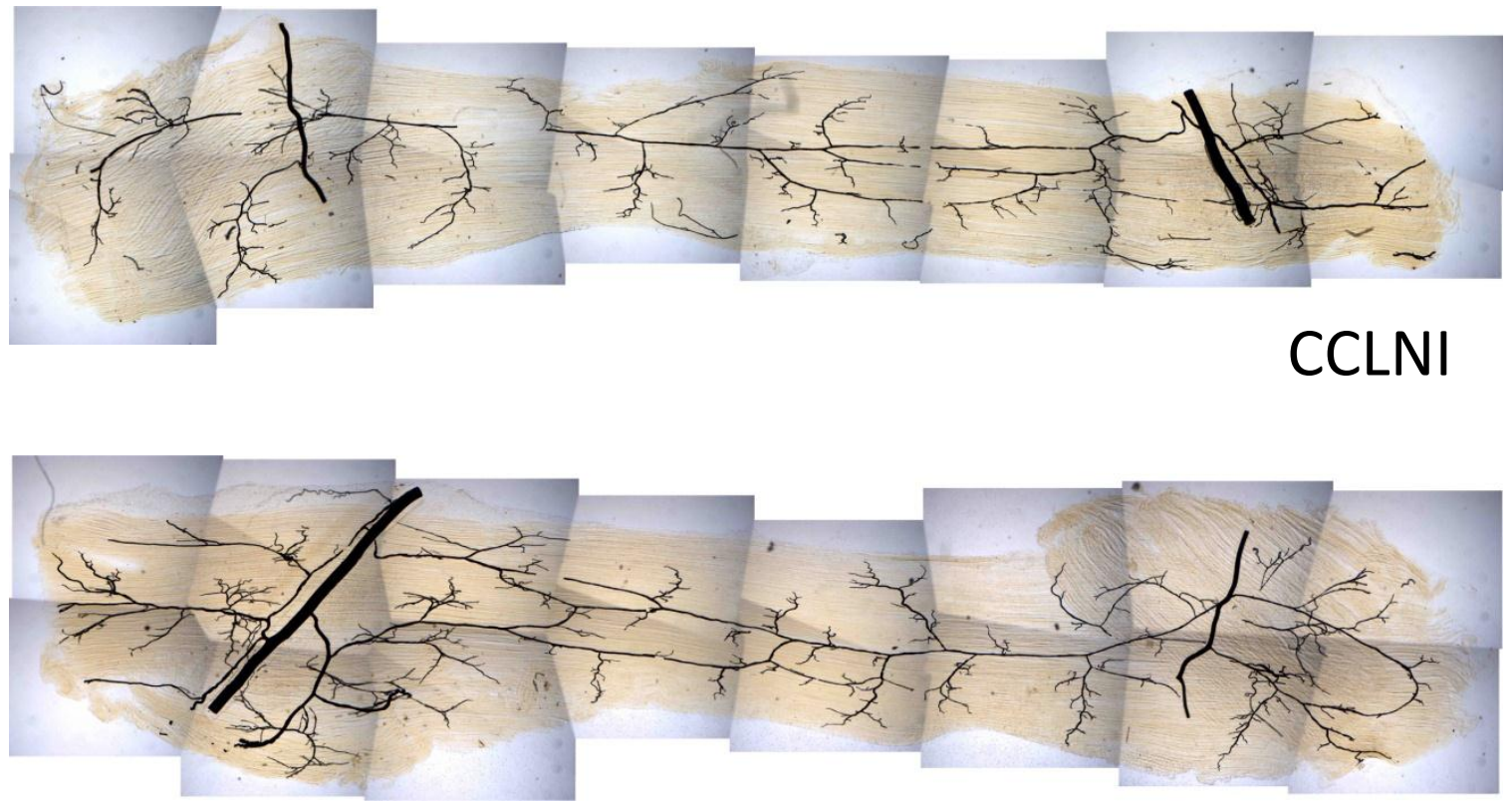

CCRNI
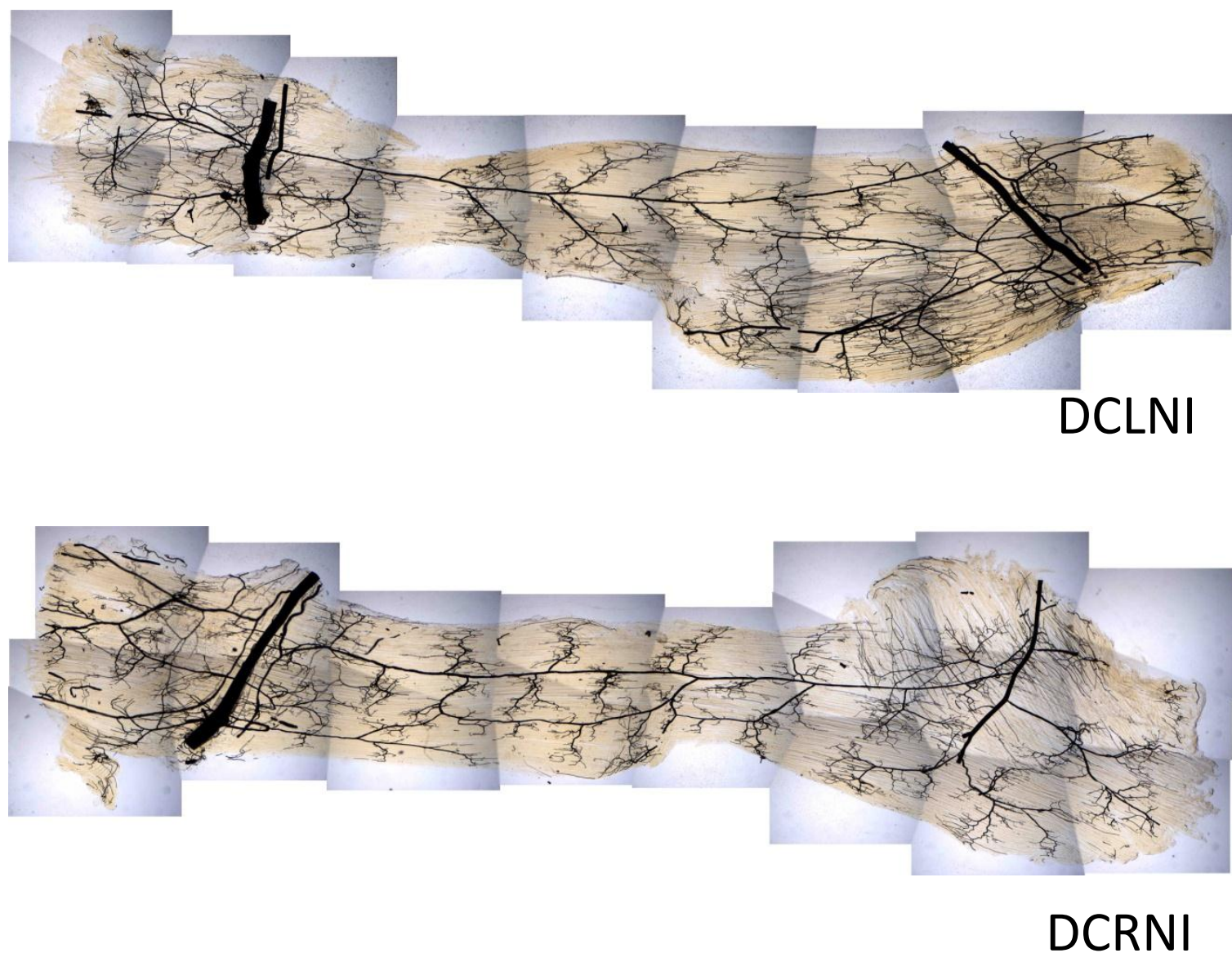

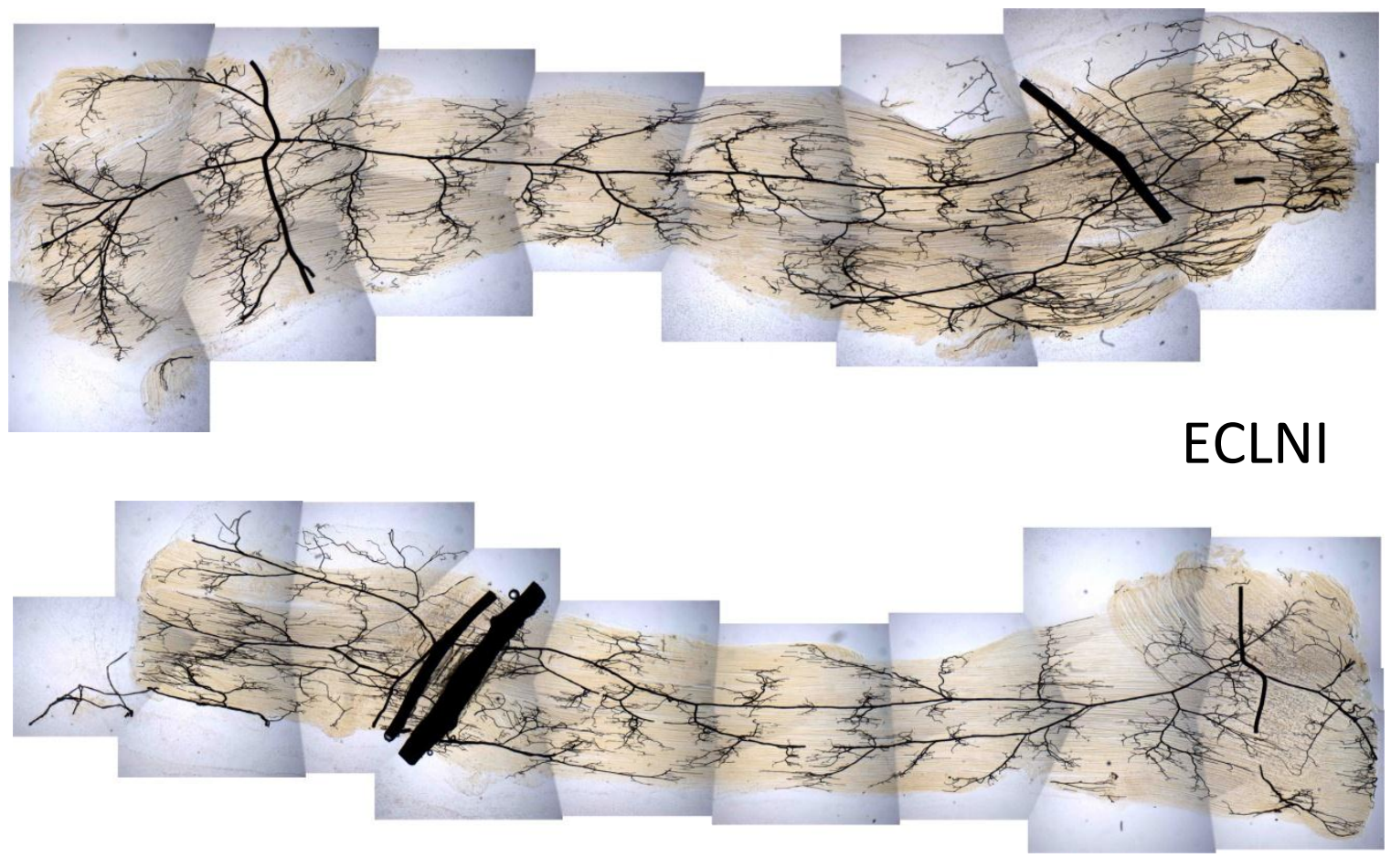

ECRNI
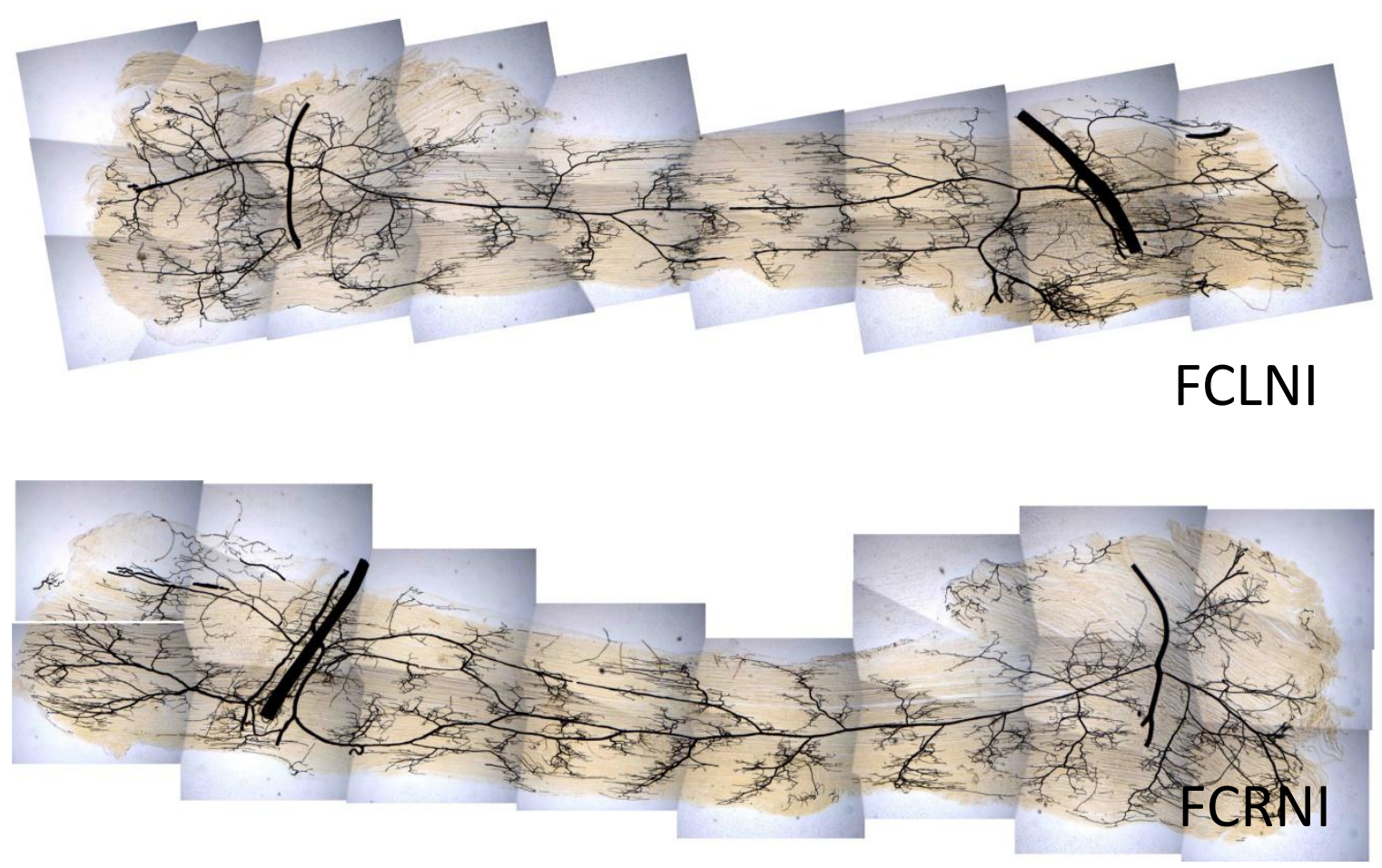

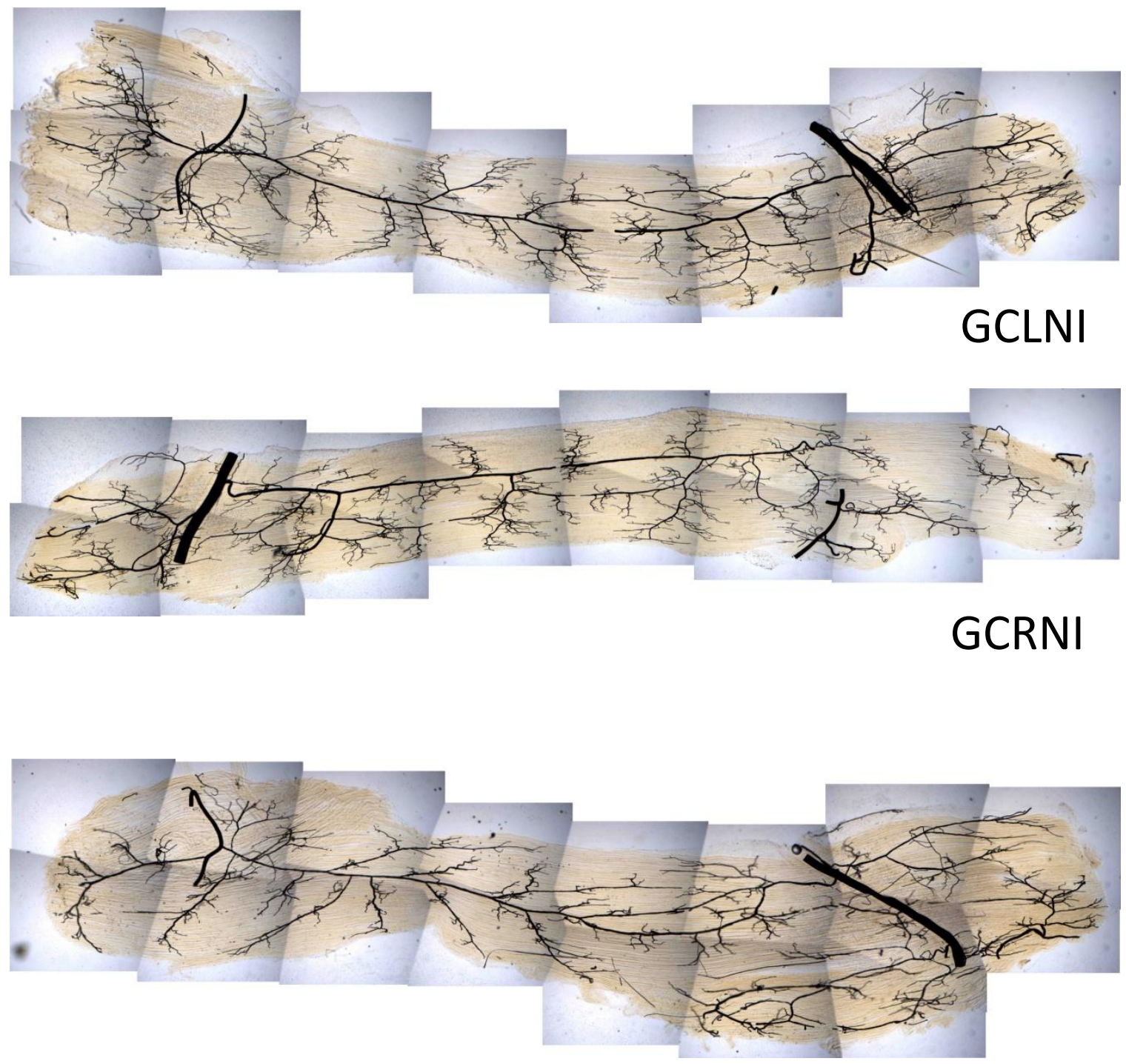

\section{HCLNI}

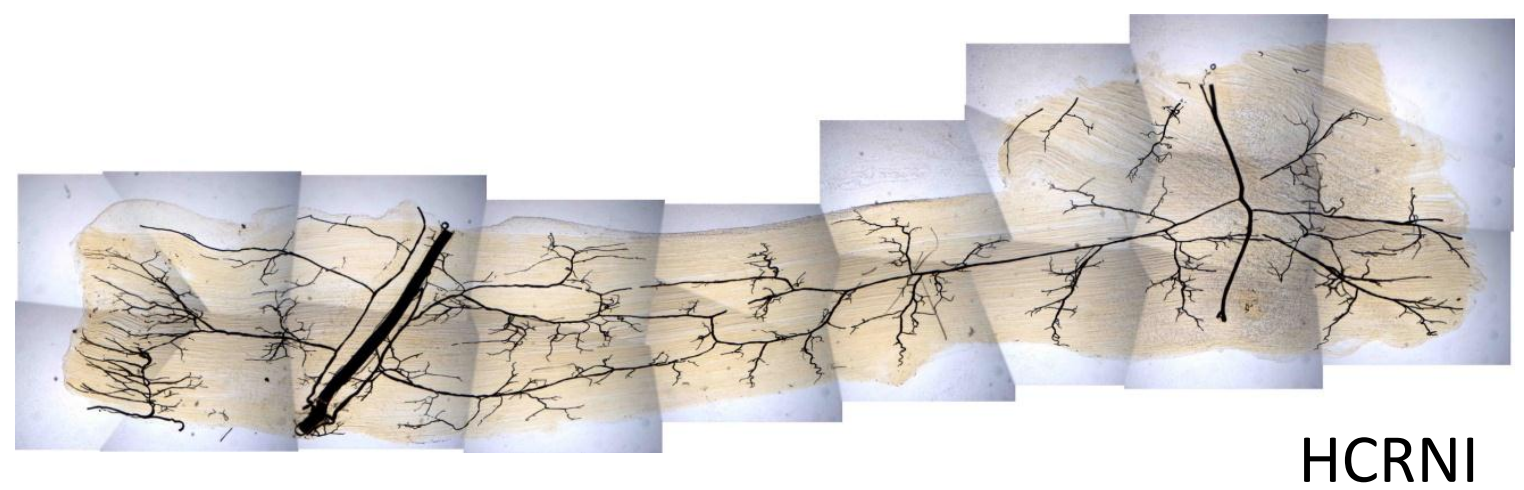




\section{Appendix B - Microfil casting and dissection protocol}

Date

Mouse Information

DOB:

Sex:

Tag:

Genotype/strain:

Cage:

Weight (g):

\section{Materials}

Non-Sterilize Dis sectionInstruments

1. Forceps (1)

2. Bone Scissors(1)

3. Skin Scissors (1)

4. Hemostats (1)

5. Spring Sciss ors for Ventricle (1)

6. Vascular clamp (1)

Obtained in surgery suite

$\begin{array}{ll}\text { 7. } & \text { Tape } \\ \text { 8. } & \text { 20mL Syringe (1) } \\ \text { 9. } & \text { Bench cover } \\ \text { 10. } & \text { Heating pad } \\ \text { 11. } & \text { Catheter/Stopcock/Blunt needle (PE-100) } \\ \text { 12. } & \text { Is oflurane Anesthetic } \\ \text { 13. } & \text { Gauze squares }\end{array}$

Vasodilator Cocktail Preparation

14. Turn on water bath to $37^{\circ} \mathrm{C}$

15. $400 \mu \mathrm{L}$ heparin

16. $1 \mathrm{~mL}$ SNP (orange)

17. $600 \mu \mathrm{L}$ Adenosine (clear)

18. $38 \mathrm{~mL}$ PBS solution

19. Thaw SNP and Adenosine

20. Add heparin, SNP, Adenosine, and PBS solution together in a $50 \mathrm{~mL}$ conical

21. Place vas odilator cocktail in water bath

Microfil Cocktail Preparation

22. 2mL Microfil MV- 122

23. $1.25 \mathrm{~mL}$ Micro fil HV-Diluent

24. $1.25 \mathrm{~mL}$ Microfil MV-Diluent

25. Add MV-122, HV-Diluent \& MV-Diluent into a $14 \mathrm{~mL}$ conical, vortex, place in bath

\section{Procedure Preparation}

26. Weigh animal

27. Obtain saline filled petri-dish, cotton swab, and instruments

Fixation

28. Anesthize mouse with Is oflurane

29. Heat up heat pad in microwave and wrap with bench cover when warm

30. Remove hindlimb hair on both legs by shaving

31. Tape animal down to heated bench cover

32. Separate skin from muscle from the abdomen to the top of the thoracic cavity

33. Fill $20 \mathrm{~mL}$ syringe with warm Vaso D
Microfil

34. Cut through abdomen close to diaphragm

35. Quickly cut through the ribs and diaphragm to open chest cavity and clamp back with hemostats

36. Cut away excess tis sue around the heart

37. Make a small incision in the apex of the heart

38. Insert catheter and clamp with vascular clamp and cut right atria

39. Inject Vaso D solution into animal approximately $20 \mathrm{~mL}$ at $5 \mathrm{~mL} / \mathrm{min} \times 2$

40. Add $.2 \mathrm{~mL}$ of curing agent to Microfil

41. Inject $1 \mathrm{~mL}$ of Microfil Cocktail (. $5 \mathrm{~mL} / \mathrm{min})$

42. Pause microfilm and ligate both ankles with silk suture once Microfil has passed the knee. Resume Microfil.

43. Repeat step 41 until satisfied with fill.

44. Remove mouse from heat pad and take out catheter (keep heart clamped)

45. Place mouse in bag (cover open wounds with saran wrap) and let sit overnight at room temp

46. Cover scope, turn off water bath, turn off oxygen, turn off Is oflurane, clean instruments

Dissection Date:

Non-Sterilized Dis section Instruments

47. Fine Forceps (2)

48. Forceps (1)

49. Iris Scissors (1)

50. Dissection scissors (1)

Obtained in surgery suite

51. Non-sterile saline

52. Cotton swab

53. Bench cover

54. Tape

55. Petri Dishes (2)

56. Cover Slips (2)

57. Disposable pipettes (1)

58. $25 \%$ ethanol

59. Dissect chosen muscles and place on cover slip and then in labeled petri dish of $25 \%$ ethanol

60. Cover scope and clean instruments

Notes:

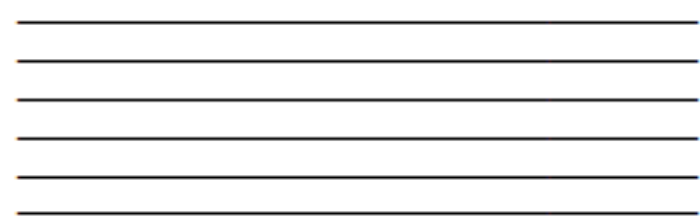




\section{Appendix C - Program code - Logic comments are displayed in green}

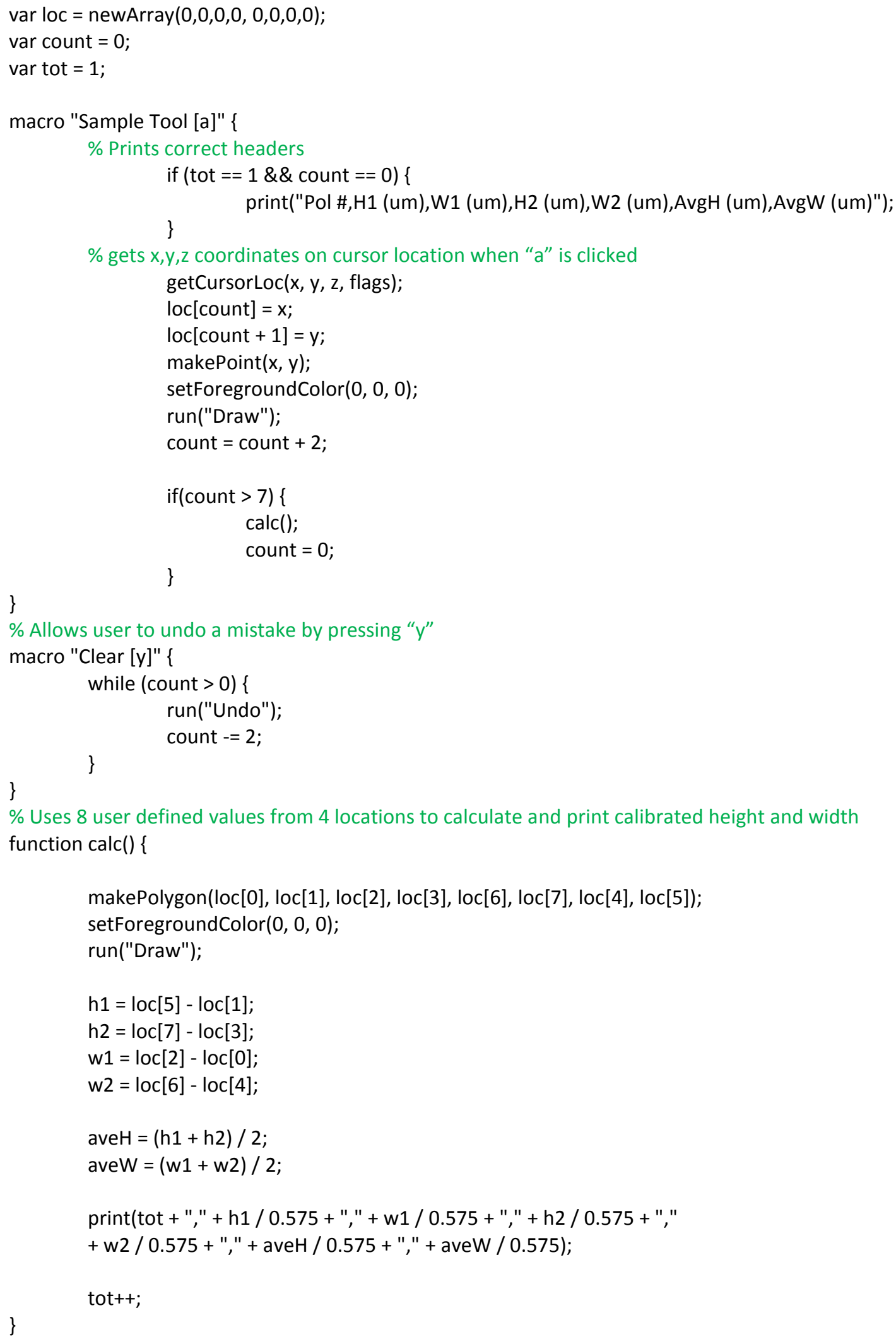




\section{Appendix D - How to use Automation Macro Protocol}

To use the tool, first move the automation tool file "automation tool.ijm" into Image J's macros folder, most likely found at the path seen in figure A on the following page. Next, run ImageJ and click to install a new macro (B). Find "automation tool.ijm" and open it. Make sure the "point tool" is selected on the Image J tool bar and define boxes around a vessel by clicking in the correct order (C). Once every vessel has been defined and measured, open the text log and save it (D). Next, open excel and choose to open a file; find the saved text log from ImageJ (if the text log isn't found change the file types in Open from "All Excel Files" to "All Files") (E). Upon arrival to the text import wizard; make sure "delimited" is checked on the first page (F) and that "tab" is checked on the second page $(G)$ and then click finish to get the results in an excel format $(H)$. 


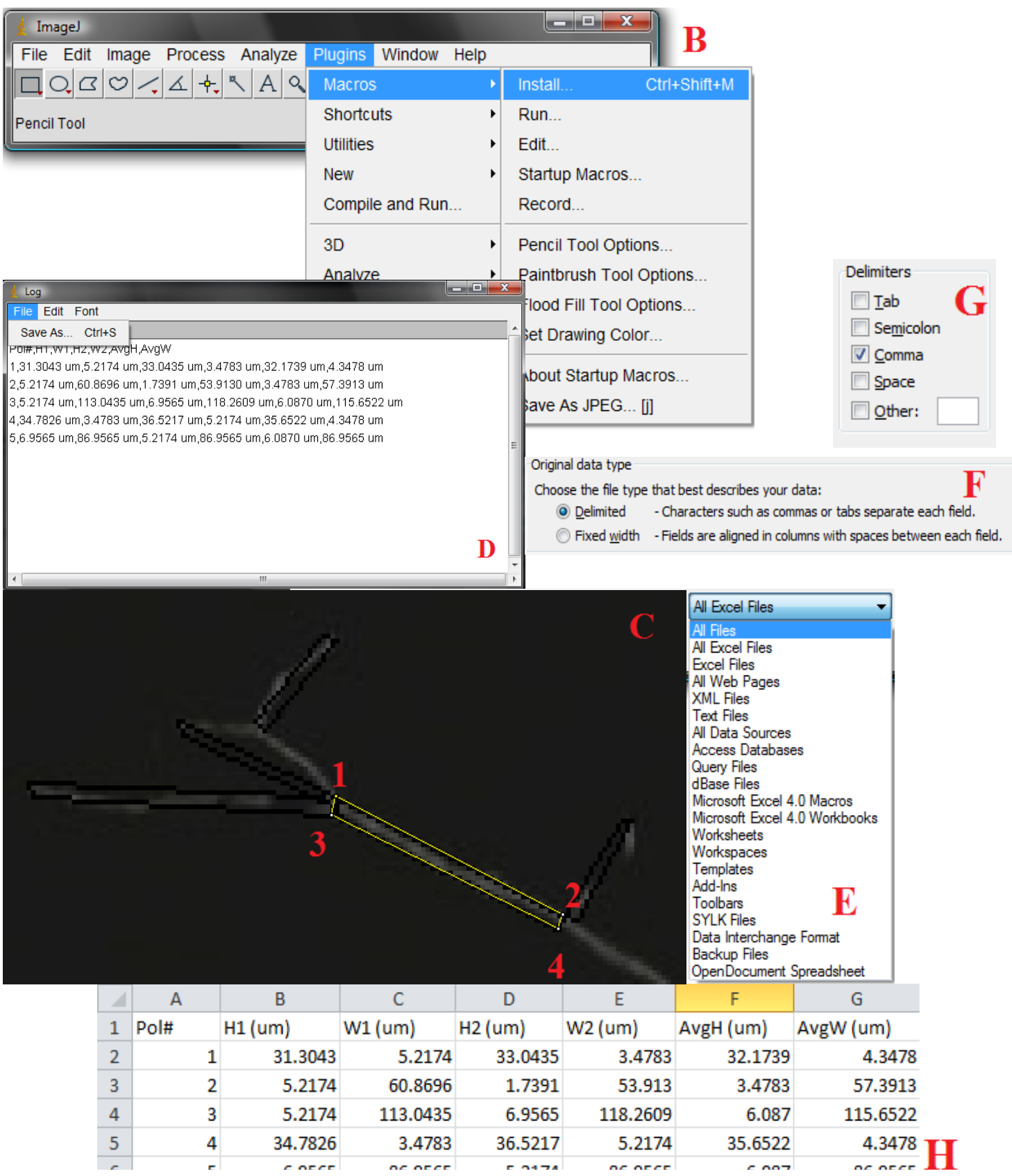

The steps to install and run the automation tool. A) The most common location of the ImageJ macros folder where the "automation tool.ijm" file should be copied. B) How to install a new macro. C) The correct click order to define a vessel for measurement. D) The log file of measurements that must be saved in order to export to excel. E) Select "all files" in order to open your log file in excel. F) Import screen 1 - make sure delimited is checked. G) Import screen 2 - make sure comma is checked. H) Example of final Excel output. 


\section{Appendix E - Macro versus Manual statistical comparison}

Repeated measures analysis setup in JMP (this window can be accessed in JMP by going to Analyze>Fit Model in the toolbar) and Tukey-Kramer results.

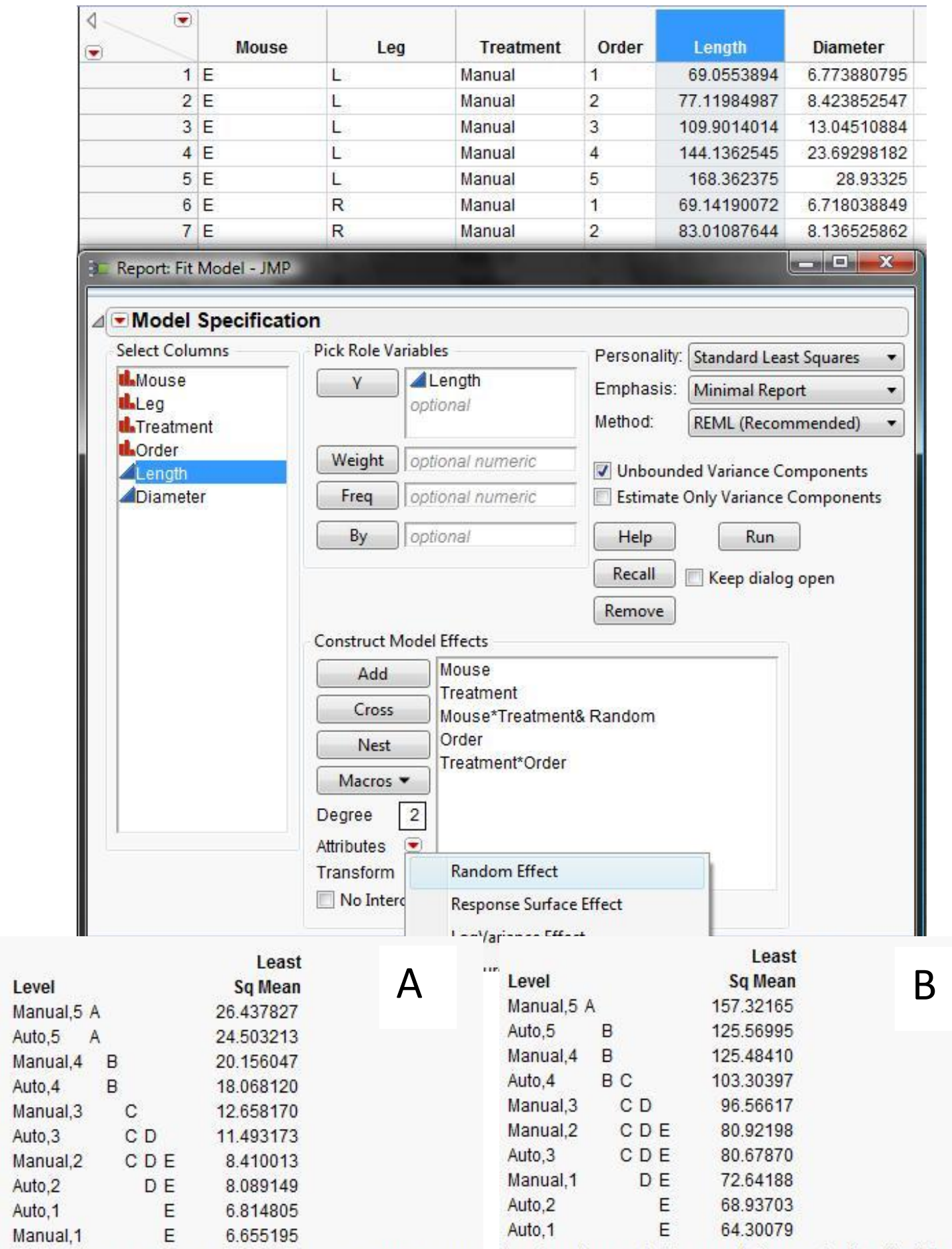

Levels not connected by same letter are significantly different.

Levels not connected by same letter are significantly different.

\section{Tukey-Kramer Results - A) Diameters B) Lengths}


Appendix F - Ligated Animal Vascular Casts (NI - Sham Surgery, I Ischemic/Ligated)
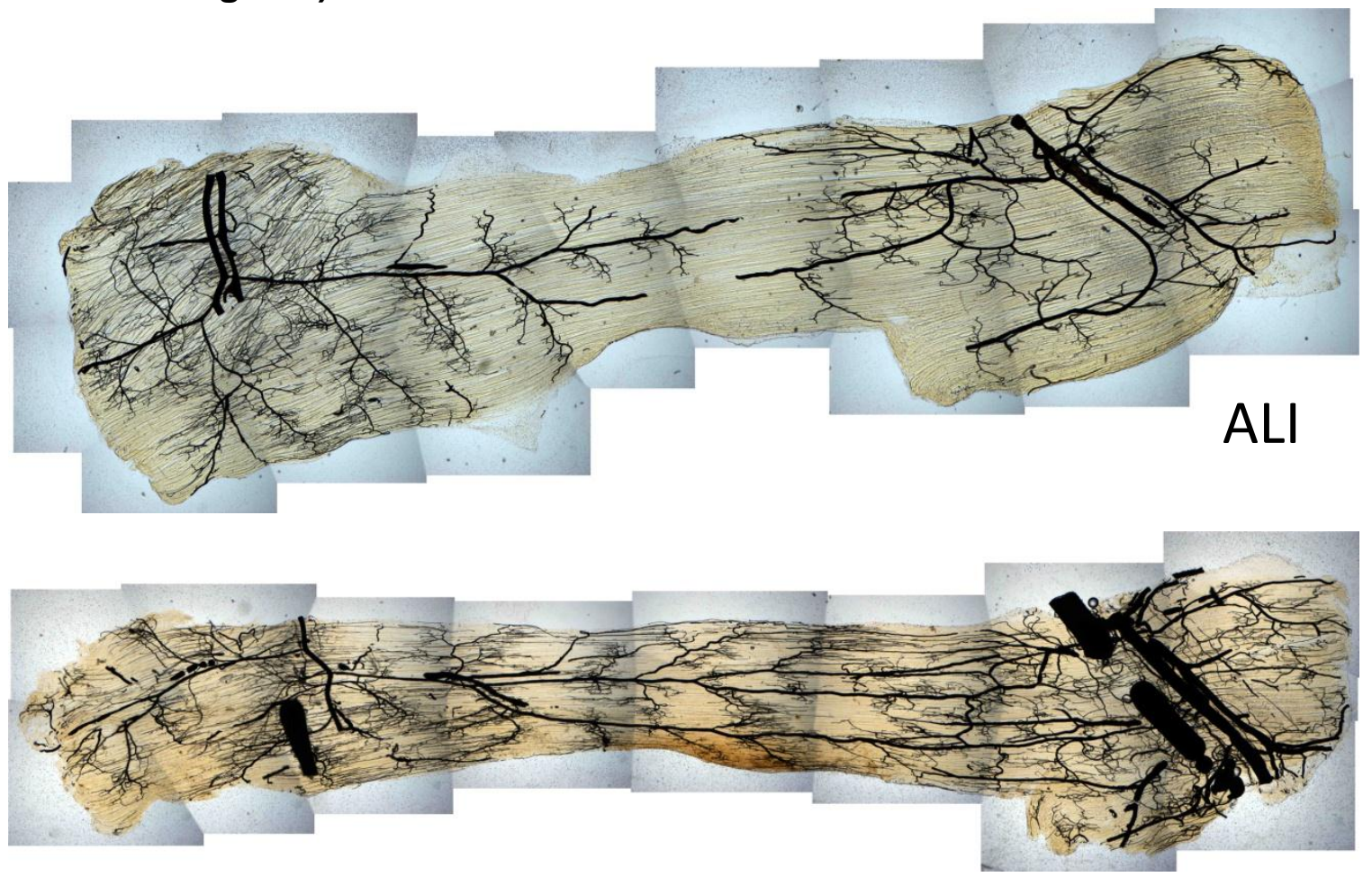

ARNI
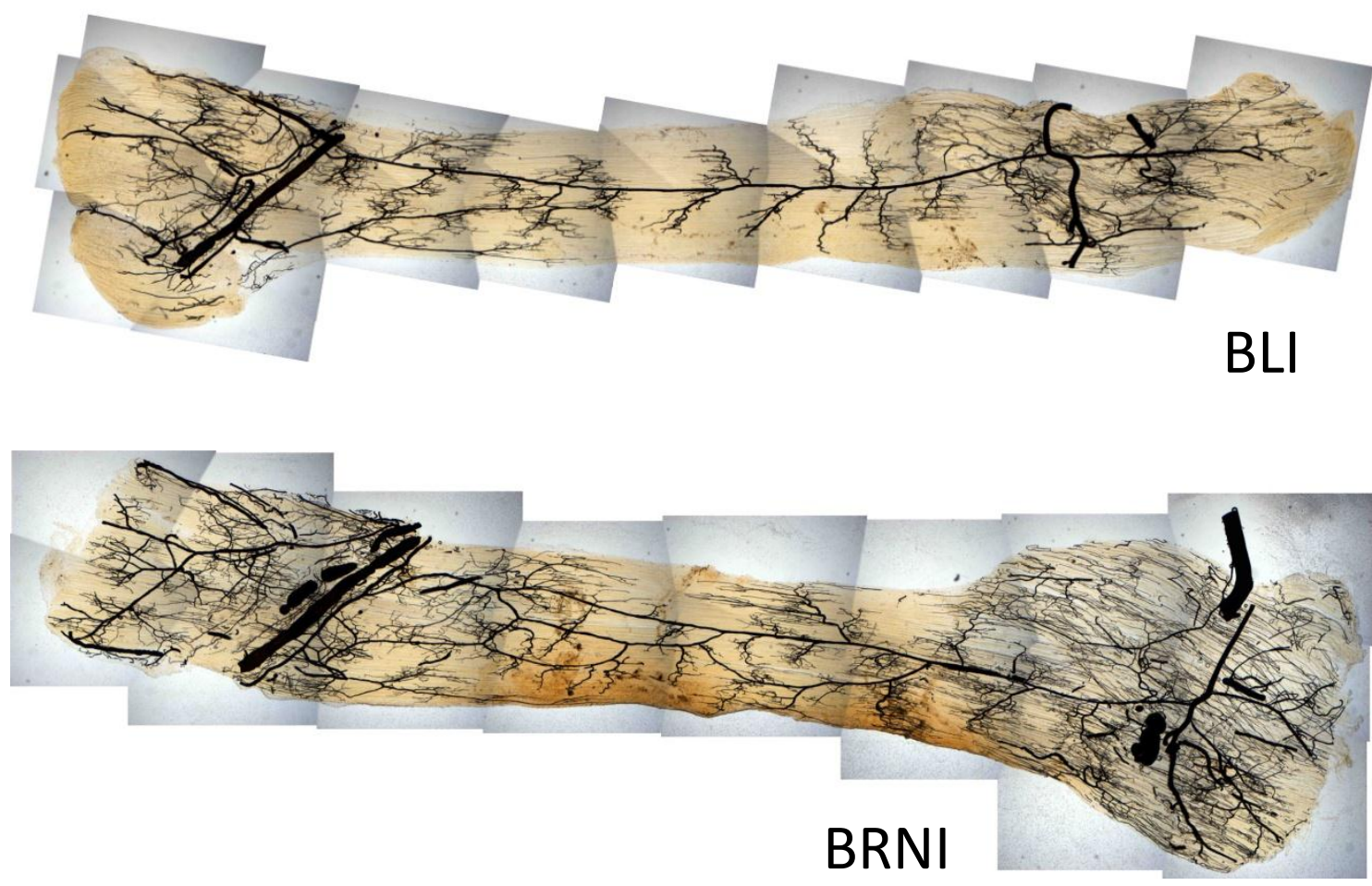


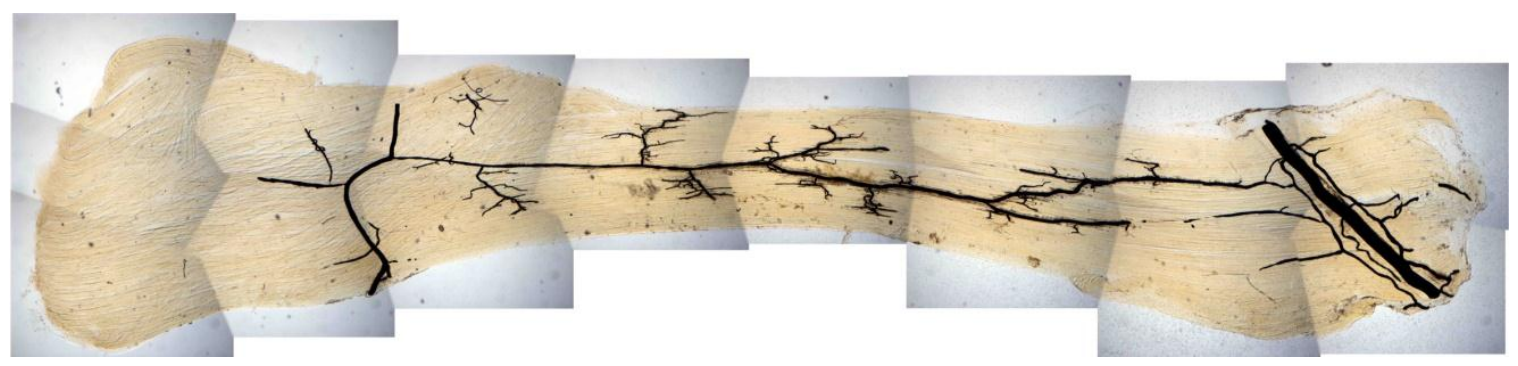

CLI

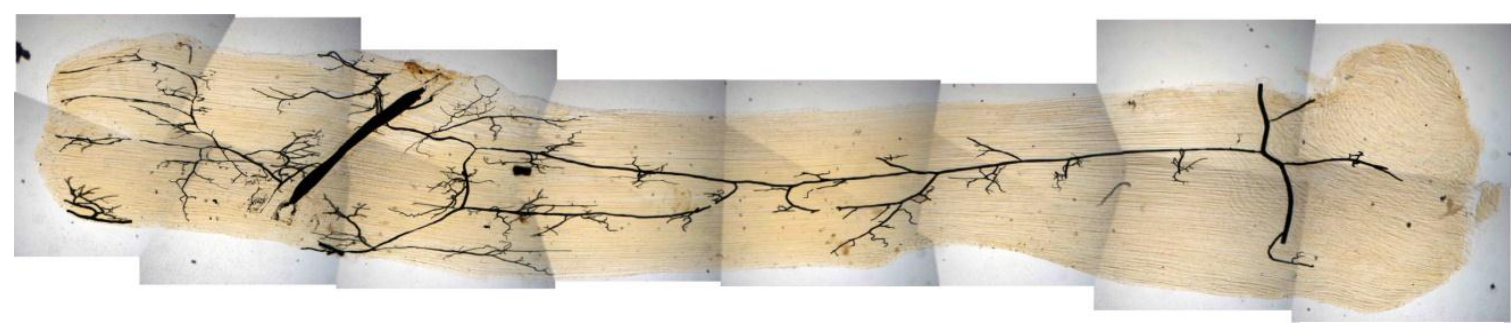

CRNI
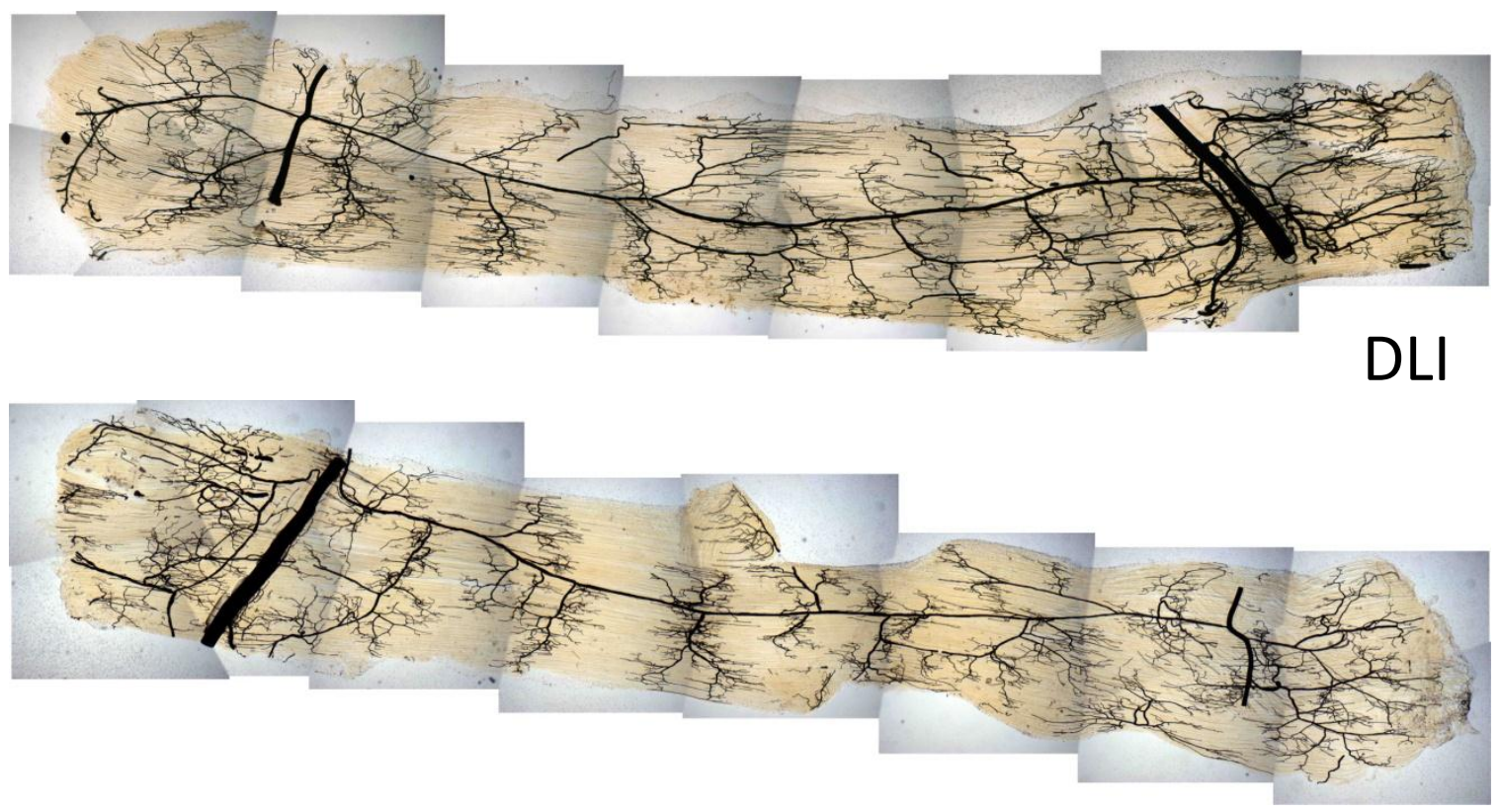

DRNI 

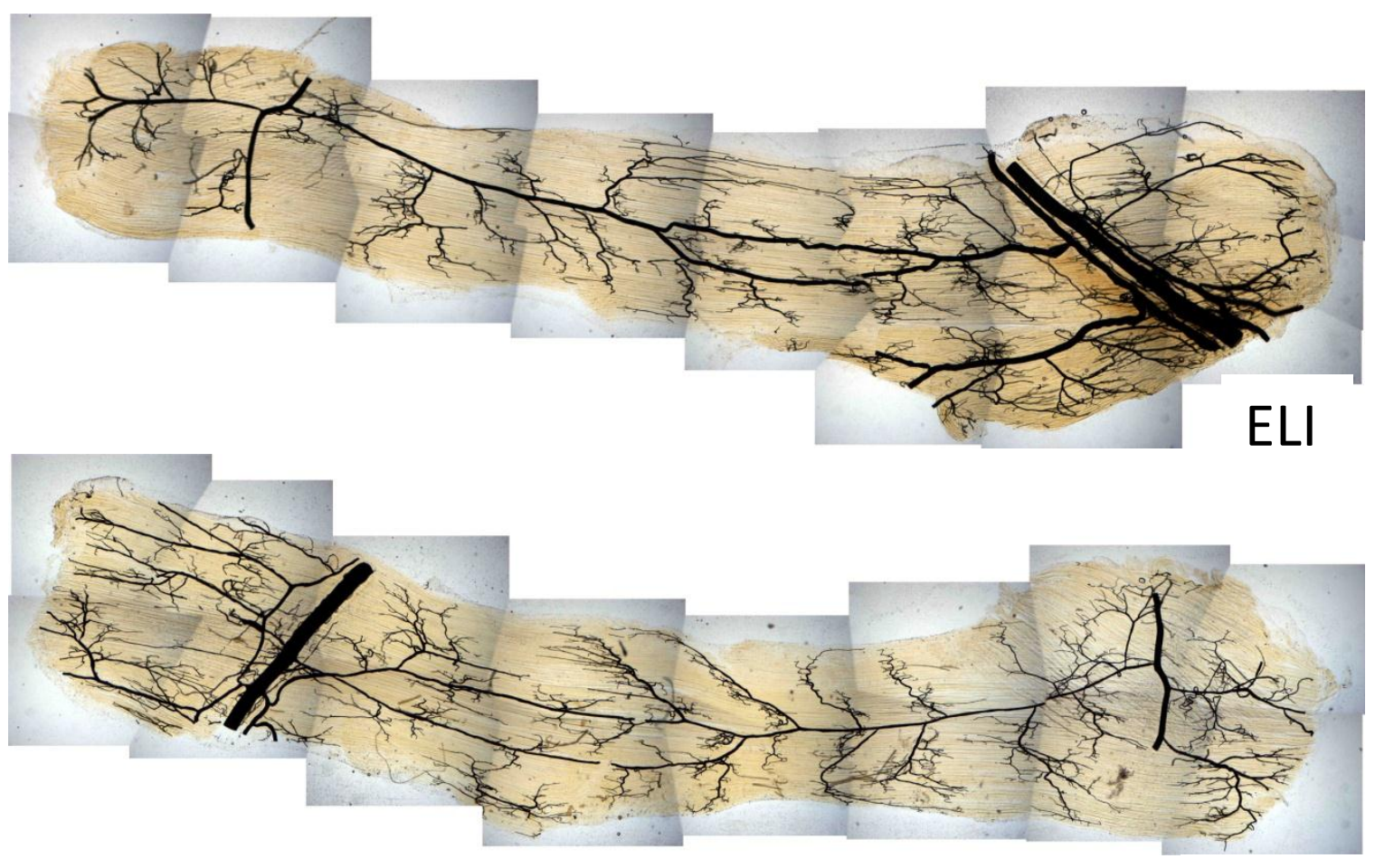

ERNI
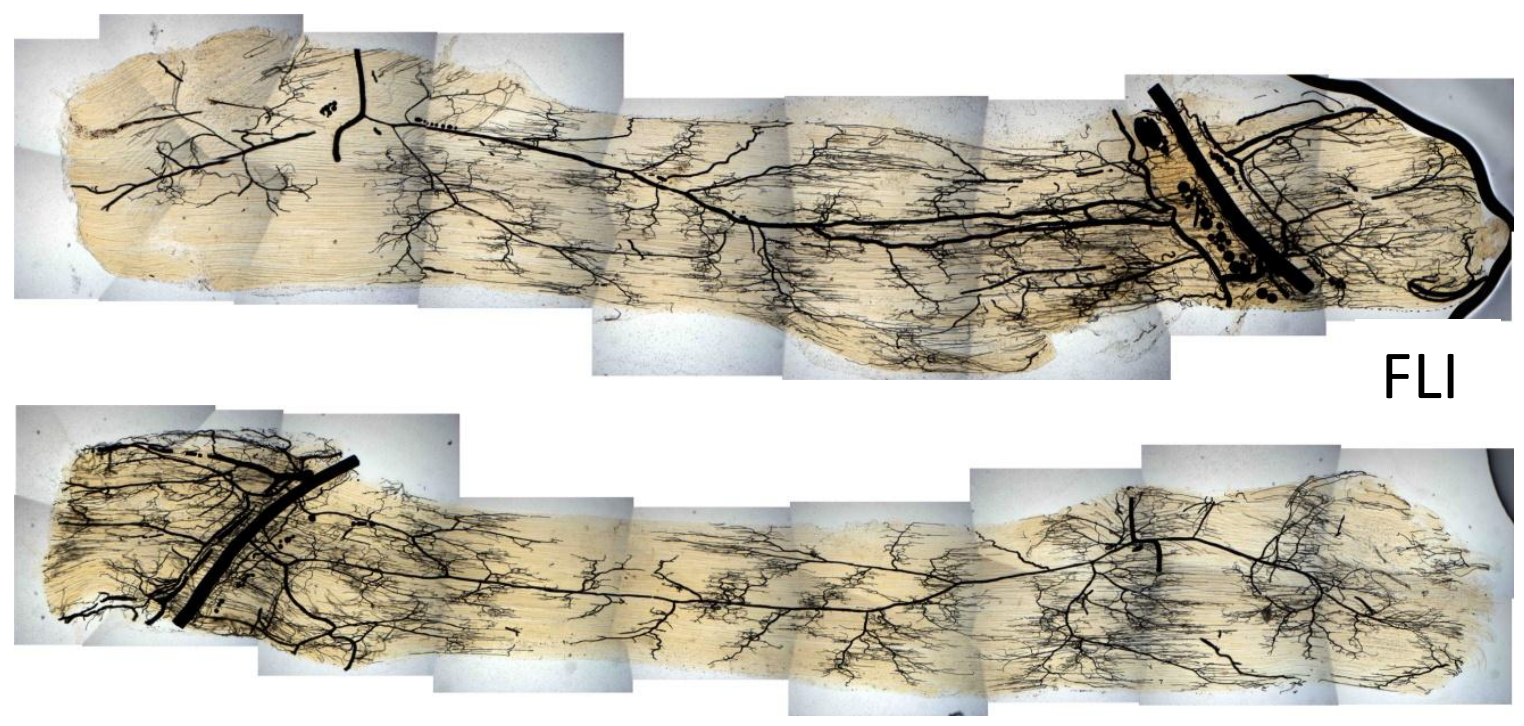

FRNI 

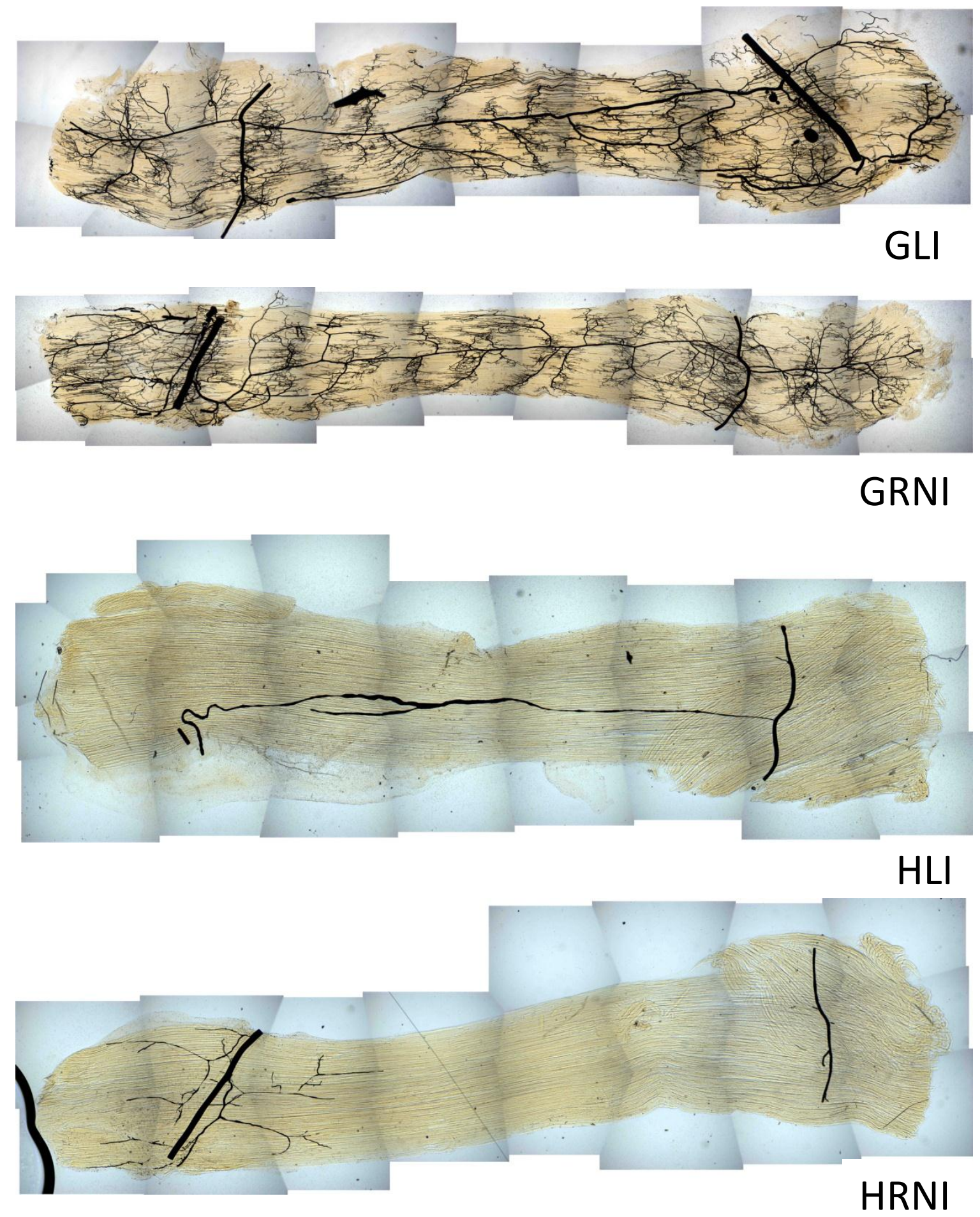


\section{Appendix G - Averages of samples. Order 6 diameters were taken from 5 measurements along the longitudinal arteriole, from the saphenous artery to the profunda femoris, which were then averaged. Order 6 length measurements were not taken.}

\begin{tabular}{|c|c|c|c|c|c|c|c|c|}
\hline & Order 1 & Averages & Order 2 & Averages & Order 3 & Averages & Order 4 & Averages \\
\hline & Length & Diameter & Length & Diameter & Length & Diameter & Length & Diameter \\
\hline ALI & 86.24144 & 10.48882 & 98.03744 & 11.15917 & 96.1163 & 17.31361 & 140.5446 & 23.19053 \\
\hline ARNI & 98.48015 & 9.264024 & 108.304 & 11.14781 & 133.4424 & 16.31226 & 164.4111 & 24.63949 \\
\hline BLI & 105.3227 & 5.881529 & 92.59553 & 7.14922 & 88.40336 & 12.03754 & 159.3638 & 19.80035 \\
\hline BRNI & 104.6846 & 7.538469 & 110.6882 & 11.31782 & 127.2148 & 14.46092 & 164.8611 & 23.61522 \\
\hline $\mathrm{CL}^{\sim}$ & * & * & * & * & * & * & * & * \\
\hline $\mathrm{CRNI}^{\sim}$ & * & * & * & * & * & * & * & * \\
\hline DLI & 103.3425 & 7.515439 & 100.2453 & 9.759459 & 115.5508 & 14.03512 & 147.8439 & 20.46152 \\
\hline DRNI & 84.8262 & 6.605741 & 88.46701 & 9.134799 & 108.9965 & 13.26662 & 136.9234 & 23.03898 \\
\hline & פרכב 24 & 1923057 & 230608 & 1173978 & 1600162 & & ברא & \\
\hline ERNI & 81.5188 & 8.367266 & 94.50524 & 11.17305 & 133.164 & 16.95995 & 157.6848 & 26.06367 \\
\hline & & & & & & & & \\
\hline FLI & 113.9675 & 7.871321 & 107.8098 & 10.16107 & 115.7072 & 13.68457 & 154.324 & 21.32366 \\
\hline FRNI & 81.7196 & 7.412631 & 76.82042 & 9.880871 & 100.207 & 15.058 & 129.9762 & 22.41798 \\
\hline & & & & & & & & \\
\hline GLI & 83.22666 & 7.488039 & 91.13299 & 10.8569 & 103.86 & 13.10716 & 130.368 & 21.76098 \\
\hline GRNI & 80.03319 & 7.161799 & 74.06476 & 9.130441 & 103.8021 & 13.06518 & 122.7058 & 20.59411 \\
\hline Нн & * & * & * & * & * & * & * & * \\
\hline HRNI $\sim$ & * & * & * & * & * & * & * & * \\
\hline & & & & & & & & \\
\hline & & & & & & & & \\
\hline $\mathrm{ACLNI}^{\sim}$ & * & * & * & * & * & * & * & * \\
\hline ACRNI ${ }^{\sim}$ & * & * & * & * & $*$ & * & $*$ & $*$ \\
\hline BCLNI & 74.40352 & 7.820062 & 82.73116 & 9.016035 & 113.8887 & 14.34068 & 143.2749 & 18.2212 \\
\hline BCRNI & 70.65889 & 7.236068 & 78.79303 & 9.179811 & 139.0158 & 14.70697 & 152.7827 & 20.97495 \\
\hline CCLNI $^{\sim}$ & 86.87762 & 9.621216 & 95.80622 & 14.15776 & 151.0991 & 18.40343 & 174.283 & 18.3284 \\
\hline CCRNI $^{\sim}$ & 87.13613 & 9.684577 & 102.6852 & 13.45771 & 132.131 & 18.64002 & 184.8781 & 23.70671 \\
\hline DCLNI & 83.80956 & 6.740432 & 89.74954 & 8.005522 & 104.2323 & 12.19378 & 147.9873 & 20.21939 \\
\hline DCRNI & 81.22603 & 6.697802 & 74.09918 & 9.347648 & 81.2258 & 14.08127 & 128.9058 & 22.36239 \\
\hline ECLNI & 69.05539 & 6.773881 & 77.11985 & 8.423853 & 109.9014 & 13.04511 & 144.1363 & 23.69298 \\
\hline ECLNI-Auto & 59.20069 & 7.302052 & 67.49971 & 8.122164 & 96.84117 & 11.44632 & 124.4901 & 21.13834 \\
\hline ECRNI & 69.1419 & 6.718039 & 83.01088 & 8.136526 & 92.96706 & 13.07546 & 134.3272 & 19.23071 \\
\hline ECRNI-Auto & 60.6356 & 6.101981 & 67.27136 & 8.350832 & 75.22914 & 11.97415 & 114.5164 & 15.0488 \\
\hline FCLNI & 75.49206 & 6.307358 & 84.78467 & 7.52497 & 94.99223 & 11.19354 & 120.4318 & 18.52443 \\
\hline FCLNI-Auto & 69.95467 & 6.944222 & 75.57411 & 7.377219 & 80.24982 & 10.76628 & 97.02094 & 16.79549 \\
\hline FCRNI & 76.87815 & 6.821504 & 78.77254 & 9.554703 & 88.404 & 13.31857 & 103.0412 & 19.17607 \\
\hline FCRNI-Auto & 67.41219 & 6.910965 & 65.40293 & 8.506379 & 70.39465 & 11.78595 & 77.18841 & 19.28985 \\
\hline GCLNI & 77.01696 & 7.072759 & 83.37722 & 9.182634 & 101.2262 & 14.63539 & 138.3154 & 21.6289 \\
\hline GCRNI & 84.89486 & 7.834427 & 90.78625 & 10.14882 & 102.2305 & 15.65571 & 127.1546 & 20.08568 \\
\hline HCLNI & 95.01164 & 7.815466 & 103.8533 & 11.84432 & 159.8453 & 17.76566 & 221.1134 & 25.06318 \\
\hline HCRNI ${ }^{\sim}$ & 89.49373 & 8.459142 & 106.5954 & 11.40382 & 132.2133 & 17.92901 & 201.8502 & 24.5897 \\
\hline
\end{tabular}




\begin{tabular}{|c|c|c|c|c|c|c|c|c|}
\hline & Order 5 & Averages & SA & 2 & 3 & 4 & BB & Average \\
\hline & Length & Diameter & & & & & & \\
\hline ALI & 142.4357 & 33.16433 & 66.087 & 56.473 & 53.993 & 43.494 & 45.217 & 53.0528 \\
\hline ARNI & 260.8131 & 30.3156 & 32.125 & 31.304 & 36.425 & 35.347 & 39.903 & 35.0208 \\
\hline BLI & 194.4492 & 27.78067 & 45.625 & 40.87 & 42.688 & 41.739 & 33.958 & 40.976 \\
\hline BRNI & 289.7884 & 27.13387 & 43.283 & 34.036 & 29.89 & 54.517 & 35.205 & 39.3862 \\
\hline $\mathrm{CLI}^{\sim}$ & * & * & 41.739 & 56.279 & 45.782 & 35.604 & 24.348 & 40.7504 \\
\hline $\mathrm{CRNI}^{\sim}$ & $*$ & * & 24.348 & 34.9 & 33.011 & 27.826 & 25.169 & 29.0508 \\
\hline DLI & 302.904 & 26.05036 & 55.189 & 45.515 & 48.666 & 40.821 & 27.254 & 43.489 \\
\hline DRNI & 212.1664 & 28.42638 & 33.768 & 38.132 & 38.261 & 24.348 & 32.125 & 33.3268 \\
\hline ELI & 274.4226 & 33.8706 & 65.579 & 64.123 & 68.639 & 54.437 & 51.972 & 60.95 \\
\hline ERNI & 191.0467 & 31.07833 & 38.695 & 33.865 & 29.565 & 32.125 & 31.207 & 33.0914 \\
\hline FLI & 204.4563 & 30.16407 & 55.671 & 51.507 & 63.234 & 45.472 & 46.077 & 52.3922 \\
\hline FRNI & 315.4578 & 23.293 & 26.908 & 33.986 & 30.419 & 33.189 & 28.929 & 30.6862 \\
\hline GLI & 181.1062 & 27.996 & 29.855 & 30 & 29 & 20.963 & 17.236 & 25.4108 \\
\hline GRNI & 155.7559 & 26.01423 & 29.153 & 31.497 & 41.017 & 31.891 & 25.589 & 31.8294 \\
\hline HLI $\sim$ & * & * & 36.24 & 46.435 & 65.761 & 91.734 & 48.433 & 57.7206 \\
\hline HRNI ${ }^{\sim}$ & $*$ & * & * & * & * & * & $*$ & \\
\hline $\mathrm{ACLNI}^{\sim}$ & * & * & 18.282 & 16.726 & 24.051 & 17.586 & 12.205 & 17.77 \\
\hline ACRNI $^{\sim}$ & $*$ & * & 28.518 & 24.375 & 16.397 & 19.744 & 22.029 & 22.2126 \\
\hline BCLNI & 117.4022 & 24.96456 & 34.956 & 22.392 & 20.95 & 24.334 & 18.332 & 24.1928 \\
\hline BCRNI & 117.3414 & 26.21171 & 36.314 & 17.413 & 20.888 & 23.623 & 20 & 23.6476 \\
\hline $\mathrm{CCLNI}^{\sim}$ & * & $*$ & 24.928 & 22.616 & 24.021 & 24.458 & 20.323 & 23.2692 \\
\hline CCRNI $^{\sim}$ & * & * & 34.281 & 28.881 & 19.829 & 17.401 & 19.964 & 24.0712 \\
\hline DCLNI & 167.1221 & 31.43587 & 30.022 & 32.977 & 35.984 & 29.571 & 27.419 & 31.1946 \\
\hline DCRNI & 132.6518 & 29.8144 & 40.833 & 30.744 & 31.413 & 29.769 & 24.595 & 31.4708 \\
\hline ECLNI & 168.3624 & 28.93325 & 30.373 & 30.417 & 31.905 & 30.774 & 30.593 & 30.8124 \\
\hline ECLNI-Auto & 110.2174 & 28.58698 & $x$ & $x$ & $x$ & $x$ & $x$ & \\
\hline ECRNI & 168.5718 & 23.083 & 31.926 & 26.971 & 32.361 & 21.809 & 32.361 & 29.0856 \\
\hline ECRNI-Auto & 138.5507 & 20.14493 & $x$ & $x$ & $x$ & $x$ & $x$ & \\
\hline FCLNI & 143.8183 & 26.88875 & 29.527 & 28.164 & 25.217 & 23.494 & 27.635 & 26.8074 \\
\hline FCLNI-Auto & 121.7391 & 25.86958 & $x$ & $x$ & $x$ & $x$ & $\mathrm{x}$ & \\
\hline FCRNI & 148.5342 & 26.84631 & 27.467 & 33.226 & 33.089 & 32.484 & 29.331 & 31.1194 \\
\hline FCRNI-Auto & 131.7726 & 23.41137 & $x$ & $x$ & $x$ & $x$ & $x$ & \\
\hline GCLNI & 120.9407 & 24.41 & 42.064 & 38.507 & 28.748 & 30.879 & 30.285 & 34.0966 \\
\hline GCRNI & 136.598 & 33.20075 & 55.223 & 43.695 & 32.268 & 38.261 & 31.316 & 40.1526 \\
\hline HCLNI & 193.6465 & 18.6795 & 41.339 & 34.826 & 31.22 & 33.055 & 22.575 & 32.603 \\
\hline HCRNI ${ }^{\sim}$ & * & * & 27.826 & 16.901 & 27.32 & 26.292 & 27.253 & 25.1184 \\
\hline
\end{tabular}




\section{Appendix H - Total Vessel Counts}

\begin{tabular}{|c|c|c|c|c|c|}
\hline & Order 1 & Order 2 & Order 3 & Order 4 & Order 5 \\
\hline ALI & 280 & 195 & 76 & 17 & 3 \\
\hline ARNI & 288 & 148 & 74 & 43 & 10 \\
\hline BLI & 376 & 173 & 74 & 23 & 6 \\
\hline BRNI & 292 & 201 & 90 & 36 & 15 \\
\hline $\mathrm{CLI}{ }^{\sim}$ & $*$ & $*$ & $*$ & * & * \\
\hline $\mathrm{CRNI}^{\sim}$ & $*$ & $*$ & * & * & * \\
\hline DLI & 581 & 292 & 146 & 69 & 14 \\
\hline DRNI & 499 & 289 & 133 & 58 & 8 \\
\hline ELI & 286 & 159 & 67 & 19 & 5 \\
\hline ERNI & 623 & 317 & 125 & 49 & 3 \\
\hline FLI & 549 & 303 & 129 & 56 & 15 \\
\hline FRNI & 567 & 341 & 146 & 52 & 6 \\
\hline GLI & 487 & 268 & 114 & 44 & 18 \\
\hline GRNI & 783 & 417 & 197 & 75 & 13 \\
\hline HLI $\sim$ & $*$ & $*$ & * & * & * \\
\hline $\mathrm{HRNI}^{\sim}$ & $*$ & * & * & * & * \\
\hline ACLNI $\sim$ & $*$ & $*$ & * & * & * \\
\hline ACRNI $\sim$ & $*$ & * & * & * & * \\
\hline BCLNI & 698 & 318 & 135 & 35 & 9 \\
\hline BCRNI & 575 & 280 & 116 & 38 & 7 \\
\hline CCLNI & 201 & 113 & 42 & 5 & * \\
\hline $\mathrm{CCRNI}^{\sim}$ & 241 & 119 & 52 & 7 & * \\
\hline DCLNI & 711 & 337 & 147 & 54 & 15 \\
\hline DCRNI & 516 & 287 & 104 & 51 & 5 \\
\hline ECLNI & 755 & 373 & 147 & 55 & 8 \\
\hline ECLNI-Aut & $x$ & $x$ & $x$ & $x$ & $x$ \\
\hline ECRNI & 695 & 348 & 148 & 49 & 6 \\
\hline ECRNI-Aut & $x$ & $x$ & $x$ & $x$ & $x$ \\
\hline FCLNI & 777 & 401 & 181 & 54 & 8 \\
\hline FCLNI-Aut & $x$ & $x$ & $x$ & $x$ & $x$ \\
\hline FCRNI & 916 & 464 & 195 & 60 & 13 \\
\hline FCRNI-Aut & $x$ & $x$ & $x$ & $x$ & $x$ \\
\hline GCLNI & 439 & 224 & 83 & 31 & 6 \\
\hline GCRNI & 485 & 232 & 95 & 28 & 8 \\
\hline HCLNI & 354 & 191 & 79 & 22 & 2 \\
\hline HCRNI $\sim$ & 353 & 191 & 67 & 10 & * \\
\hline Average & 544.8696 & 285.1304 & 121.7826 & 44.26087 & 8.826087 \\
\hline
\end{tabular}




\section{Appendix I - JMP output of Tukey-Kramer results for sham versus control and sham versus ligated}

$\begin{array}{lrrr}\text { Level } & & & \begin{array}{r}\text { Least } \\ \text { Sq Mean } \\ 237.50472\end{array} \\ \text { Sham,5 } & \text { A } & & 146.09374 \\ \text { Sham,4 } & \text { B } & 140.73944 \\ \text { Control,5 } & \text { B C } & 129.24232 \\ \text { Control,4 } & \text { B C D } & 117.80446 \\ \text { Sham,3 } & \text { B C D E } & 100.76863 \\ \text { Control,3 } & \text { B C D E } & 92.14160 \\ \text { Sham,2 } & \text { C D E } & 98.54376 \\ \text { Sham,1 } & \text { D E } & 81.09237 \\ \text { Control,2 } & \text { D E } & \text { E } \\ \text { Control,1 } & \text { E } & 76.55996 \\ \text { Levels not connected by same letter are significantly different. }\end{array}$

Sham versus control - Length

$\begin{array}{lrrr}\text { Level } & & \begin{array}{r}\text { Least } \\ \text { Sq Mean } \\ \text { Sham,5 A }\end{array} & 237.50472 \\ \text { Ligated,5 A } & & 216.62899 \\ \text { Sham,4 } & \text { B } & 146.09374 \\ \text { Ligated,4 } & \text { B C } & 142.52843 \\ \text { Sham,3 } & \text { B C } & 117.80446 \\ \text { Ligated,3 } & \text { B C } & 113.27565 \\ \text { Ligated,2 } & \text { B C } & 102.29698 \\ \text { Ligated,1 } & \text { B C } & 97.74053 \\ \text { Sham,2 } & \text { C } & 92.14160 \\ \text { Sham,1 } & \text { C } & 88.54376\end{array}$

Levels not connected by same letter are significantly different. Sham versus ligated - Length

$\begin{array}{lllr}\text { Level } & & & \begin{array}{r}\text { Least } \\ \text { Sq Mean } \\ \text { Sham,6 A }\end{array} \\ \text { Control,6 A B } & & 33.198800 \\ \text { Sham,5 } & \text { B } & & 28.359775 \\ \text { Control,5 } & \text { B } & & 27.338944 \\ \text { Sham,4 } & \text { B C } & & 23.362135 \\ \text { Control,4 } & \text { C D } & 18.896860 \\ \text { Sham,3 } & \text { D E } & 14.482530 \\ \text { Control,3 } & \text { E F } & 12.698495 \\ \text { Sham,2 } & \text { F G } & 9.926175 \\ \text { Control,2 } & & \text { G } & 7.804402 \\ \text { Sham,1 } & \text { F G } & 7.353698 \\ \text { Control,1 } & \text { G } & 5.592468\end{array}$

Levels not connected by same letter are significantly different.

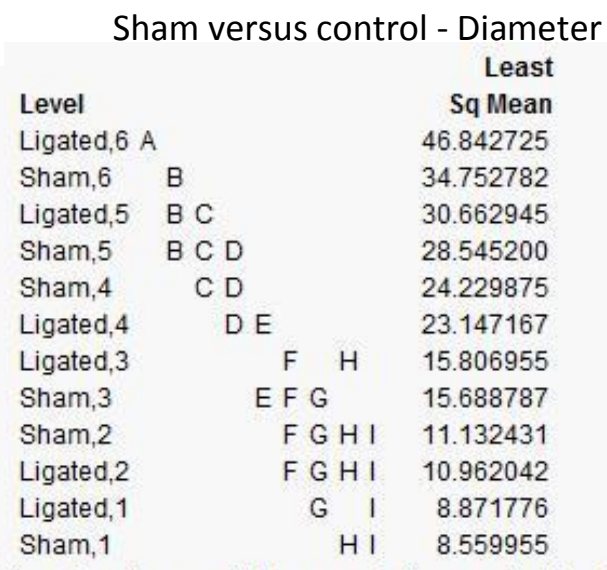

Levels not connected by same letter are significantly different. Sham versus ligated - Diameter 


\section{Appendix J - Statistical Setup in JMP for sham versus control and sham versus ligated}

The screen shots on the following page show how to set up a repeated measures analysis in JMP. In the JMP home window access this screen by going to Analyze>Fit Model. In the screen shots, behind the fit model window shows how the data was organized. The $Y$ variable is what is being measured; for this analysis it was either vessel length or diameter. The construct model effects window is where the different treatments, experimental units and effects are placed for the correct analysis. A) The model fit for the sham versus control length analysis. The treatment (sham or control) is nested within the mouse variable because treatments were delivered across different mice. B) The model fit for the sham versus ligated length analysis. Blocking on the mouse was done to remove variability within the experiment because the treatment (sham or ligation) was done on the same mouse. This is why the added "Mouse" variable is seen in the construct model effects window as well as mouse being crossed with treatment instead of being nested as before. 

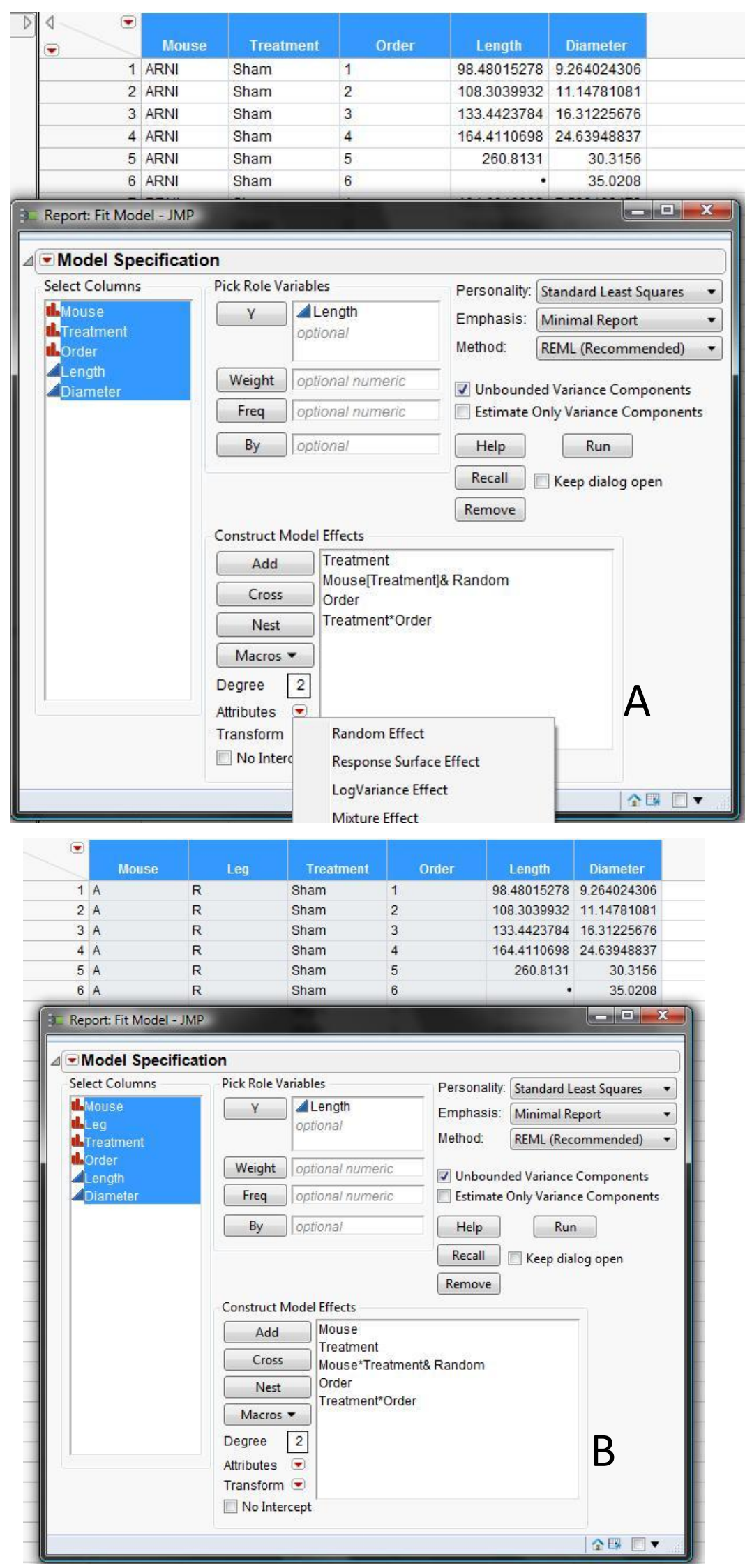


\section{Appendix K - Femoral Artery Ligation Checklist}

Date __ Hindlimb Ischemia Surgery - Ligation Initials

Mouse Information

DOB:

Sex:

Tag:

Genotype/strain

Cage:

35. Spray right hindlimb with Nolvasan

36. Return animal to anes thesia box

37. Apply $4 \times 4$ gauze sponge to heat pad to protect animal from exces sive heat

38. Affix non-rebreathing circuit to surgery table w/ chemistry clamp

39. Lay animal supine on circulating heat pad $\mathrm{w} /$ nose in nose-cone

Materials

Sterilize-autoclave or flash autoclave

Standard pattern forceps (1)

S\&T forceps (2)

$5-45$ forceps (1)

Iris scissors (1)

Microdissection scissors (1)

Castroviejo (1)

Retractor (1)

Pre-sterilize in autoclave

8. cotton gauze (2)

9. cotton 5 wabs (12)

10. 6.0 sillk suture $(2 \times 1$-inch $)$

Obtained in surgery suite

11. sterile Petri dish w/sterile saline

sterile gloves

sterile 7.0 prolene suture

14. heat-cautery
15 . FST heat pad w/ rectal probe

16. heat pad

17. recovery bin \& weigh boat

18. depilatory cream

19. non-sterile cotton swabs

20. non-sterile cotton gauze

21. isolation mask \& cap

22. analgesic (Buprenorphine)

Surgery preparation

23. Spray surgery area with Nolvas an

24. Weigh animal in weight boat

25. Place animal in anes thesia box

26. Open the oxygen cylinder and set anes thesia-machine flow meter to $\sim 31 \cdot \mathrm{min}^{-1}$

27. Anesthetize animal $w / 5 \%$ is oflurane

28. Give the animal an subcutaneous injection of buprenorphine $(0.075 \mathrm{mg} / \mathrm{kg})$

29. Affix non-rebreathing circuit to bench-top with tape

30. Reduce flow rate to $0.5-1.01 \cdot \mathrm{min}^{-1}$ and the is oflurane to $1-3 \%$

31. Apply ear tag high on left ear

32. Lay animal supine with nose in nose-cone

33. Shave hair on the right hindlimb \& lower abdomen with clippers

34. Remove excess hair with depilatory cream

40. Insert rectal probe and set themo-controller to $37^{\circ} \mathrm{C}$

41. Apply veterinary ointment to eyes to avoid drying during procedure

42. Apply veterinary ointment to anus and place rectal probe $\sim 1 \mathrm{~cm}$ into anus to monitor corebody temperature

Surgery

43. Make a small incision on the middle, medial aspect of the left thigh

44. Extend the incision up to the abdominal wall

45. Blunt dis sect the subcutaneous connective tis sue to maximize surgical exposure

46. Use cautery to remove fat pad overlying femoral a-v pair \& cauterize epigastric avpair

47. Blunt dissect the femoral artery from the neurovas cular bundle just downstream from the deep femoral branch

48. Tie off the femoral artery \& vein with 6.0 sillk suture, just downstream to the deep femoral branch

49. Use 6.0 polypropylene suture to clo se the skin

50. Make a small incision on the middle, medial aspect of the right thing

51. Extend the incision up to the abdominal wall

52. Blunt dis sect the subcutaneous connective tis sue to maximize surgical exposure

53. Use 6.0 polypropylene suture to clo se the skin

Post-Surgical

54. Place the animal in the recovery bin, on a blue bench cover, above a heat pad and allow to recover

55. Turn flow meter down to 0 , turn off is oflurane, and clo se the oxygen cylinder

56. Indicate surgery on cage card

Notes 


\section{Appendix L - Manual Measurement Protocol}

1) Calibrate ImageJ (see figure A below)

2) Select the freehand lines tool (see figure B below) to trace the vessel segments that need measuring.

3) Press $<\mathrm{Ctrl}+\mathrm{M}>$ to measure the segment length. Note: Vessel length and diameter need to be measured separately, and it's easiest to measure a vessels length, followed by the diameter, as opposed to measuring all the lengths and then all the diameters, which makes it difficult to organize in the results window. Measurements can then be copied into Excel to be analyzed.

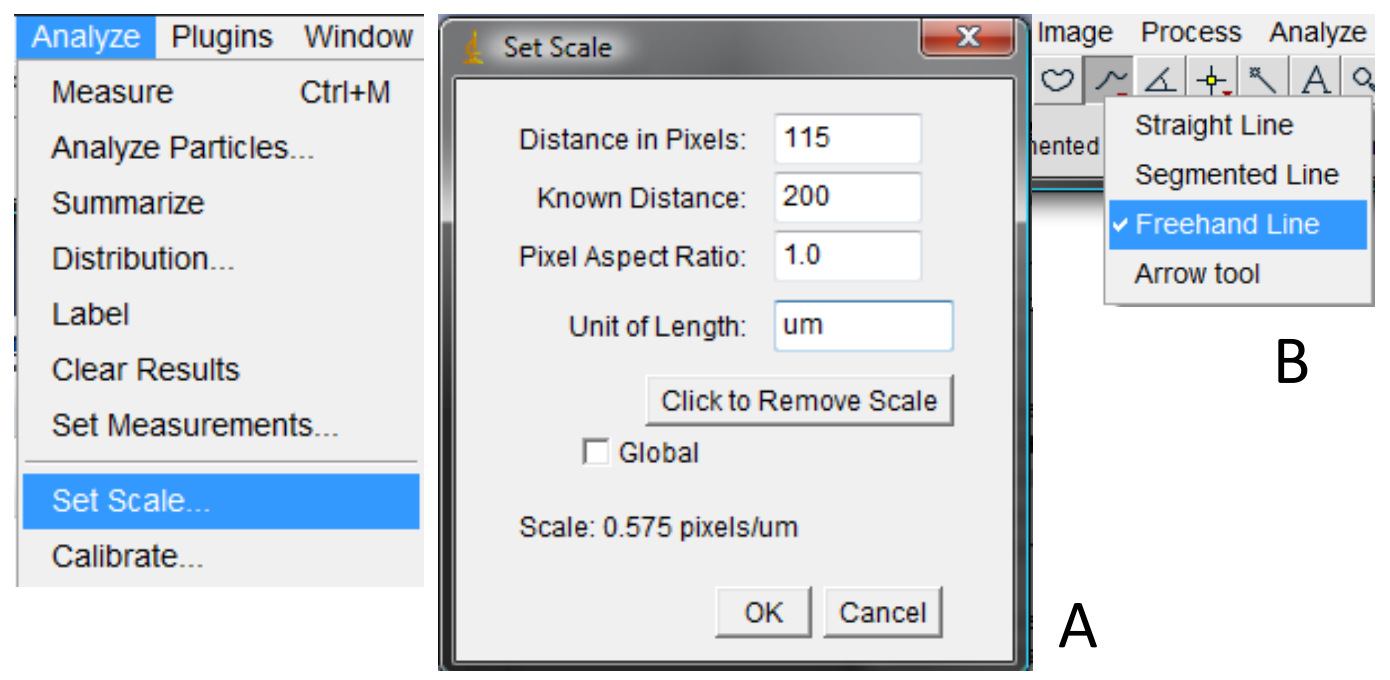

Steps to measure an image manually in ImageJ. A) How to set the proper scale. B) How to select the freehand line for tracing a vessel. 


\section{Appendix M - Photoshop CS3 Photomerge Protocol}

First, open all the files to photomerge, select the photomerge function from the automate menu. Next, select all the files to be photomerged. Photomerge will attempt to automatically arrange all the images into a single panorama. Because of the intricacies of the vessels, photomerge sometimes fails at automatically arranging the images. In this instance, manual arrangement is necessary. As a check I would name my image files in order to make sure Photoshop arranged them properly and for ease of manual arrangement.

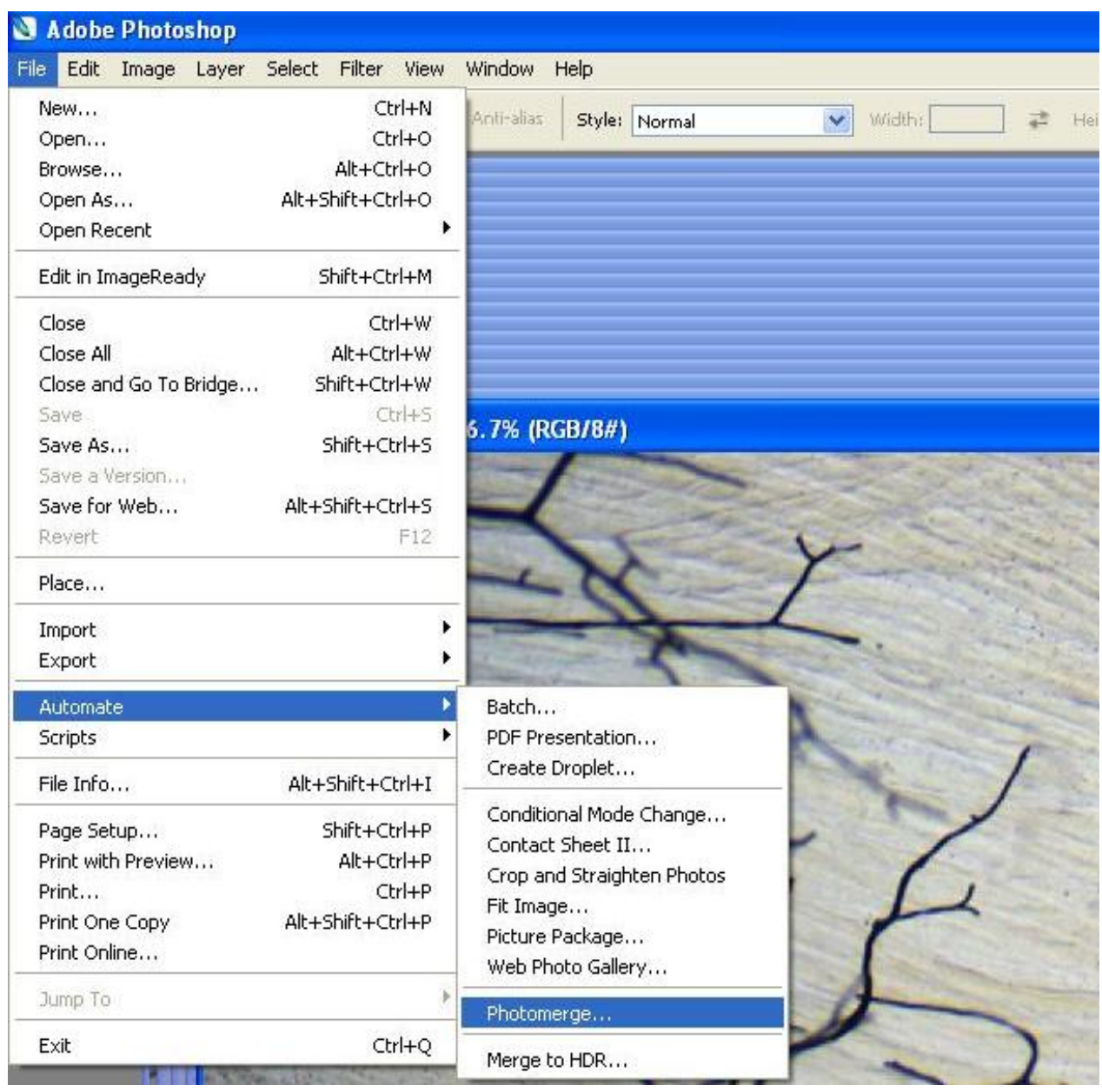




\section{Appendix N - T-test statistical results for vessel count}

\begin{tabular}{|c|c|c|c|c|c|c|c|c|c|c|}
\hline & Order 5 & & Order 4 & & Order 3 & & Order 2 & & Order 1 & \\
\hline & Ligated & Sham & Ligated & Sham & Ligated & Sham & Ligated & Sham & Ligated & Sham \\
\hline A & 3 & 10 & 17 & 43 & 76 & 74 & 195 & 148 & 280 & 288 \\
\hline B & 6 & 15 & 23 & 36 & 74 & 90 & 173 & 201 & 376 & 292 \\
\hline D & 14 & 8 & 69 & 58 & 146 & 133 & 292 & 289 & 581 & 499 \\
\hline$E$ & 5 & 3 & 19 & 49 & 67 & 125 & 159 & 317 & 286 & 623 \\
\hline $\mathrm{F}$ & 15 & 6 & 56 & 52 & 129 & 146 & 303 & 341 & 549 & 567 \\
\hline G & 18 & 13 & 44 & 75 & 114 & 197 & 268 & 417 & 487 & 783 \\
\hline Mean & 10.16666667 & 9.166667 & 38 & 52.16667 & 101 & 127.5 & 231.6666667 & 285.5 & 426.5 & 508.6667 \\
\hline \multirow[t]{2}{*}{ Std Error } & 2.54842 & 1.81506 & 8.84685 & 5.51009 & 13.52528 & 17.77404 & 25.89423 & 39.75907 & 53.6313 & 79.04963 \\
\hline & $p=0.75583$ & & $p=0.20393$ & & $p=0.26286$ & & $p=0.28302$ & & $p=0.40985$ & \\
\hline
\end{tabular}




\section{Appendix 0 - Image Permissions}

Figure 4 permissions

\section{Copyright}

Clearance

Center

\section{Springer}

Title:

New insights into intussusceptive angiogenesis

the language of science Author:

Publication: Springer eBook

Publisher: Springer

Date: Jan 1, 2005

Copyright @ 2005, Birkhäuser Basel

\section{Order Completed}

Thank you very much for your order.

This is a License Agreement between David Danzeiser ("You") and Springer ("Springer"). The license consists of your order details, the terms and conditions provided by Springer, and the payment terms and conditions.

\section{Get the printable license.}

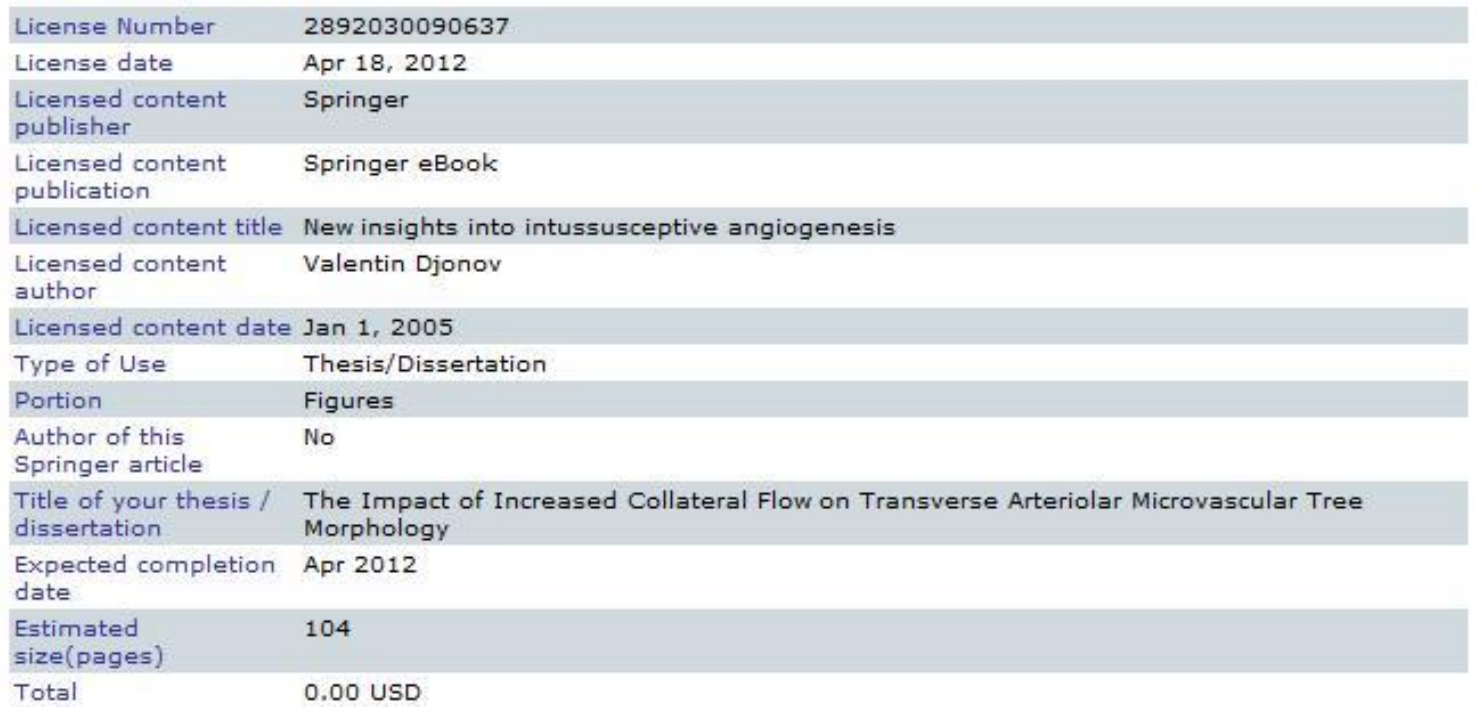


Figure 5 permissions

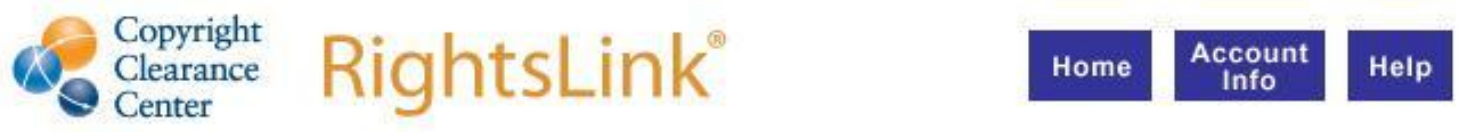
(3)WILEY ${ }^{\text {Tule }}$

Microvascular Remodeling: A Complex Continuum Spanning

Author: Angiogenesis to Arteriogenesis SKALAK SHAYN M. PEIRCE,THOMAS C. Logged in as: David Danzeiser

Publication: Microcirculation

Publisher: John Wiley and Sons

Date: Jan 26, 2010

2003 Blackwell

\section{Order Completed}

Thank you very much for your order.

This is a License Agreement between David Danzeiser ("You") and John Wiley and Sons ("John Wiley and Sons"). The license consists of your order details, the terms and conditions provided by John Wiley and Sons, and the payment terms and conditions.

Get the printable license.

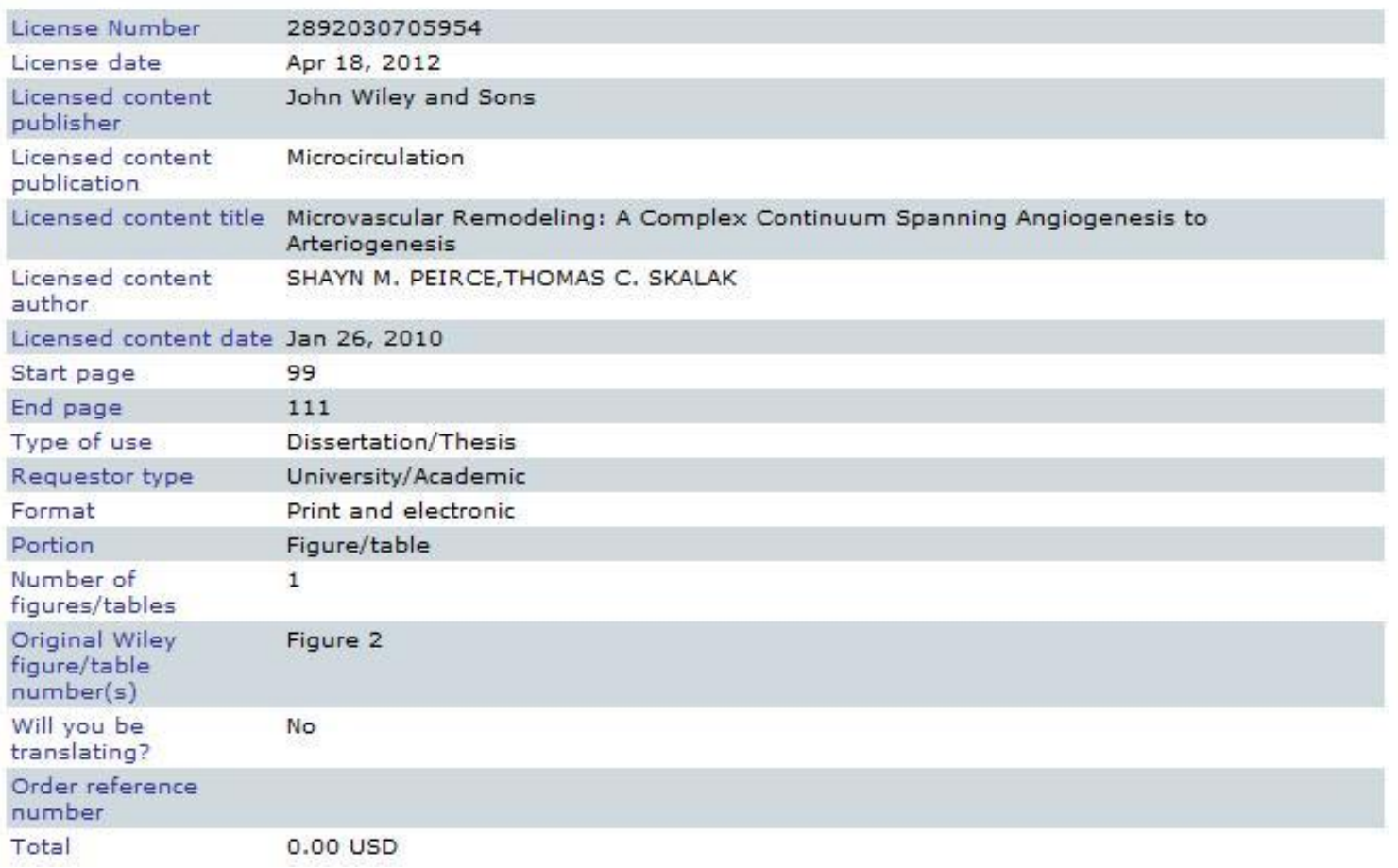

